\title{
Late Hallstatt Female Head/Hair Decoration in the Southern Carpathian Basin. Temple Rings of the Ciumbrud and Donja Dolina Types
}

\author{
Marko Dizdar \\ Aleksandar Kapuran
}

\begin{abstract}
The Late Hallstatt period in the southern Carpathian Basin is marked by complex cultural relations for which the current knowledge is mostly based on the analyses of cemeteries. One of the most prolific forms of female jewellery in graves is bronze and silver temple rings that were used to decorate the head or hair. This is testified to by finds from the inhumation graves in Donja Dolina, where several pairs of temple rings, often of different forms, were located on both sides of the women's heads. Four basic types have been distinguished according to differences in the design of the terminals, and there are different variants according to the method of shaping the body. The oldest burial phases in Donja Dolina are characterized by smooth temple rings with a conical thickening at the terminals (Ciumbrud type), which have been found in the highest numbers in the inhumation graves in Transylvania. Temple rings of the Ciumbrud type from Donja Dolina should probably be seen as a reflection of established contacts and cultural transfer, but we should not completely rule out the possibility of the individual mobility of women. The reconstruction of how they were worn makes it possible to analyse the female bodily ornamentation, indicating that these are gender-specific items, which became an important part of the visual identity of women.
\end{abstract}

\section{Keywords}

Early Iron Age, southern Carpathian Basin, female head/hair jewellery, temple rings, female bodily ornamentation, cultural transfers.

Zusammenfassung - Späthallstattzeitlicher weiblicher Kopf-I Haarschmuck im südlichen Karpatenbecken. Schläfenringe der Typen Ciumbrud und Donja Dolina

Die Späthallstattzeit im südlichen Karpatenbecken ist durch komplexe kulturelle Zusammenhänge gekennzeichnet, deren Kenntnis derzeit vor allem auf der Analyse von Gräberfeldern beruht. Eine der verbreitetsten Formen des weiblichen Schmucks in den Gräbern sind bronzene und silberne Schläfenringe, die zum Schmuck des Kopfes oder der Haare verwendet wurden. Davon zeugen Funde aus den Körpergräbern in Donja Dolina, wo sich auf beiden Seiten des Kopfes der Frauen mehrere Paare von Schläfenringen, oft in verschiedenen Formen, befanden. Nach der unterschiedlichen Gestaltung der
Endstücke wurden vier Grundtypen unterschieden und nach der Art der Ausgestaltung des Körpers in verschiedene Varianten untergliedert. Die ältesten Bestattungsphasen in Donja Dolina zeichnen sich durch glatte Schläfenringe mit einer konischen Verdickung an den Enden aus (Ciumbrud-Typ), die besonders häufig in Körpergräbern in Siebenbürgen vorkommen. Schläfenringe des Ciumbrud-Typs aus Donja Dolina sind wohl als Zeichen von bestehenden Kontakten und Kulturtransfer zu werten, doch sollte auch die Möglichkeit der individuellen Mobilität der Frauen nicht völlig ausgeschlossen werden. Die Rekonstruktion der Trageweise der Schläfenringe ermöglicht eine Analyse des weiblichen Körperschmucks und deutet darauf hin, dass es sich um geschlechtsspezifische Objekte handelt, die zu einem wesentlichen Bestandteil der optischen Identität der Frauen wurden.

\section{Schlüsselbegriffe}

Hallstattzeit, südliches Karpatenbecken, weiblicher Kopf-/Haarschmuck, Schläfenringe, weiblicher Körperschmuck, Kulturtransfer.

\section{Time and Place}

The younger phase of the Early Iron Age (the Late Hallstatt phase) in the southern Carpathian Basin is represented by a highly heterogeneous material legacy. The southeast part of the Carpathian Basin is characterized by the development of the youngest phase of the Bosut group, singled out as the Syrmian group, which is known from research on settlements and chance finds that most often come from destroyed funerary complexes. ${ }^{1}$ Only a few cemeteries have been explored; they are small cemeteries with several inhumation burials. The only exception is the Szentlórinc

\footnotetext{
1 VASIĆ 1977. On the history of research and the relations between the Bosut, Dalj, and Syrmian groups, presenting the discussions so far: DizDAR 2019.
} 
cemetery in southeast Transdanubia, where a few cremation graves were also found. On the other hand, in the central part of the southern Carpathian Basin, the only systematically researched site is Donja Dolina located on the Sava River in northern Bosnia, which, after more than a hundred years, is still the largest excavated site in this region.

Since these cemeteries are dominated by inhumation burials, the graves of women stand out because of the richness and variety of the finds of costumes and jewellery, with inhumation burials allowing for the analysis of female bodily ornamentation, which has shown that these are gender- and often age-specific items. While there have been numerous analyses of female costume items, especially the characteristic open-work or astragal belts and diverse forms of fibulae, much less attention has been paid to jewellery. When it comes to jewellery items from the Late Hallstatt women's graves in the southern Carpathian Basin, the most common finds are necklaces composed of various glass beads, while there are fewer amber, coral, and gold and silver beads. There are a few examples of ring jewellery items, most notably different forms of bracelets; detailed analyses have been carried out for the lavishly decorated silver bracelets appearing at the end of the Early Iron Age. Ring jewellery can also include items that women used to decorate their head or hair. These are the finds of temple rings or hair ringlets, and very occasionally earrings. So far, little attention has been paid to this type of jewellery, especially the temple rings.

Given the scarcity of finds of jewellery items used by women to decorate their heads and hair in the southeast Carpathian Basin during the Late Hallstatt period, it will be of great importance for the analysis to include the cemetery in Donja Dolina, which has many well-dated funerary contexts, especially women's graves with various combinations of costume and jewellery items, particularly for the hair. Even today, more than a hundred years since the publication of the research, the settlement with the cemetery in Donja Dolina is still one of the most important sites of the Early Iron Age in the southern Carpathian Basin. Thanks to its location on important communication routes, it is rich with a heterogeneous material legacy in which local forms exist along with recognized influences from the southeastern Alpine region in the west, across the western and central Balkans in the south, to the Lower Danube in the east. For this reason, the items of female head/hair ring jewellery from Donja Dolina will be an excellent supplement to the ensemble of finds found at the sites in the southeastern Carpathian Basin, which are attributed to the Bosut or Syrmian group.
The biritual cemetery in Donja Dolina with more than 170 grave assemblages ${ }^{2}$ is still the starting point for studying the complex interactions that the Early Iron Age communities inhabiting the southern Carpathian Basin had with those from neighbouring areas. ${ }^{3}$ In the previous studies of the Donja Dolina cemetery, the emphasis was usually on the prestigious items of warrior defence equipment (helmets, shields) and bronze vessels. ${ }^{4}$ However, they also highlighted the noticeable appearance of certain items of female costume and jewellery that have their origin in those neighbouring areas. ${ }^{5}$ What distinguishes the cemetery in Donja Dolina from the other contemporary sites in the southern Carpathian Basin is the amalgamation of various items of female costume and jewellery into unique combinations not found anywhere else, even compared to some of the most significant sites in the neighbouring areas. In fact, in Donja Dolina, there is a noticeable emergence of hybrid combinations, often including objects with completely different origins within the same grave assemblage. Such combinations have always been, and still are, a mainstay in the chronological considerations of the Early Iron Age in the southern Carpathian Basin, with an importance far exceeding the chronological periodization of the site itself.

Some of the incentives for the implementation of the analysis were certainly the discussions about female hair jewellery from the area of the southern Carpathian Basin in the Late Bronze Age and the beginning of the Early Iron Age, which indicated the distribution of local forms, ${ }^{6}$ and it would be important to check this for the Late Hallstatt phase. On the other hand, analyses of female head/hair jewellery for a defined chronological framework, which were carried out for neighbouring areas - the northern Carpathian Basin ${ }^{7}$ and the Dolenjska region ${ }^{8}-$ also pointed to

2 Truhelka 1904. - Čović 1961. - Marić 1964, 19. - Teržan 1974, 43-45. - Čović 1987a, 232-233. - PARZinger 1992, 217-221 and Figs. 2, 6. - Gavranović 2011b, 126-127. - Gavranović 2016, 130. On the history of research of the Donja Dolina cemetery: Gavranović 2011b, 126-139.

3 TeržAn 1974, 43-45. - Čović 1987a, 281-284.

4 For instance: VAsIĆ 1975. - VAsić 1982. - TeRŽAn 1987a, 17. Teržan 1995. - Blečić Kavur, Pravidur 2012, 39-45. - Jašarević 2014. - Gavranović 2016, 127-129. - JašArević 2017.

5 Čović 1961. - Marić 1964. - TeržAn 1974, 43-45. - Čović 1987 a. - Teržan 1987a. - Gavranović 2011a.

6 VAsić 1988, 10-11 and Fig. 7. - VAsić 1996, 16-17 and Fig. 1. Metzner-Nebelsick 2002, 434. - Ložnjak Dizdar 2010, 162-163. - Pabst 2012, 413 and Map 44. - LožNJak Dizdar, Rajić Šikanjić 2016, 118-120 and Fig. 6. - Bojčić, LožNJAK DizDAR, HrŠAK 2018, 167-168.

7 Kemenczei 2002. - Ilon 2017. - Kozubová 2018. - Kozubová 2019.

8 Tecco Hvala 2007. - Tecco Hvala 2012, 327-333 and Fig. 122. Pavlovič 2018. 
decoration with local jewellery forms as an important part of highlighting various aspects of women's social identities.

However, before analysing the forms of female head/ hair jewellery from the sites located in the southern Carpathian Basin, it is necessary to look briefly at the chronological framework defined as the Late Hallstatt period, the latest analyses showing that this is an extremely complex issue.

The beginning of the Late Hallstatt phase in the southeastern Carpathian Basin is chronologically considered in different ways, both in terms of defining its inception, and in recognizing certain narrow timelines for a development of almost three hundred years. On the one hand, in the southeastern Carpathian Basin, where the development of the Early Iron Age is characterized by the material legacy of the Bosut group, the beginning of the younger phase can be defined as the end of the Basarabi phase around the middle of the $7^{\text {th }}$ century BC, which is the beginning of the 'fluted pottery phase'. This phase, defined primarily on the basis of the stratigraphy of the multilayer settlement of Gradina on the Bosut River, ${ }^{9}$ would last until the beginning of the Late Iron Age during the second half of the $4^{\text {th }}$ century BC. It is, therefore, an extremely long timeline. Interestingly, the contemporary finds of inhumation graves of the Bosut group, dating from the beginning of the $6^{\text {th }}$ century BC, have been attributed to the Syrmian group. However, the latest analyses considered these graves within the framework of the Bosut group, corresponding to the 'fluted pottery phase'. ${ }^{10}$ The western neighbours of the Bosut group are the sites located along the Danube, from the southeastern Transdanubia and Baranya to eastern Slavonia, southwestern Bačka and the westernmost parts of Syrmia, which are associated with the Dalj group, whose continuous development can be traced from the younger phase of the Urnfield Culture up to the end of the $7^{\text {th }}$ and the beginning of the $6^{\text {th }}$ century BC. This would mean that the beginning of the youngest phase of the Bosut group is contemporary with the last phase of the Dalj group, which is characterized by cremation burials. Of course, at the sites of both groups, especially in the settlements located in the contact zone (eastern Slavonia, western Syrmia), there was a permeation of ceramic forms and decorating techniques and motifs. On the other hand, in the central part of the southern Carpathian Basin, there are no sites that could be dated to the Late Hallstatt phase - except the settlement with the biritual cemetery in Donja Dolina, which is eponymous for the cultural group distributed

9 Medović, Medović 2011, 270-272 and Fig. 256.

10 On defining the beginning of the Late Hallstatt phase in the southeastern Carpathian Basin: Dizdar 2020. across the middle Sava valley and northern Bosnia. The oldest known closed funerary complexes from Donja Dolina date from the end of the first half of the $7^{\text {th }}$ century BC which is approximately the time when the Basarabi phase of the Bosut group ended - but the burials continued into the Late Iron Age. ${ }^{11}$

Thus, the Late Hallstatt phase in the southeastern Carpathian Basin is characterized by extremely complex cultural relations, which last until the beginning of the Late Iron Age, when there was a complete cultural reconfiguration of the observed area. It is a long period with shorter chronological determinants within it, which are based on the analysis of various forms of female costume items, primarily fibulae and belts. ${ }^{12}$ Late Hallstatt female costume items are not only a recognizable gender mark, but also a testimony to the networks and intensity of realized contacts with neighbouring or remote areas, and to different aspects of female social identity. Interestingly, jewellery items have not been the subject of special analyses so far, although they also stand out with their variety of forms. In fact, an important part of female body decoration, in addition to belts and fibulae, are those jewellery items that were worn on the head, most often as hair decorations - temple rings or ringlets -, whose analysis can also testify to established contacts and cultural transfers, perhaps even to the mobility of particular women. Also, the analysis of head/hair jewellery, as more than just a mark of the social status of the women who wore such jewellery, can perhaps point to the existence of an age concept, i.e. what was the most frequent age of the women who were buried with these forms of jewellery. Unfortunately, for the majority of graves we lack the results of anthropological analyses, so descriptions from the original publications are very important, as in the case of the Donja Dolina cemetery.

For the Late Hallstatt phase in the southeastern Carpathian Basin, there are several distinct forms of temple rings, which were made of bronze and only rarely of silver. Earrings occur very rarely, only as chance finds so far. ${ }^{13}$ The finds of temple rings are known from the graves at the cemetery in Donja Dolina, which stands out due to the number and variety of forms. However, the data from other sites are an important complement to the ensemble of finds included in the analysis.

As already stated, the custom of decorating the head and hair in the area of the southeastern Carpathian Basin has been

11 There have been finds of weapons and costumes, probably from destroyed graves, showing that the youngest graves were from the Middle La Tène: Dizdar 2018, 19-20.

12 Dizdar 2020.

13 VASIĆ 1991. 
documented in the cremation graves of women dated to the beginning of the younger phase of the Urnfield Culture, and continues until the beginning of the Early Iron Age, with the emergence of some new forms, particularly the double bronze wire temple rings with a pseudo-figure-of-eight terminal, singled out as the Velem-Szentvid type and the Vajuga-Pesak type. ${ }^{14}$ On the other hand, in the cemeteries of the Dalj group located along the Danube, there are numerous finds of simple and smooth bronze hair/temple rings with folded terminals of circular cross section and small diameter. Since these are cremation graves, where hair/temple rings sometimes show traces of burning, it is not quite clear how they were worn on the head. ${ }^{15}$ However, the discovery of inhumation grave 91 at the Dalj group cemetery in Sotin, where the buried adult woman had four bronze hair/temple rings on her head (probably on a textile band or headcovering) of the kind appearing in those cremation graves, indicates the likely way in which they were worn. ${ }^{16}$ The finds from the aforementioned grave, as well as numerous others from the cemeteries of the Dalj group, show that adult women decorated their hair with a few simple and smooth bronze hair/temple rings. Likewise, these finds show that there was a long tradition of decorating hair with various forms of temple rings in the southeastern Carpathian Basin, which would continue - in other forms - until the end of the Early Iron Age and even into the beginning of the Late Iron Age. With the end of the Basarabi phase of the Bosut group, and with the end of the Dalj group a little later, all the previously known forms of bronze temple rings disappeared, and new forms appeared which had been unknown until then. This change probably took place at the end of the first half of the $7^{\text {th }}$ century BC, which corresponds to the identified changes in female costume items and other ring jewellery - in other words, there was a noticeable cultural reconfiguration, which is clearly seen in the female bodily decoration. The time of the change and the potential sources of the new forms can probably (or at least partially) be explained by

14 Vasić 1988, 10-11 and Fig. 7. - Popović, Vukmanović 1998, 30-31 and Fig. 27. - MetZner-Nebelsick 2002, 434. - LožNJaK Dizdar 2010, 162-163. - РABST 2012, 413 and Map 44. - On the example of inhumation grave 202 from the Vukovar-Lijeva bara cemetery, with bronze hair/temple rings worn on a hairband, and some other finds of spiral hair/temple rings from cremation graves: VINSKI, VINSKi-GASPARINI 1962, 271 and Pls. III/48-53; IV/56, 59.-On Batina-type temple rings in the cemeteries of the Dalj group as an eastern influence at the beginning of the Early Iron Age: MetZner-NebeLSICK 2002, 433-439 and Pl. 8/26-27. - KemenCZei 2002, 63-64. - Kemenczei 2009, 80. - Kozubová 2018, 31-32. - Kozubová 2019, 109. 15 Bojčić, LožnJak Dizdar, HRŠaK 2018, 167-168.

16 Ložnjak Dizdar, Hutinec 2013, 9 and Fig. 3. - Ložnjak DizDAR, RAJIĆ ŠIKANJIĆ 2016, 118. the analysis of female hair jewellery, particularly from the inhumation graves in Donja Dolina, where the position of the temple rings is exactly known: always on both sides of the head of the deceased women over the several centuries of their wearing. The cemetery in Donja Dolina also stands out for its large number of different forms of temple rings, which are usually made of bronze; silver temple rings are exceptions which appear only in the final burial phases. The finds from Donja Dolina have an important complement in the finds from other sites in the southeastern Carpathian Basin attributed to the Syrmian group, which will also be included in the future analysis. It must be pointed out that the analyses of female head and hair jewellery have been carried out for the neighbouring areas where the appearance of local forms is noticeable. Can the local nature of female head/ hair jewellery during the Late Hallstatt be recognized in the southern Carpathian Basin too? Are there certain rules in the composition of head decoration jewellery with regard to the age of the deceased and/or her social status on the basis of the ensemble of other finds? Aside from the local jewellery, can we recognize forms that originated in other areas? If so, is there a difference in the way they were worn between the different areas in which they appear? Can we identify the mechanisms of their spreading, and whether they were based on cultural transfers or the mobility of specific women who wore certain forms of temple rings? Are there chronologically recognizable forms? These questions, as well as those that will emerge during the analysis, may be answered by an examination of the entire ensemble of the known finds dated to the Late Hallstatt phase from the sites located in the southern Carpathian Basin.

\section{Typological Classification of the Late Hallstatt Temple Rings in the Southern Carpathian Basin}

As we already pointed out, the most numerous forms of female head decoration jewellery are different forms of temple rings that were probably used to decorate the hair. When attempting to define and reconstruct the way in which they were worn, it is very important to consider the finds from the inhumation graves which show that those items were probably worn in the hair. Temple rings are usually made of bronze, but silver ones have been recorded, which follow the bronze specimens in form and decoration.

The known finds of temple rings from the sites in the southern Carpathian Basin, with the cemetery in Donja Dolina as the main source of data, ${ }^{17}$ can be classified into several

17 We owe special thanks to Andrijana Pravidur, Ph.D., from the National Museum in Sarajevo, who let us examine the finds of the temple rings from the Donja Dolina cemetery. 


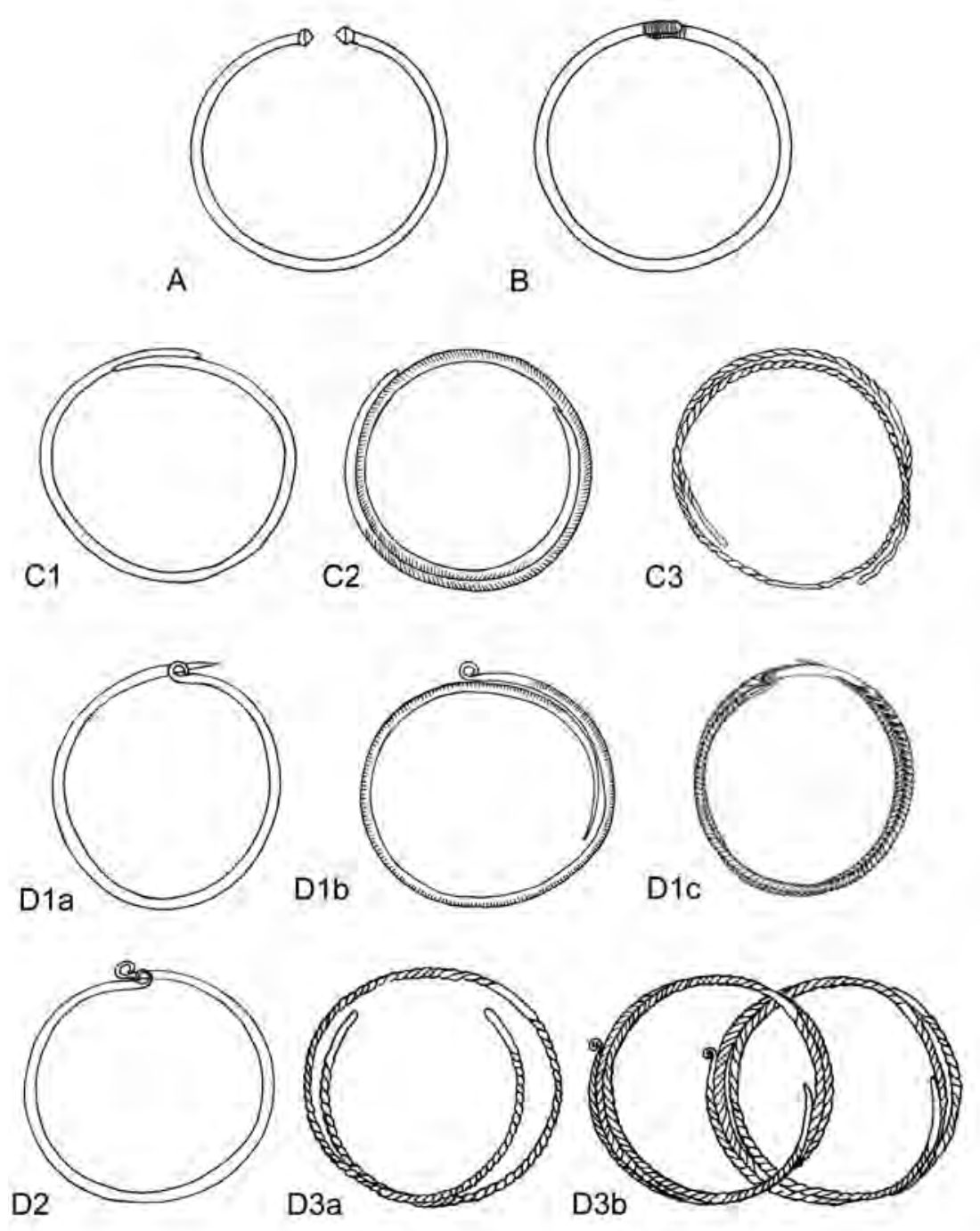

Fig. 1. Types of temple rings in the Donja Dolina cemetery (drawing: A. Kapuran).

basic types, primarily according to the design of their terminals (Fig. 1). Specific types can then be further classified into variants according to the body-shaping method, which can be: 1) smooth; 2) ribbed on the outside; 3 ) twisted. Also, the analysis focuses on the dimensions of the temple rings. ${ }^{18}$ Regarding the proposed classification of temple rings, it is important to point out that the earlier insights from the previous analyses of the finds from Donja Dolina were taken

18 In the cemetery in Donja Dolina, along with basic types with variants, there are some finds with an unclear function. The shape and production method indicate that these may be objects used as head/ hair decorations, although other purposes are not excluded. This group may also include the few items originating from neighbouring areas and being characteristic of other cultural groups, notably the Dolenjska and Vekerzug groups. into account; ${ }^{19}$ the most detailed one is the classification proposed by Mario Gavranović, who recognizes three basic types - with a conical thickening at the terminals; with one folded terminal; with tapering terminals - which correspond to the basic forms distinguished here. ${ }^{20}$

We distinguish four basic types of temple rings with variants (Fig. 1), with special importance given to the funerary assemblages containing different basic types and variants:

- type A: smooth temple rings with a conical thickening at the terminals - Ciumbrud type - that are

19 Marić 1964, 32-44. - Čović 1987a, 241-258. - Marić and Čović recognize the temple rings that correspond roughly to the singled-out types.

20 Gavranović 2011a, 216-217 and Fig. 217. 
further classified according to the material from which they were made: variant A1: bronze temple rings; variant $\mathrm{A} 2$ : temple rings made in precious metals (gold, silver);

- type B: temple rings with slightly widened and straight or conical terminals, which are decorated with transverse grooves - Donja Dolina type;

- type C: temple rings with tapering terminals that are further classified according to the body-shaping method: variant $\mathrm{C} 1$ : smooth temple rings; variant C2: temple rings that are ribbed on the outside; variant C3: temple rings with a twisted body;

- type D: temple rings where at least one terminal is hammered and folded; type D is further classified into variants according to the body-shaping method. Type D1 includes temple rings with one terminal which is hammered and folded: variant D1a: smooth temple rings; variant D1b: temple rings that are ribbed on the outside; variant D1c: temple rings with a twisted body. Type D2 includes temple rings where both terminals are hammered and folded. Type D3 includes silver temple rings that can be classified into two basic variants: variant D3a: temple rings with a twisted body and slightly widened terminals; variant D3b: temple rings with a twisted body and one terminal that is hammered and folded. ${ }^{21}$

It should certainly be pointed out that some temple rings, particularly those from the cemetery in Donja Dolina, have not been preserved completely, and it is sometimes difficult to determine the decorating method on the body of a temple ring. Therefore, it is not always possible to unambiguously determine the type and the variant. Also, the first publication of the finds from Donja Dolina sometimes has discrepancies between the descriptions and the drawings of temple rings, both in the form and manner of decoration and in the dimensions. However, the classification of the temple rings from Donja Dolina allows the identification of the proposed basic typological features, with an important complement in the form of the other finds from the southern Carpathian Basin and the identified analogies. In the chronological identification of recognized variants, particular importance is given to the contexts, which are primarily graves, where the temple rings appear with costume items

21 Considering the complexity and the considerable scope of the topic, as well as the long period of time in which the temple rings were worn, here we will analyse only the A and B types, which are often found together in the same grave assemblages, while other forms will be presented in future analyses. (e.g. belts, fibulae) and other items of ring jewellery (e.g. torcs, bracelets, pendants, various forms of beads).

\subsection{Type A: Temple Rings with a Conical Thickening at the Terminals - the Ciumbrud Type}

The first recognized form of temple rings is represented by type A (the Ciumbrud type), which is characterized by a smooth body of round cross section, ending with a conical thickening at both terminals.22 The terminals can be slightly spaced, touching, or slightly overlapping. Interestingly, different terminals sometimes appear together in the same grave in Donja Dolina. The terminals overlap to a greater extent in rare cases, such as a temple ring from grave M. Petrović Jr. 3 (Fig. 3/1), but that temple ring has a smaller diameter, so it was probably folded to a greater extent later. ${ }^{23}$ Based on the material from which they were made, two variants can be distinguished - variant A1 is represented by bronze temple rings, while variant $\mathrm{A} 2$ includes temple rings made in precious metals (gold, silver), which is unknown in Donja Dolina, but has been recorded in Transylvania and northeast Hungary.

In the southern Carpathian Basin, bronze temple rings of variant A1 have been found only in the Donja Dolina cemetery, where they have been identified almost exclusively in the inhumation graves of women - a total of 13; the only cremation grave is grave M. Petrović 2 (Fig. 5). Temple rings have been found on both sides of the head of the deceased, at the height of the temples - usually several pairs, indicating the manner in which they were worn. Importantly, when Ćiro Truhelka described inhumation grave N. Čegrlja 3 (Fig. 2) and grave 1 in the garden of N. Šokić, he concluded that temple rings (which he called temple hoops) hung on a cap or head scarf worn by the deceased. ${ }^{24}$ Different forms have also been found in the settlement at Gradina in Donja Dolina, including one temple ring of variant A1 dated after Zdravko Marić to phases IIa, IIb and even into phase IIc. ${ }^{25}$

22 Truhelka 1904, 85-135, 145-146 describes the thickenings as pear-like.

23 For the temple rings of types A and B found in the Donja Dolina cemetery, the data on other items of costume and jewellery in the funerary complexes are provided in Tabs. 1 and 3.

24 Truhelka 1904, 84-85 and Fig. 65, Pl. XL. - Čović 1987a, 241. - Gavranović 2011a, 216. - A similar opinion is provided by PARZINGER 1992, 217, who concludes that the temple rings were worn on both sides of the head as ear or hair decorations: PARzinger 1992, 217, 220 and Fig. 2.

25 Marić 1964, 33, 36, 40 and Pls. VI/15; XVI/17. - Marić dates the appearance of temple rings to phase Ic and looks for their source in forms from the end of the Urnfield Culture, such as the example from cremation grave M. Petrović Jr. 46, which he later dated to phase IIa: MARIĆ 1964, 31-32. - The same opinion is given by Čović 1987a, 240 and Pl. XXIV/14-15. However, these are probably the remains of the bracelet in the mentioned grave. 


\begin{tabular}{|c|c|c|c|c|c|c|c|c|c|c|c|c|c|}
\hline 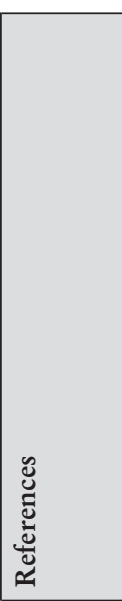 & 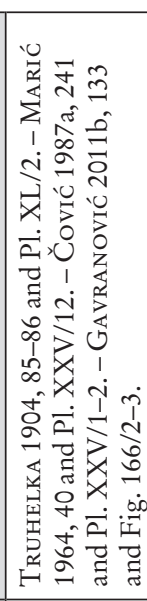 & 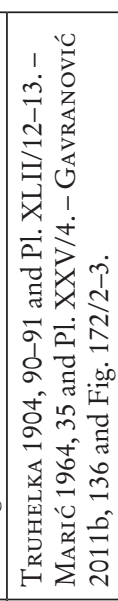 & 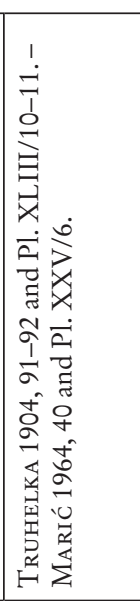 & 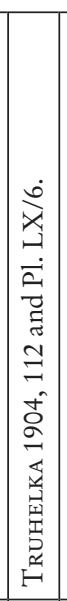 & 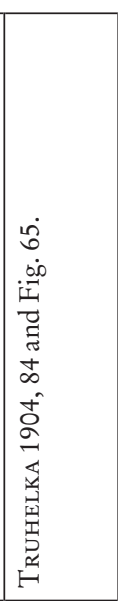 & 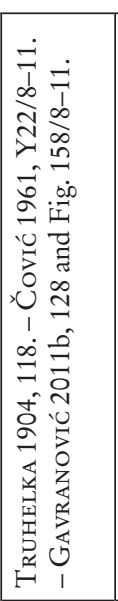 & 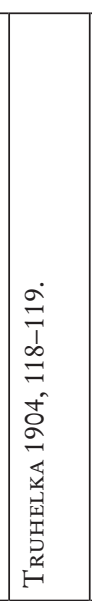 & 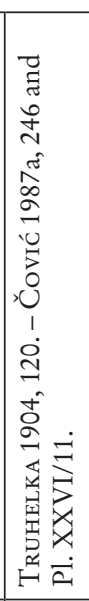 & 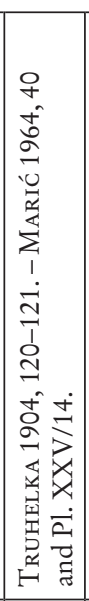 & 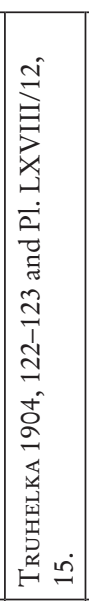 & 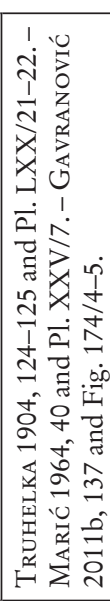 & 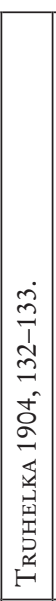 & 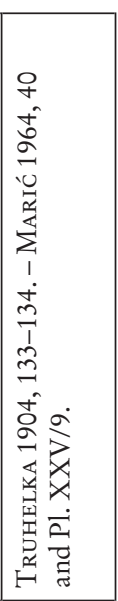 \\
\hline 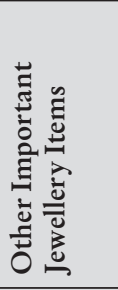 & 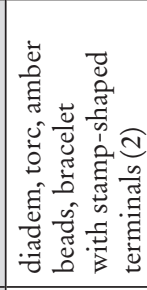 & 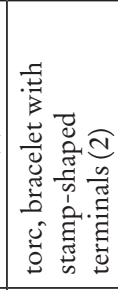 & 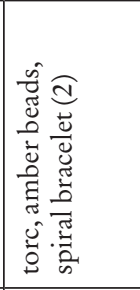 & & 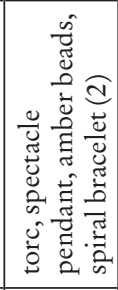 & $\begin{array}{l}\widehat{1} \\
0 \\
0 \\
0\end{array}$ & 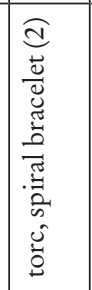 & & 亗 & 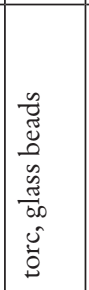 & 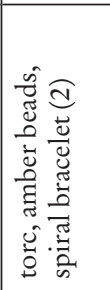 & & 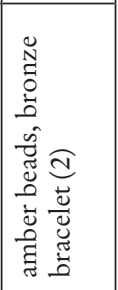 \\
\hline 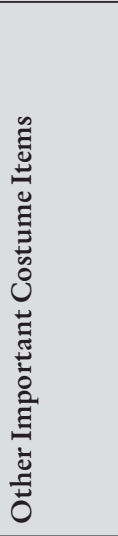 & 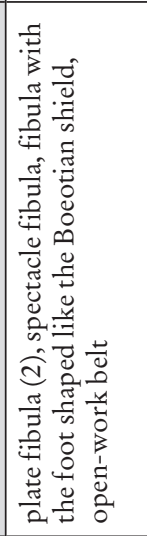 & 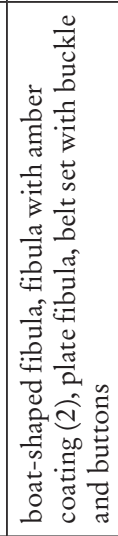 & 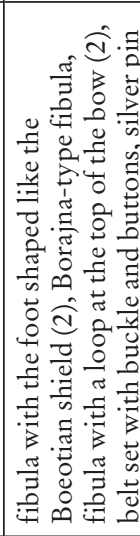 & & 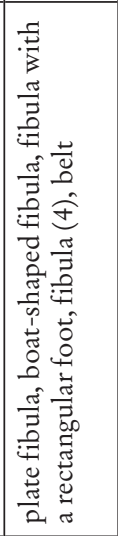 & 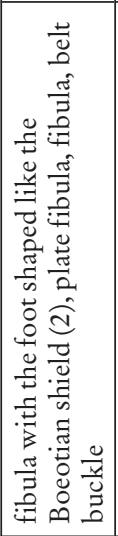 & 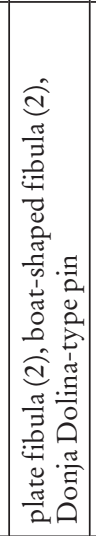 & & 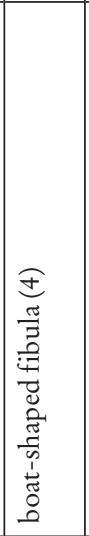 & 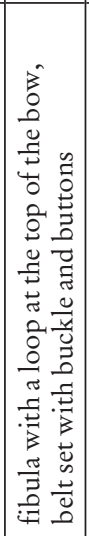 & 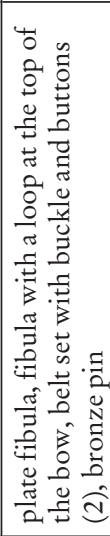 & & 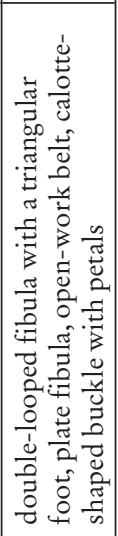 \\
\hline 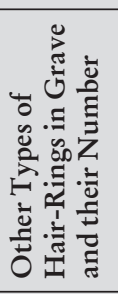 & 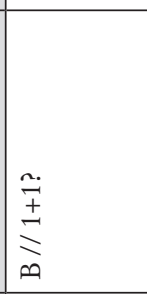 & 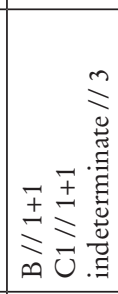 & 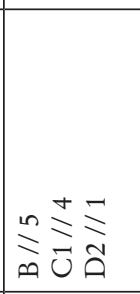 & & & $\frac{\Xi}{\bar{U}}$ & $\stackrel{x}{\stackrel{x}{u}}$ & $\underset{⿱}{ \pm}$ & $\frac{m}{m}$ & 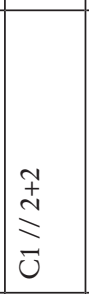 & & $\begin{array}{l}\Xi \\
\Xi \\
\vec{u}\end{array}$ & 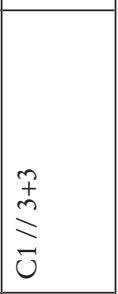 \\
\hline 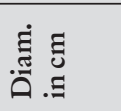 & $\begin{array}{l}0 \\
\infty\end{array}$ & $\underset{b}{\stackrel{f}{f}}$ & $\stackrel{\sim}{\sim} \stackrel{\infty}{\infty}$ & $\hat{n}$ & \begin{tabular}{|l}
$n$ \\
$\infty$ \\
$\infty$
\end{tabular} & 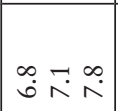 & 웃 & $\begin{array}{l}\text { Or } \\
\text { in }\end{array}$ & $\stackrel{n}{n}$ & $\begin{array}{l}\overrightarrow{0} \\
\vdots \\
\sim \\
i n \\
i n\end{array}$ & $\begin{array}{l}a \\
\hat{i} \\
\vdots \\
0 \\
\dot{0}\end{array}$ & & o \\
\hline$\dot{z}$ & $\stackrel{m}{m}$ & $\stackrel{9}{ \pm}$ & $\exists$ & - & 孚 & $\stackrel{\Xi}{\sim}$ & $x$ & $\nexists$ & $\exists$ & $\stackrel{\text { I }}{\sim}$ & 乐 & $\exists$ & \pm \\
\hline$\stackrel{8}{4}$ & 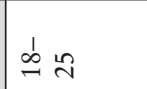 & $\frac{\vec{z}}{\underset{\sigma}{\sigma}}$ & $\frac{\vec{z}}{\vec{J}}$ & & $\stackrel{1}{I} n$ & $\frac{\vec{t}}{\vec{z}}$ & 莺 & 莺 & 矛 & $\frac{\vec{Z}}{\vec{\Xi}}$ & 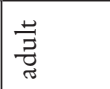 & \begin{tabular}{|l|} 
\\
\multirow{\sigma}{*}{} \\
\end{tabular} & $\frac{\vec{J}}{\vec{z}}$ \\
\hline 丞 & 㐏 & 壳 & 㐏 & $\overrightarrow{\mathrm{E}}$ & 㐏 & 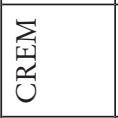 & 壳 & 旁 & 穵 & 壳 & 壳 & 壳 & 空 \\
\hline$\vec{\sigma}$ & 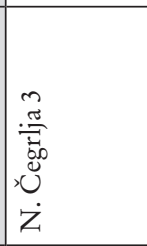 & 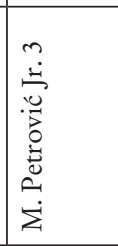 & 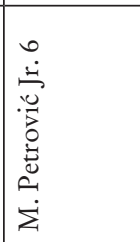 & 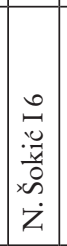 & 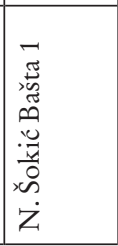 & 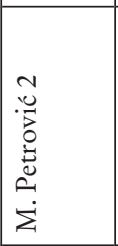 & 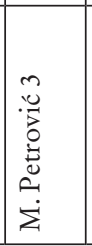 & 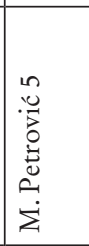 & 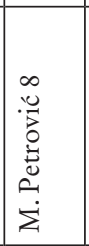 & 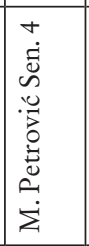 & 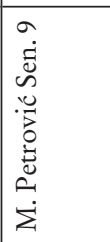 & 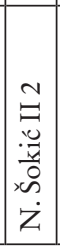 & 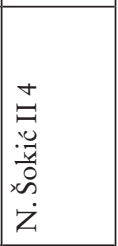 \\
\hline
\end{tabular}



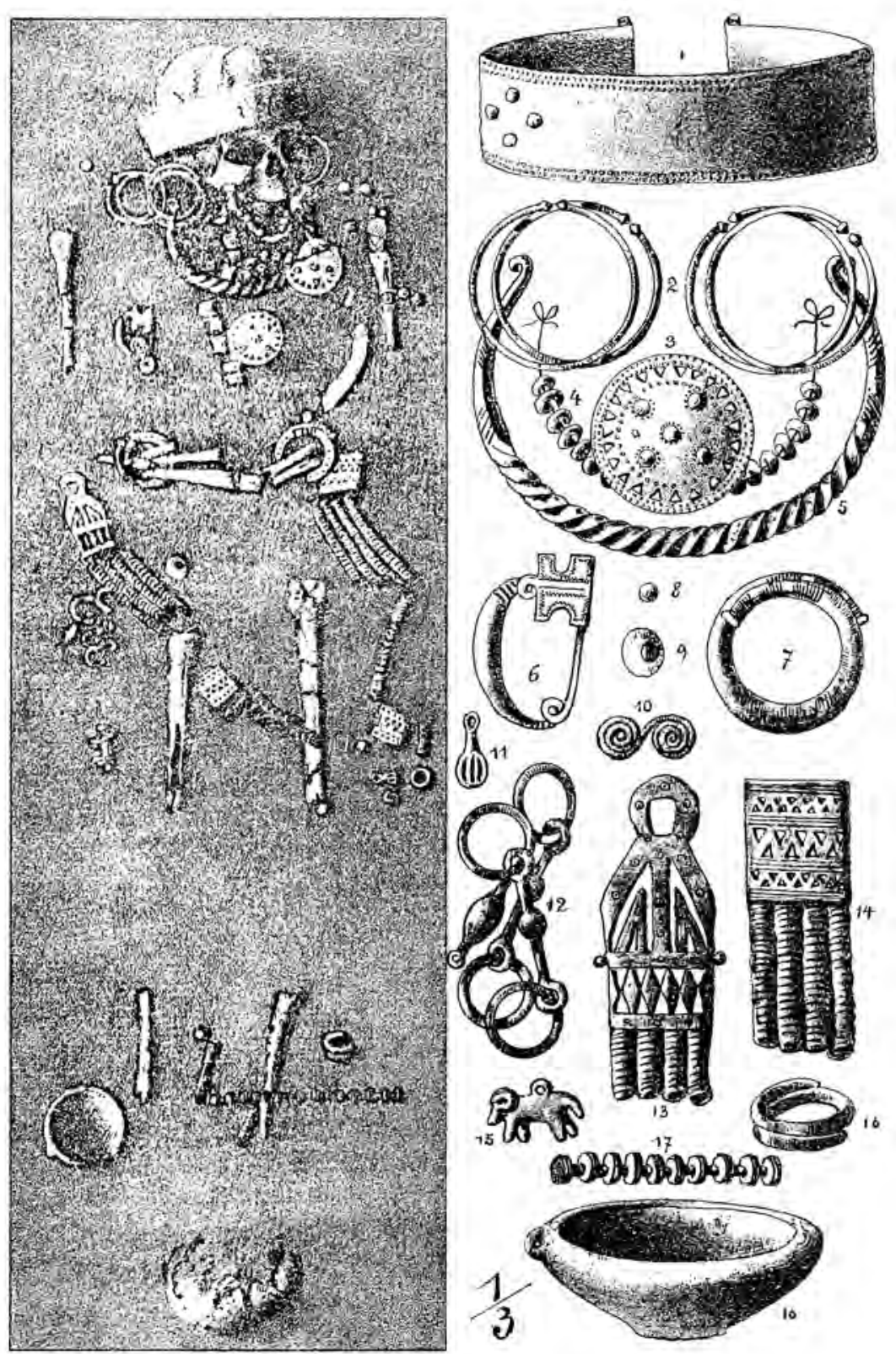

Fig. 2. Grave N. Čegrlja 3 (after Truhelka 1904).

Variant A1 temple rings in the Donja Dolina cemetery rarely appear in graves as the only form; ${ }^{26}$ as a rule, they are accompanied by type $\mathrm{B}$ and much more frequently by type $\mathrm{C} 1$ (Tab. 1). Also, the graves sometimes contain a combination of three different basic forms of temple rings (types A1, B, C1); furthermore, in grave M. Petrović Jr. 6 (Fig. 4/5), they seem to be joined by a type D2, where both

26 As the only shape in grave M. Petrović Sen. 9. Also, the grave N. Šokić I 6 actually represents a pit in which a single variant A1 temple ring was found, probably originating from a destroyed grave. terminals are hammered and folded (Tab. 1). Another interesting feature is the manner of wearing type A temple rings. When they are the only form in the grave, there are three or four type A temple rings on each side of the head (Tab. 1); when there is a single type A temple ring on each side of the head, it is usually combined with type B and/ or $\mathrm{C} 1$; when there are two on each side, they are combined with type C1 (Fig. 6/1-2), and when there are three, it seems that they were combined with a single type B on each side, as in grave N. Čegrlja 3 (Fig. 2/2). As shown by all these examples, there is almost always the same number on each 


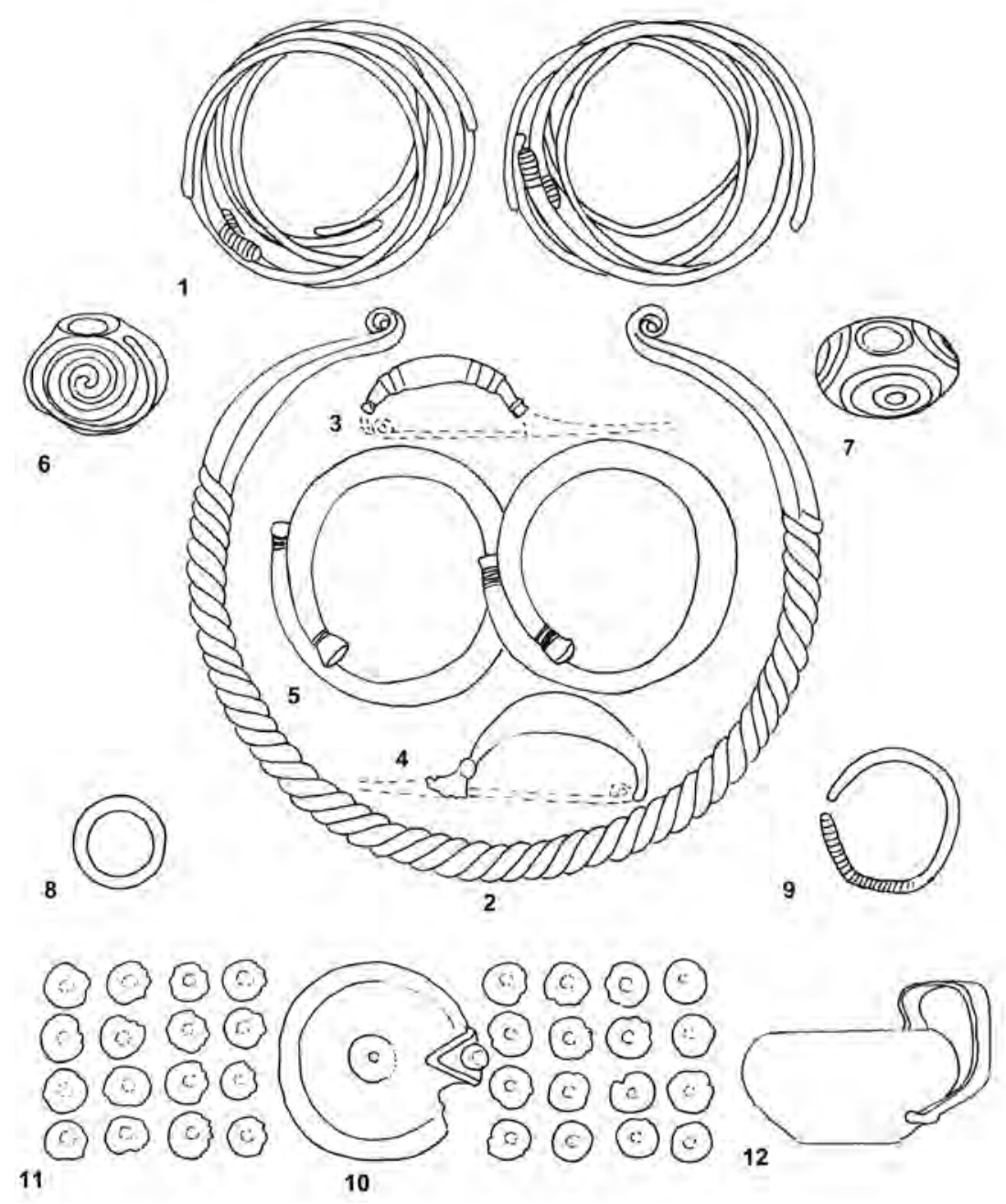

Fig. 3. Grave M. Petrović Jr. 3 (after Truhelka 1904).

side of the head. The radius of temple rings ranges between 4.7 and $10.5 \mathrm{~cm},{ }^{27}$ but most frequently falls between 7.0 and $8.5 \mathrm{~cm}$ (Tab. 1).

In the cemetery of Donja Dolina variant A1 temple rings were found in the graves of women, often richly furnished with items of costume and ring jewellery (Tab. 1). The highest number of these graves belongs to phase $2 \mathrm{a}$ after the periodization of Borivoj Čović, or phases 1 and 2 according to Biba Teržan (Figs. 2-3, 5, 9). The younger group of graves with variant $\mathrm{A} 1$ temple rings were dated to phase $2 \mathrm{~b}$ after

27 The variant A1 temple ring with the diameter of $10.5 \mathrm{~cm}$ from grave N. Šokić 2 which was dated to phase IIc: MarIć 1964, 40 and Pl. XIV/17.
Čović cr $^{8}$ or phase 3 after Teržan ${ }^{29}$ (Figs. 4, 6-7). The finds of temple rings from the graves in Donja Dolina were classified in detail by Gavranović. He dated the ones with thickening conical terminals to the horizon of double-loop fibulae with the foot in the shape of a Boeotian shield, i.e. to the second half of the $7^{\text {th }}$ and the beginning of the $6^{\text {th }}$ century BC. ${ }^{30}$ The

28 Čović 1987a, 238-251 and Pls. XXV/1-2; XXVI/11. - According to Čović, phase 2a corresponds to subphase Stična 1 in Dolenjska or the Glasinac phase IVc-1: Čović 1987a, 242-244. Phase 2b corresponds to subphase Stična 2 or the Glasinac IVc-2 phase: Čović 1987a, 249-251.

29 TeržAn 1974, 43-45 and Fig. 7.

30 Gavranović 2011a, 216-217, 268 and Fig. 217/1. - Gavranović 2016, 132 and Fig. 4. - Gavranović dates the oldest graves in Donja Dolina to the late $8^{\text {th }}$ and the early $7^{\text {th }}$ century BC: Gavranović 2011a, 263-265. - GAVRANOvić 2016, 130-132. 


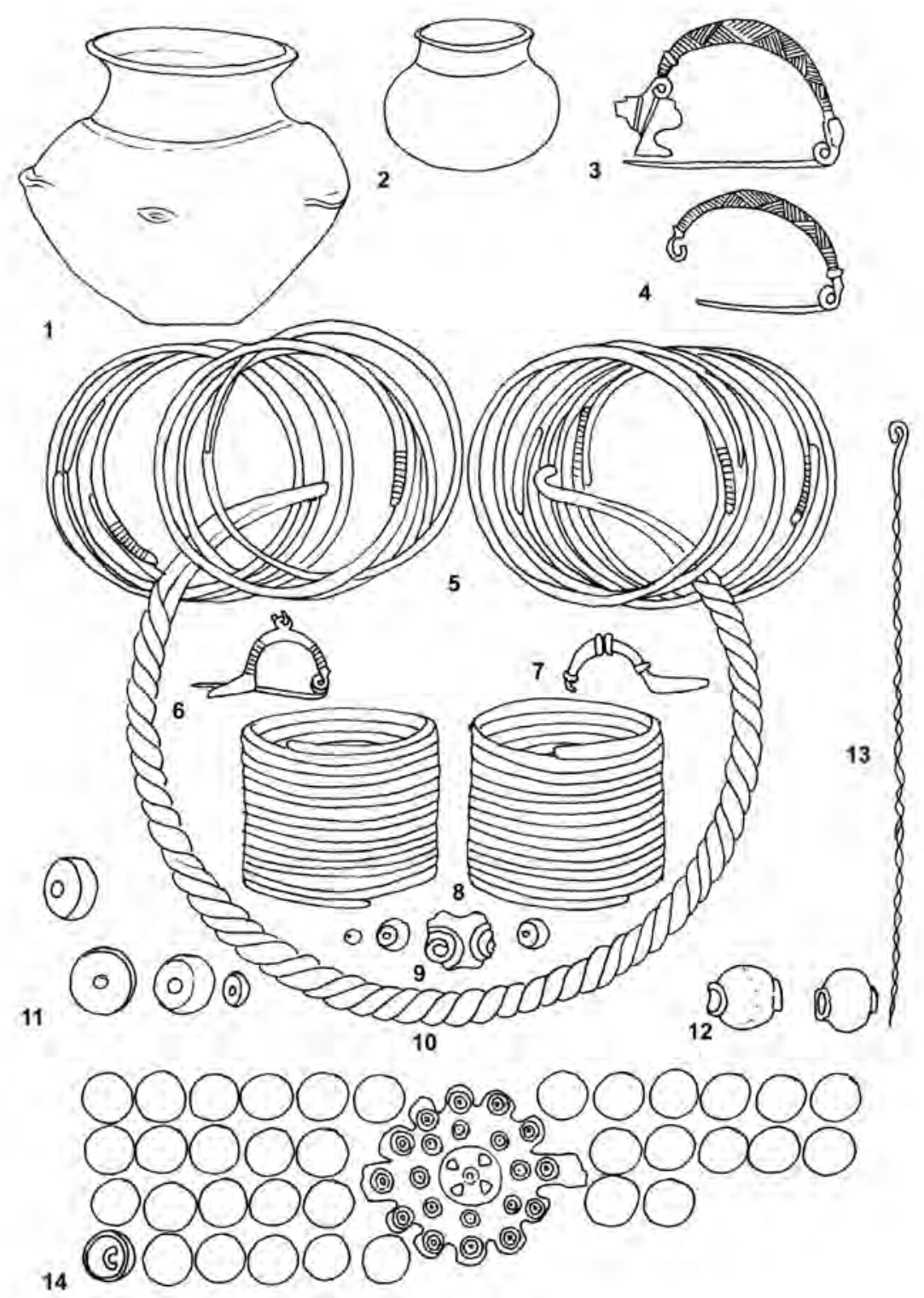

Fig. 4. Grave M. Petrović Jr. 6 (after Truhelka 1904).

dating of the variant A1 temple rings will enable detailed typo-chronological analyses of other items of costume and ring jewellery from these graves, which will also include the graves with type $\mathrm{B}$ temple rings (Tabs. 1,3).

The nearest find of variant A1 temple rings from the Donja Dolina cemetery probably comes from a grave in Dobra (Kožica I) at Đerdap/Iron Gate (Fig. 10). The group of finds were initially defined as a small hoard dated to $\mathrm{Ha} C$; aside from two temple rings (then defined as bracelets), it contained a bracelet decorated with transverse grooves at the terminals, two bronze fragments of rounded cross section, and fragments of two double-loop fibulae. The temple rings are rounded in cross section and have spaced terminals that end with small conical thickenings. ${ }^{31}$ Later, Rastko Vasić assumed that these were probably found in an inhumation grave. One fibula has been associated with double-loop fibulae with a ribbed bow and probably an hourglass-shaped foot - this form would be dated to the beginning of the Early Iron Age. The other fragment, hexagonal in cross section with decorated ends, is associated with double-looped fibulae with the foot in the shape of a Boeotian shield, which seems to date the first fibula to a later period. Vasić also points out that the rings with conical thickening terminals are not bracelets but probably hair rings or temple rings. ${ }^{32}$

31 TAsić 1969. - Popović 1984, 135 and Fig. 1.

32 VAsić 1999, 58, 66 and Pls. 30/360; 34/446. 


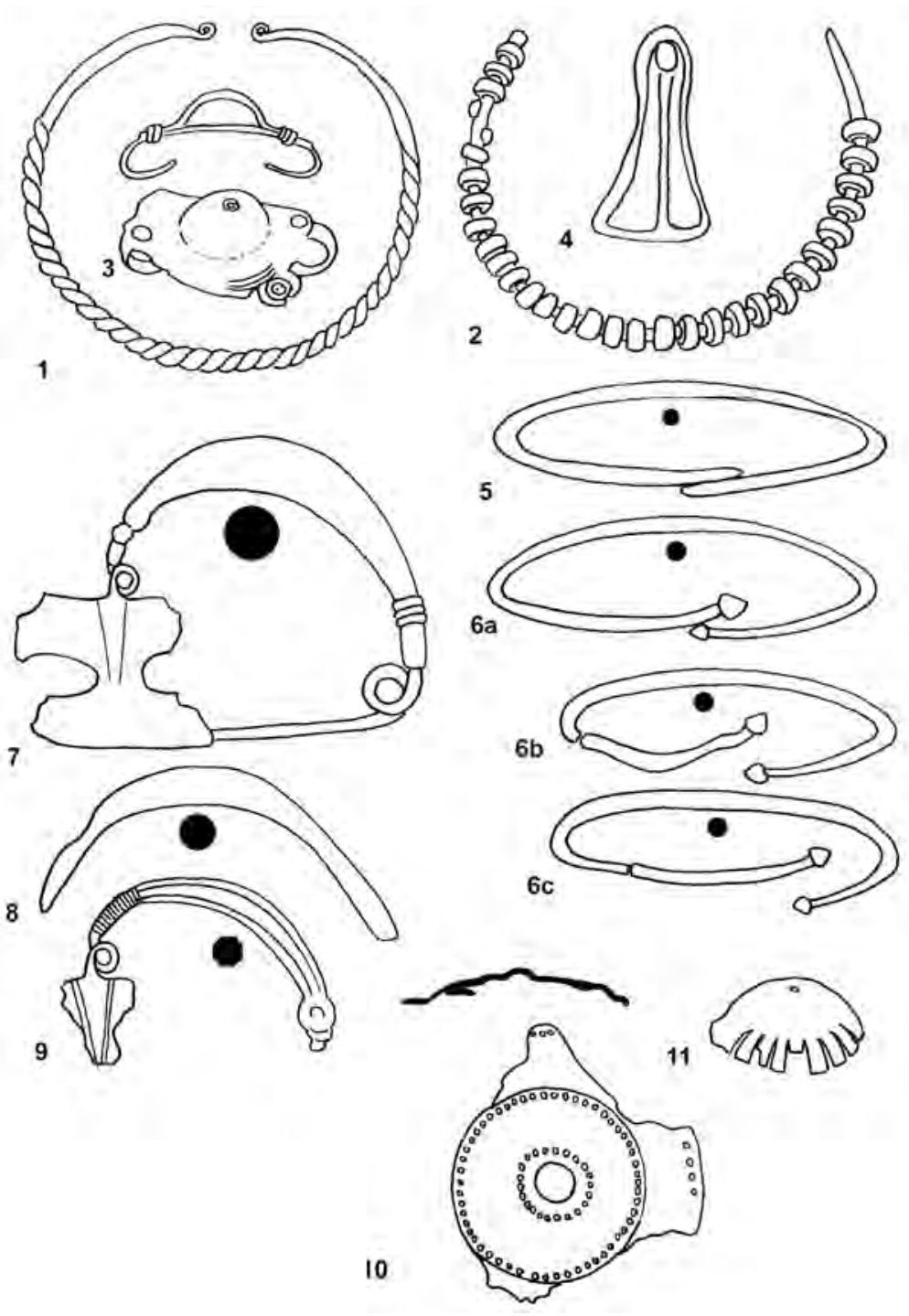

Fig. 5. Grave M. Petrović 2 (after Gavranović 2011b).

\subsubsection{Temple Rings of Type A - the Ciumbrud Type - in Tran- sylvania and Northeast Hungary}

After the analysis of the Donja Dolina cemetery with the finds of variant A1 temple rings, we come to some interesting insights about other sites with temple rings of this type. First of all, these sites are quite far from Donja Dolina. In fact, finds from Transylvania and northeast Hungary are direct parallels of the finds from Donja Dolina, raising the question of their relationship, just as there are interesting insights about the different manners in which they were worn. ${ }^{33}$

33 The connection of the finds of A1 temple rings from Donja Dolina with the finds from eastern Hungary, that is, with the Szenetes-Vekerzug cemetery, was already mentioned by Marić $1964,40,42$.
The greatest number of rounded cross-section bronze temple rings with thickening conical terminals has been found in the Ciumbrud group inhumation graves that were distributed across Transylvania (Fig. 16) during the Early Iron Age (Tab. 2), ${ }^{34}$ which is why they have been defined as Ciumbrud-type temple rings. ${ }^{35}$ Unlike the female graves in Donja Dolina, which often contain several variant A1 temple rings on each side of the head or combined with other types, the Ciumbrud group inhumation graves always have

34 For the finds of type A temple rings from the Ciumbrud group cemeteries, Tab. 2 provides the information on their diameter, the material from which they were made, and other finds of costume and jewellery items in the grave assemblages.

35 Kozubová 2018, 31-32. - Kozubová 2019, 107 and Fig. 31. 

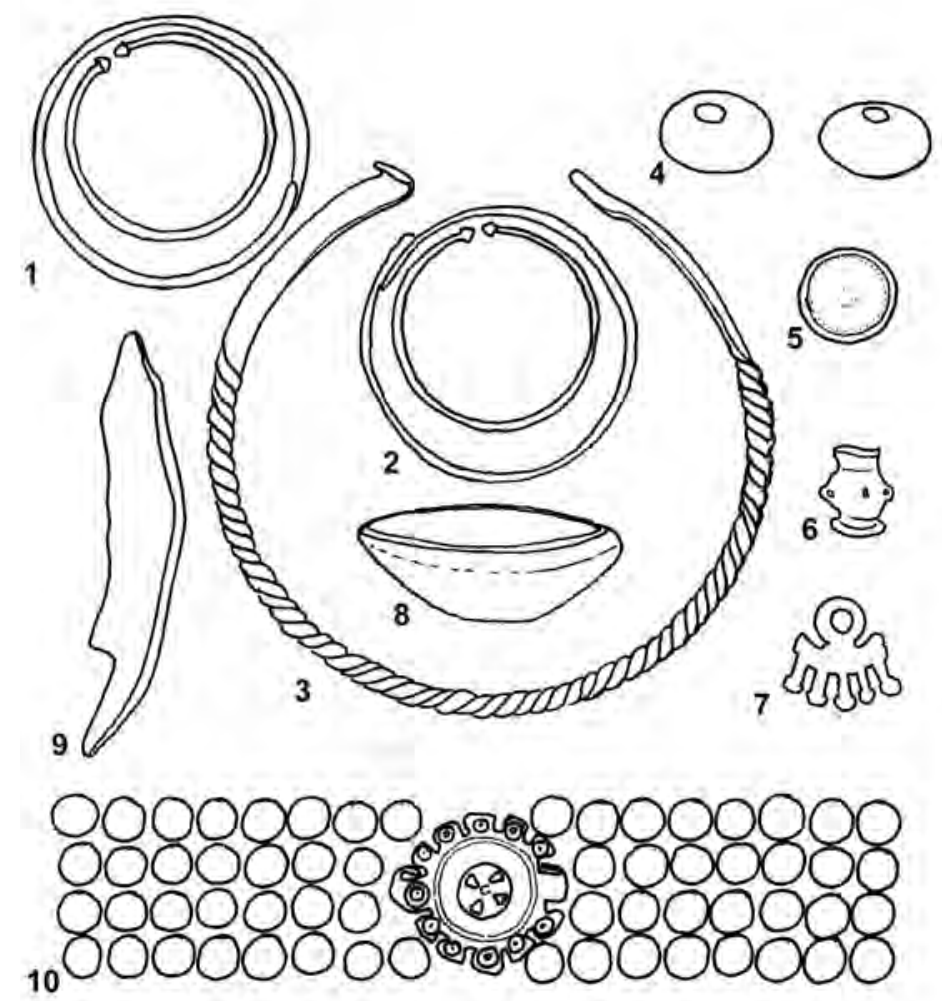

Fig. 6. Grave M. Petrović Sen. 4 (after Truhelka 1904).
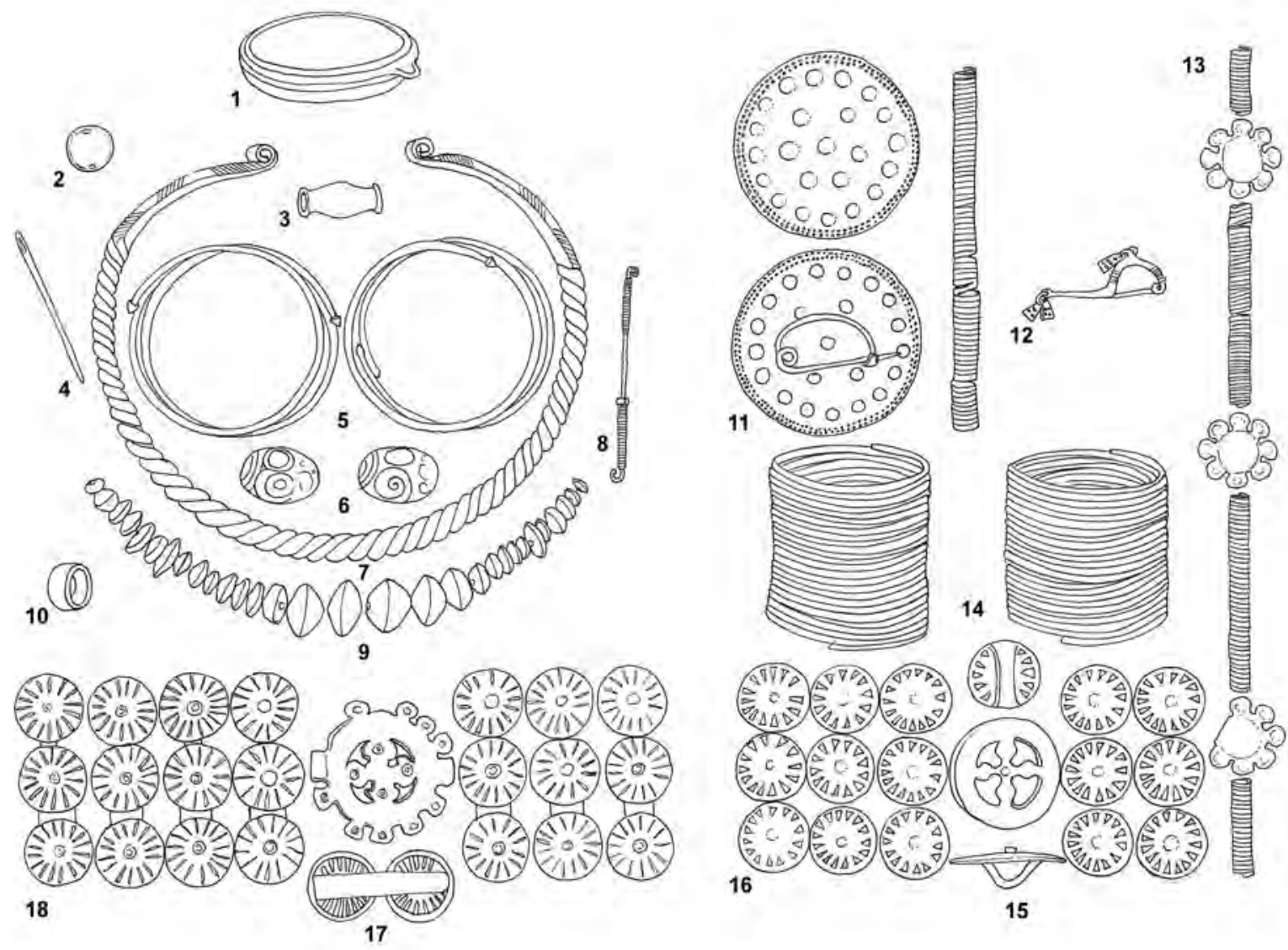

Fig. 7. Grave M. Petrović Sen. 9 (after Truhelka 1904). 


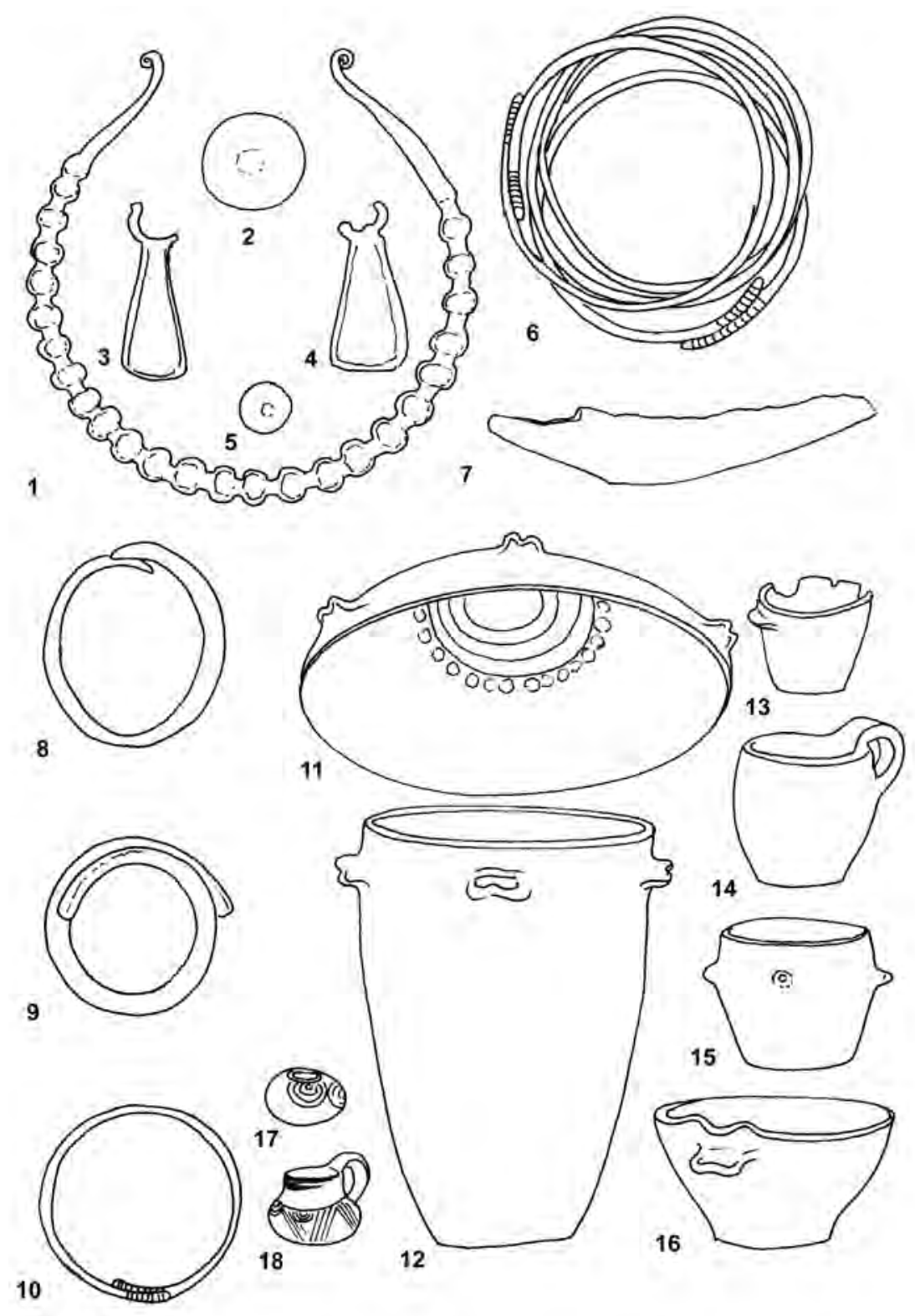

Fig. 8. Grave S. Jakarić 14 (after TRUHELKa 1904).

a single type A temple ring on each side of the head, almost always as the only type. Sometimes the grave contains just one temple ring of this kind. As a rule, they are made in bronze - variant A1, and only rarely in precious metals (gold, silver) - variant A2.

The eponymous cemetery of Ciumbrud-Podireu includes inhumation grave XIV with a buried adult woman; on each side of her head there was a single bronze temple ring of the variant A1. ${ }^{36}$ Again without a context, probably

36 Ferenczi 1966, 54 and Fig. 18/2. - Ferenczi 1969, 50 and Fig. 18/2. from destroyed graves, there are finds of variant A1 temple rings. ${ }^{37}$ Inhumation grave IV at the Cipău-Gară cemetery contained a bronze temple ring with one preserved conical thickening (Fig. 12). ${ }^{38}$ Grave 7 at the Cristești-Szörtsey cemetery also contained a bronze temple ring. ${ }^{39}$ In inhumation

37 Ferenczi 1965, 87 and Fig. 8/2, 5, 12-14.

38 Vlassa 1961, 28 and Fig. 6/3. - Kemenczei 2004, 79, 81 and Fig. 2/7.

39 Crişan 1965a, 59 and Fig. 12/7. - Kemenczei 2004, 83 and Fig. 3/11. - The grave also contained two spectacle fibulae without a central figure-of-eight loop: CRIŞAN 1974, 107 and Fig. 6/2. - KEMENCZEI 2004, 83 and Fig. 3/10. 

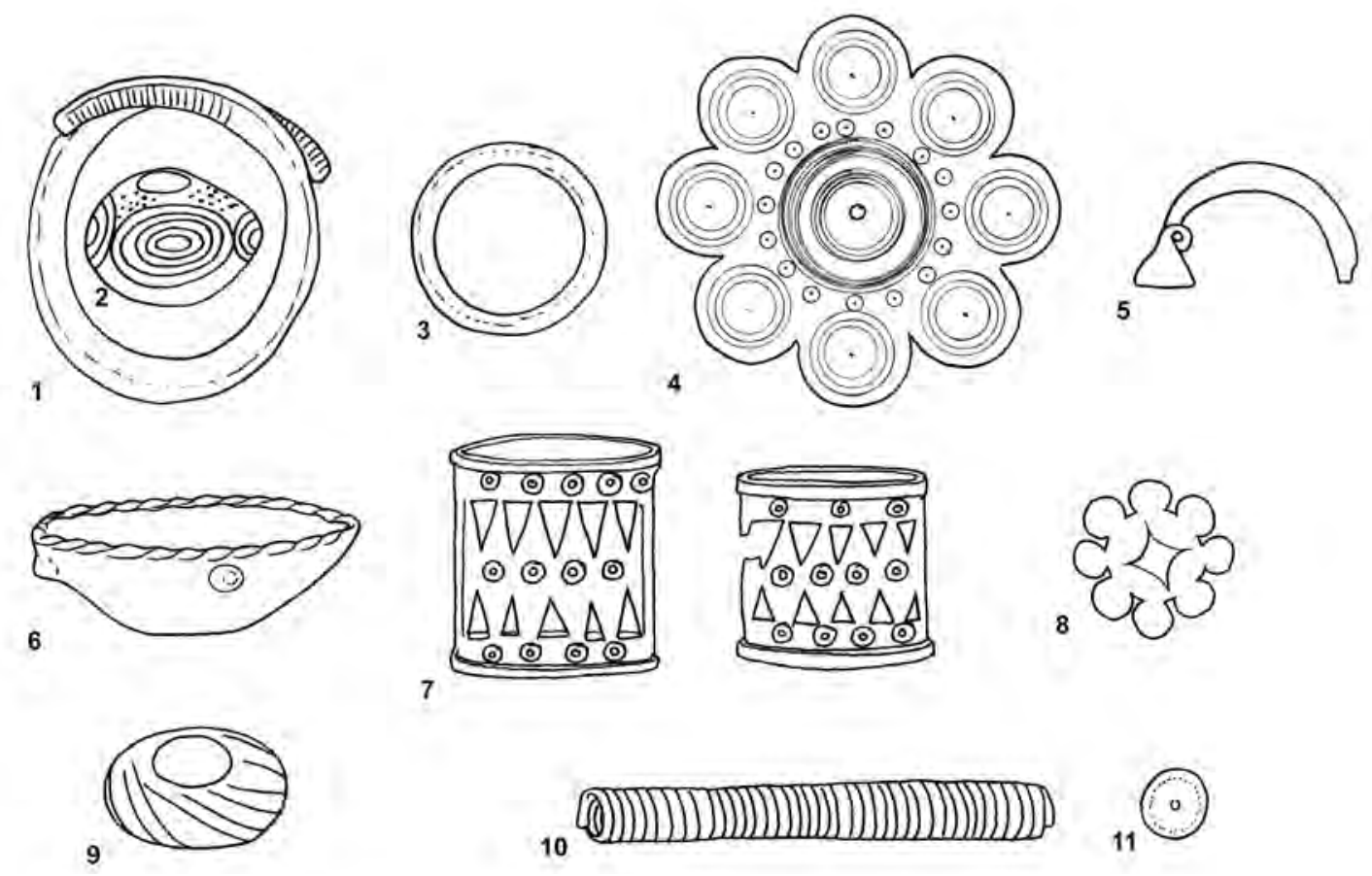

Fig. 9. Grave N. Šokić II 4 (after TruhelKa 1904).
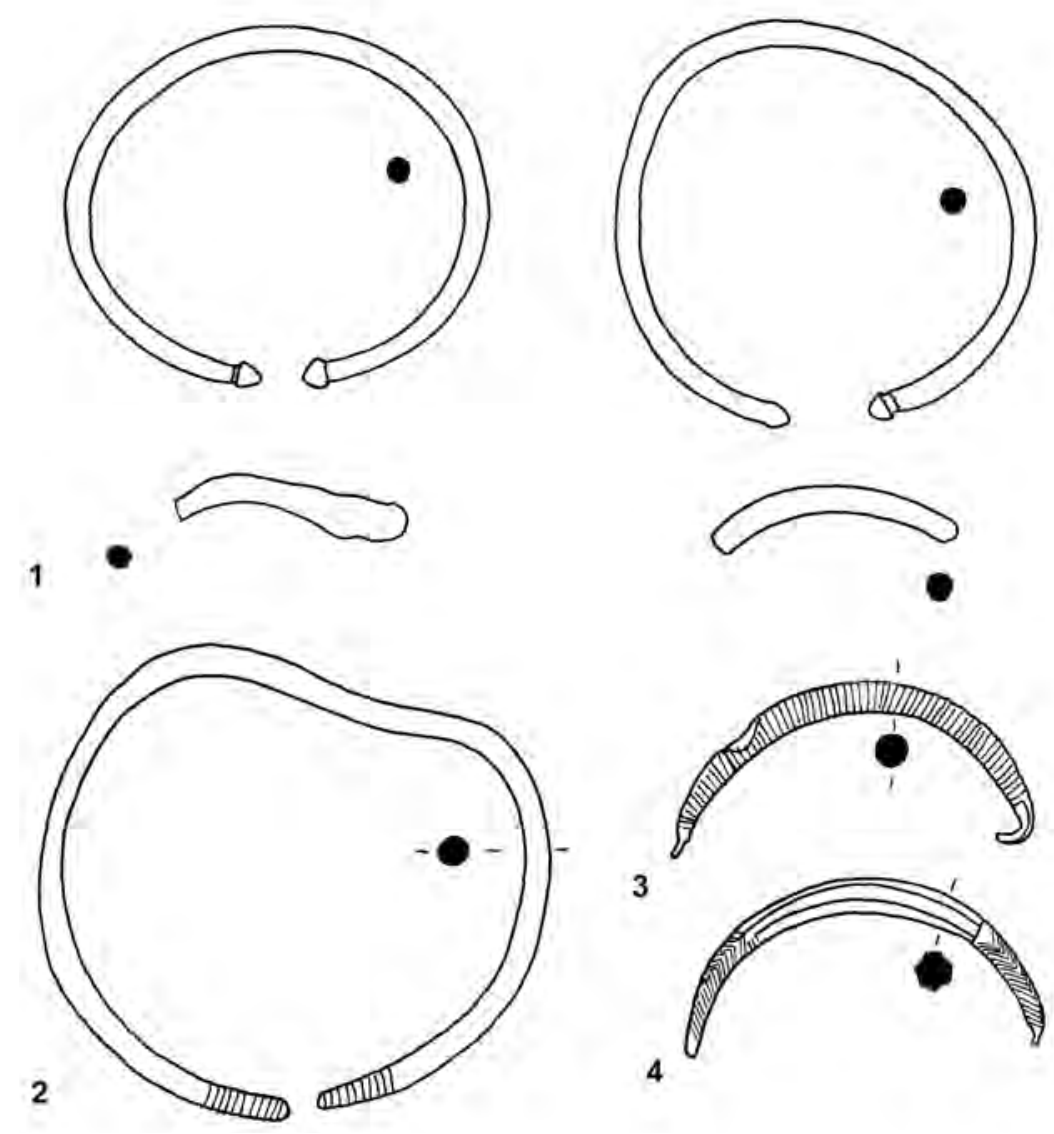

Fig. 10. Finds from Dobra-Kožica I (after Popović 1984). 
grave 10, where an adult woman was buried, there was a single temple ring on each side of the head..$^{40}$ In the Blaj cemetery, in inhumation grave 1 , probably containing a girl aged 8 to 10 , a fragmented bronze temple ring was found, ${ }^{41}$ while inhumation grave 2 , where an adult woman was buried, contained two temple rings. ${ }^{42}$ Grave 3 is interesting; it is assumed that a man was buried there. On the left side of the head there was a temple ring. ${ }^{43}$ In inhumation grave 7 , containing an adult woman, a single temple ring was found on each side of the head..$^{44}$ For the research of type A temple rings, particular significance is given to the Ozd-Piscul Deagului cemetery where 16 inhumation graves have been found. Graves 1-2 contained a bent temple ring with thickening terminals. ${ }^{45}$ Grave 8 is particularly important; it contained an adult woman with two temple rings (Fig. 13) which were found next to the head of the deceased. ${ }^{46} \mathrm{We}$ should also mention the damaged grave 16, where a woman was buried too, and which contained a fragment of a temple ring. ${ }^{47}$

Aside from the finds in grave assemblages, there are also a large number of finds of bronze temple rings with conical thickenings for which the context of the find is unknown, even though most of them probably originated from destroyed graves ${ }^{48}-$ Dezmir $^{49}$ and the Oradea-Salca cemetery. ${ }^{50}$ At several locations in the Aiud there were inhumation graves, some of which also contained type A temple rings; not far from that is the eponymous cemetery of $\mathrm{Ci}^{-}$ umbrud-Podireu, which gave the name to the group that is thought to have formed in the middle of the $7^{\text {th }}$ century BC. The site of Aiud-Parc included a bronze temple ring, but also three fragments of a gold temple ring (variant A2) with a conical thickening terminal. ${ }^{51}$ Also, what was probably a

40 ZRÍNYi 1965, 37 and Pls. V/3; XIII/37; XV/38.

41 Vasiliev 1972, 22 and Pl. V/1.

42 Vasiliev 1972, 26 and Pl. VI/1-2. - Vasiliev 1980, 105 and Pl. $18 / 14$.

43 VAsiliev 1972, 26 and Pl. VII/6.

44 Vasiliev 1972, 27 and Pl. IX/1-2. - On the golden objects from this grave: VAsiliev 1970, 53-55, Figs. 3/1-6; 5/1-2. Including the parallels in the Szentes-Vekerzug cemetery and in the area north of the Black Sea.

45 Vasiliev, ZRínYi 1974, 90 and Pl. X/1.

46 VAsiliev, ZRínyi 1974, 93 and Pls. Vb; IXa; XII/6-7.

47 Vasiliev, ZRínyi 1974, 96 and Pl. XVI/16.

48 E.g. Transylvania: VAsiliev 1980, 105 and Pl. 19/5.

49 Crişan 1964, 92 and Fig. 3/2, Pl. II/1.

50 Németi 1982, 130 and Fig. 20/4. - According to the data in: CRIŞAN 1965b, 136.

51 CrişAn 1974, 107 and Fig. 5/7. - VAsiliev 1980, 105 and Pl. 19/1.Vulpe 1984, 36, 38 and Figs. 2/3; 7/9. - These are probably the finds from several destroyed graves. double grave contained temple rings. ${ }^{52}$ At the Aiud-Gerepen site there was also a temple ring. ${ }^{53}$ Grave 20 in the nearby Gîmbaș-Măgauricea cemetery contained four temple rings and a Poiana-type fibula. ${ }^{54}$ Grave 14 included two temple rings. ${ }^{55}$ Inhumation grave 7 in the Teiuş-Cetăţuia cemetery, in which an adult woman was buried, contained two temple rings next to the head. The first temple ring has one terminal with a conical thickening and one in the form of a hemisphere. Both thickenings of the other temple ring are hemispherical. ${ }^{56}$ Not far from the mentioned grave, another two inhumation graves were found. Grave 1 contained a fragment of a temple ring next to the head of an adult woman. ${ }^{57}$ Grave 2 - another burial of a woman - contained a silver temple ring (variant A2) with expanding and overlapping conical terminals. ${ }^{58}$

Very important insights into the manner of wearing temple rings have been gained in the exploration of the Budești-Fînațe cemetery dated from the middle to the end of the $7^{\text {th }}$ century BC. Grave 1 contained an adult woman who had a single temple ring on each side of her head (Fig. 11).$^{59}$ In the richly furnished grave 3, a girl aged 9 to 10 was buried with a temple ring next to the left ear. Alexandru Vulpe dates this grave to before $600 \mathrm{BC} .{ }^{60}$ Also, grave 5 , in which an adult woman was buried, contained a single temple ring on each side of the head. ${ }^{61}$ Mărișelu is another important cemetery which has been dated from the end of the $7^{\text {th }}$ to the middle of the $6^{\text {th }}$ century BC. Grave 2, probably the burial of an adult woman, included a single temple ring on each side of the head. ${ }^{62}$ Grave 3, another burial of an adult woman, included

52 Vulpe 1984, 40 and Fig. 6/15-19.

53 Vulpe 1984, 43-44.

54 Vulpe 1984, 47 and Fig. 8/4-6, 8. - Kemenczei 2004, 90 and Fig. 4/17.

55 Kemenczei 2004, 89 and Fig. 4/20, 22

56 HoREDT 1953, 802 and Fig. 11/1-2.

57 Vasiliev, Badea, Man 1973, 28 and Fig. 3/1.

58 Vasiliev 1970, 41-42 and Fig. 1/3. - Vasiliev, Badea, Man 1973, 28, 31 and Fig. 4/3. - CrişAn 1974, 107 and Fig. 5/9. - VAsiliev 1980, 105 and Pl. 19/2. - Vulpe 1990, Pl. 46C. - Chochorowski 1998, 480 and Fig. 3/52. - Chochorowski 2014, 21, 23 and Fig. 11/52. A similar spherical pendant was found in grave M. Petrović Sen. 10: Truhelka 1904, 125 and Pl. LXXII/20. As one of the oldest graves of the Ciumbrud group: CHOchorowski 2014, 23.

59 Marinescu 1984, 48 and Fig. 2A/1a-b.

60 Marinescu 1984, 48 and Fig. 3/8. - Vulpe 1990, 98, 128 and Pl. 42B. - There are three open-work pendants, with the parallel in grave M. Petrović Sen. 10: Truhelka 1904, 125 and Pl. LXXII/21. MARINESCU 1984, 79-80: it also mentions a parallel with a find from tumulus III of the Cepari-Topliţa site and the Kisravazd hoard in western Hungary, dated to the turn of the $7^{\text {th }}$ and $6^{\text {th }}$ centuries BC: FEKETE 1973, 341 and Fig. 3/30.

61 Marinescu 1984, 48 and Fig. 4/4a-b.

62 Marinescu 1984, 49 and Fig. 8A/2a-b. 

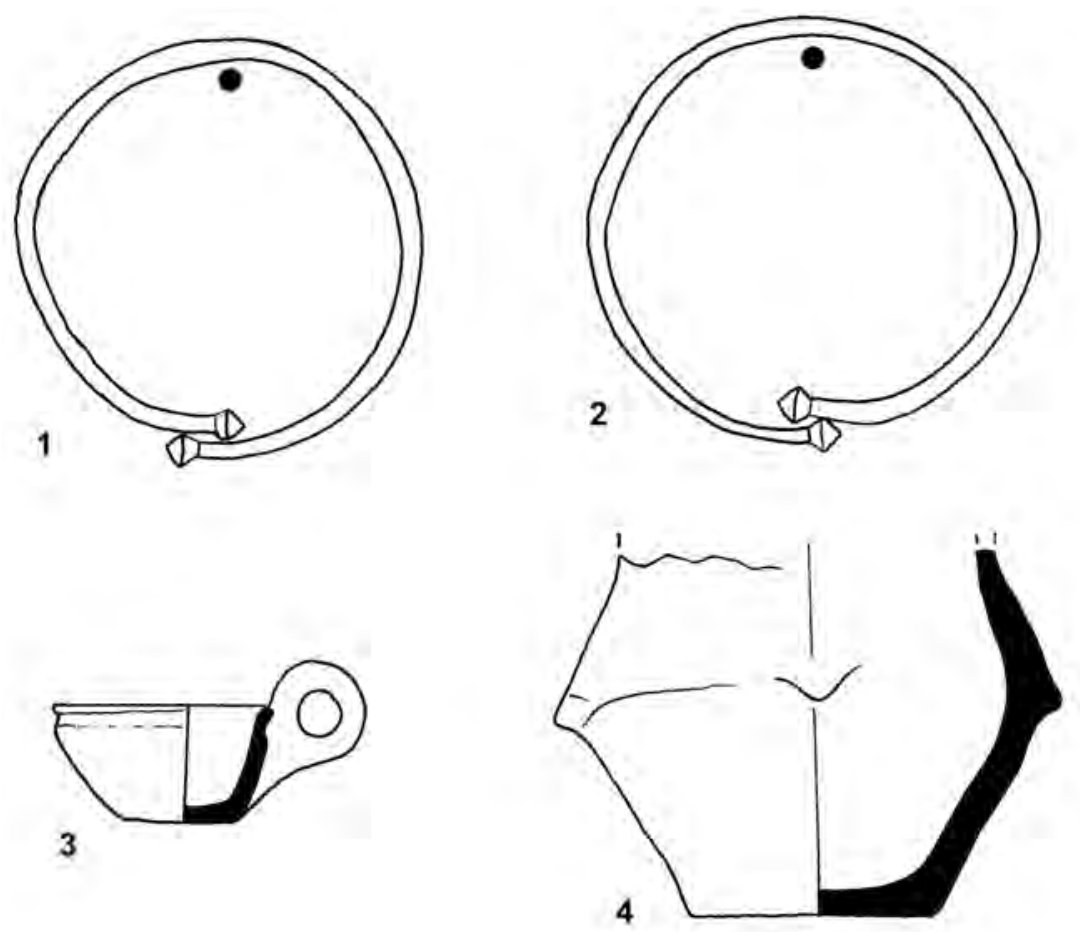

Fig. 11. Grave 1 from the Budești-Fînațe cemetery (after MARINEsCu 1984).

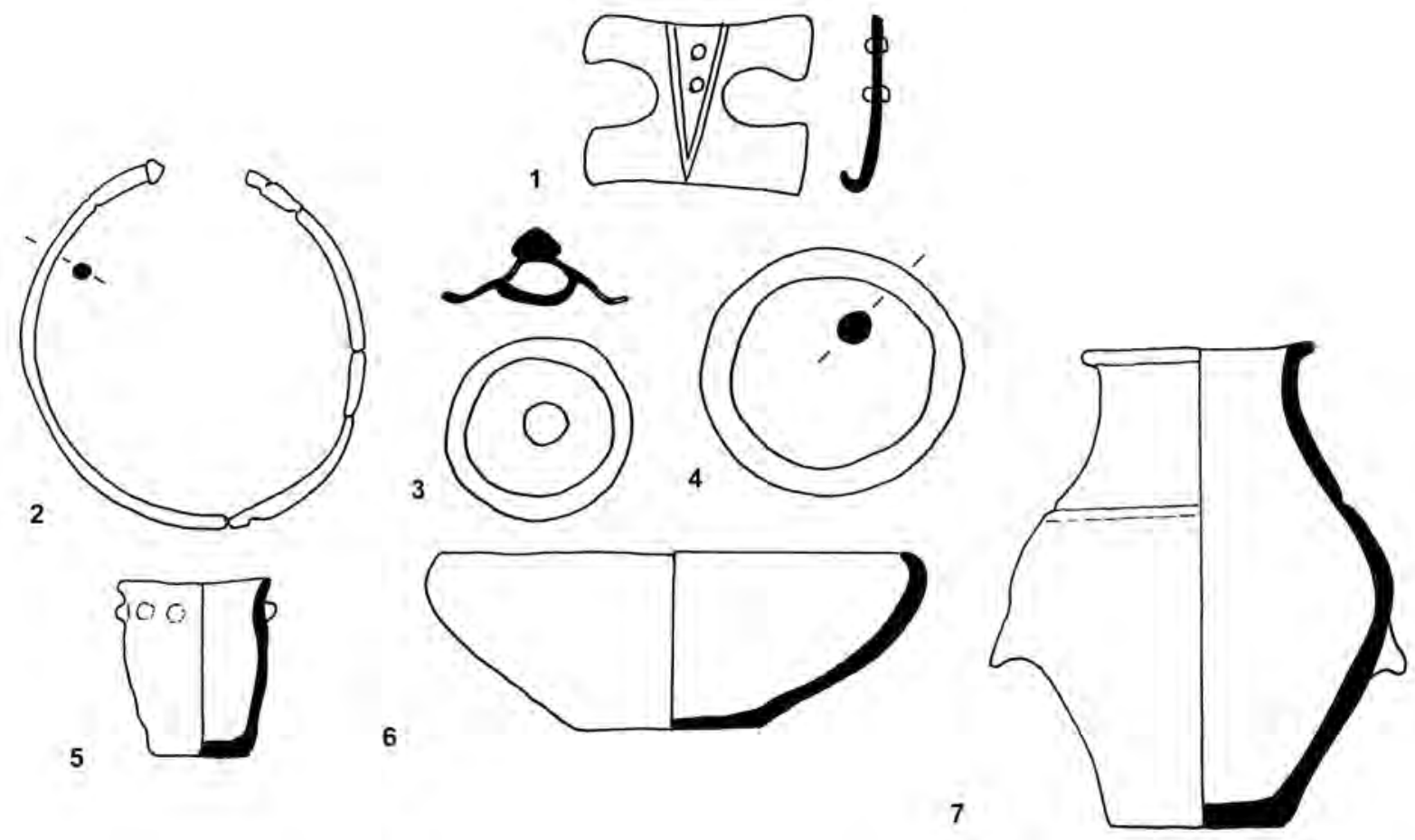

Fig. 12. Grave 4 from the Cipău-Gară cemetery (after KemenCZei 2004). 

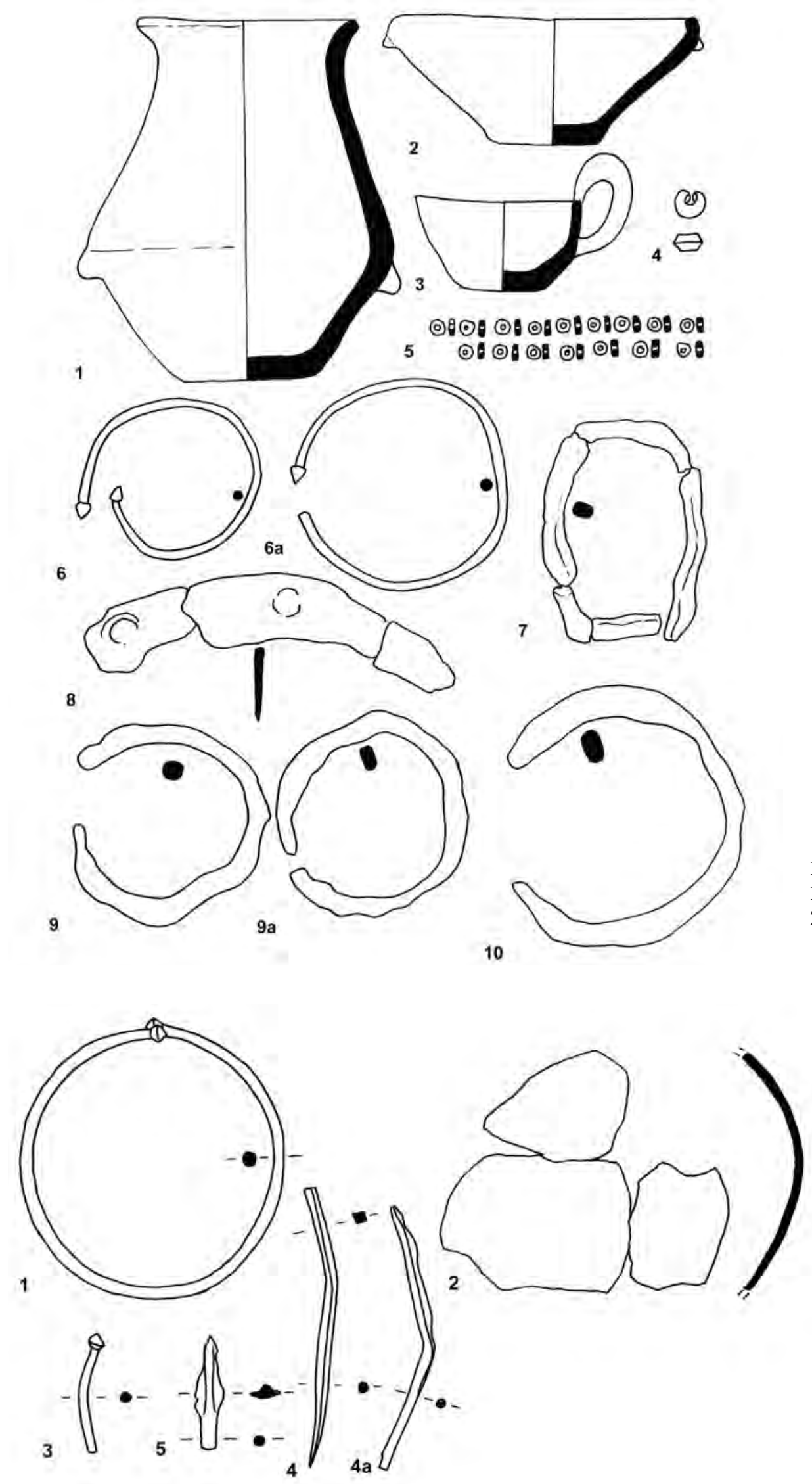

Fig. 13. Grave 8 from the Ozd-Piscul Deagului cemetery (after VAsiliev, ZRíNYI 1974).
Fig. 14. Grave 50 from the Alsótelekes-Dolinka cemetery (after Kemenczei 1994). 
two temple rings at the position of the dislocated head. ${ }^{63}$ There is also the particularly interesting double inhumation grave 6, with the burials of an adult man and woman. Under the akinake there was a temple ring. ${ }^{64}$

At the Târgu Mureș cemetery there was an inhumation grave with fragments of two temple rings with conical terminals. The important find for dating is the fibula with the foot in the shape of a Boeotian shield. ${ }^{65}$ At the Simeria (Piski) cemetery one grave contained a temple ring. ${ }^{66}$ In the Proștea Mică (Tirnăvioara) cemetery a temple ring was also found. ${ }^{67}$ The site of Saharna Mare contained three silver temple rings. It is assumed that it could have been a hoard. ${ }^{68}$ There is an interesting find of bronze rings with conical thickening from the Orosia settlement. At the bottom of the pit there was an inhumation burial of a child in a contracted position with two ceramic vessels in front. Along with the remains of the child, aged between 12 and 18 months, there were other finds, while each hand had a single bronze bracelet with overlapping terminals ending with conical thickenings, $3.7 \times 4.0$ and $3.8 \times 4.0 \mathrm{~cm}$ in diameter; the expanded part of one of them is decorated with oblique incisions combined in a braid motif. Therefore, in this cemetery this jewellery form served as a bracelet, which is confirmed by the age of the deceased, excluding the possibility of long hair with braids where they could have been worn as temple rings. Still, we do not completely rule out the possibility that they were originally made as temple rings and later put on the child's hands as bracelets. ${ }^{69}$

Bronze temple rings with conical thickening terminals have also been found at sites outside the Transylvanian region, which are marked during the Early Iron Age by the material heritage associated with other cultural groups. It is particularly interesting that this form of temple rings appears very rarely at the sites of the Ferigile group. Cremation grave 1 of tumulus 10 in the Tigveni cemetery contained a temple ring which was described as a bracelet. The find is

63 Marinescu 1984, 50 and Fig. 8B/1a-b.

64 Marinescu 1984, 50 and Fig. 11/7. - Vulpe 1990, Pl. 44A. - It should also be mentioned that grave 5 , also with a buried adult woman (Marinescu 1984, 50, 77 and Fig. 10/4. - Vulpe 1990, 128), contained a double-loop fibula with a rectangular foot with two holes dated to the late $7^{\text {th }}$ and the beginning of the $6^{\text {th }}$ century BC, also with parallels in the Balkans: Kemenczei 2004, 81 and Fig. 2/13. The grave also contained arrows and a bronze mirror: Teržan, Hellmuth, Heimann 2011, 263 and Fig. 8. - Grave 5 is considered to be a burial of a female archer warrior.

65 Kemenczei 2004, 79 and Fig. 2/1-2.

66 Kemenczei 2004, 89 and Fig. 3/13.

67 KemencZei 2004, 90 and Fig. 4/2.

68 NicUliţă et al. 2011, 193, 195-196 and Fig. 3.

69 UrSuţiU, URÁk 2016, 256, 258-259, 261 and Fig. 1/6-7, Pls. 2; $3 / 2-3$. considered to be atypical for the Ferigile group; the tumulus was dated to the first half of the $5^{\text {th }}$ century BC. ${ }^{70}$ In the Gogoșu cemetery in the Danube area, in tumulus XXVIII with a single inhumation grave, a small temple ring was found next to the head of the deceased; one of its terminals is straight, and the other ends with a conical thickening measuring only $2.6 \mathrm{~cm}$ in diameter. ${ }^{71}$ Also, east of the Carpathians, there are two temple rings from grave 1 from the Giurgiulești cemetery in Moldova. The grave and the other finds, like a fibula with the foot in the shape of a Boeotian shield, show a connection with the finds from Transylvania. ${ }^{72}$

Aside from the sites of the Ciumbrud group in Transylvania, the highest number of finds of temple rings with conical thickening terminals is currently known from sites located in northeast Hungary (Fig. 16), associated with the Vekerzug group, where they have been dated to the period from the second half of the $7^{\text {th }}$ to the middle of the $6^{\text {th }}$ century $\mathrm{BC}$, with an emphasis on the connections with Transylvania, their probable place of origin. ${ }^{73}$

The Alsótelekes-Dolinka cemetery has several graves with finds of bronze temple rings with conical thickening terminals, described as bracelets, which are associated with the graves from the older burial phase, i.e. the second half of the $7^{\text {th }}$ century BC. ${ }^{74}$ Grave 16 contained a fragment with a single preserved conical thickening at the end ${ }^{75}$ grave 33 had fragments of a temple ring: ${ }^{76}$ grave 43 included a temple ring; ${ }^{77}$ and cremation grave 50 , with the remains of a deceased woman laid inside the grave pit, contained a temple ring and a fragment with a preserved conical thickening (Fig. 14). ${ }^{78}$

70 Popescu, Vulpe 1982, 87 and Fig. 14/1. - The Tigveni cemetery is characterized by cremation graves under tumuli; the finds important for the dating are fibulae with the foot shaped like a Boeotian shield and the Marvinci-Gogoşu type.

71 Berciu, Comşa 1956, 425, 428 and Fig. 151/5. - The grave contained a fibula of the Marvinci-Gogoşu type (Fig. 151/2): Vulpe 1990, 120. - The cemetery is dated to the second half of the $7^{\text {th }}$ and the beginning of the $6^{\text {th }}$ century BC.

72 KemencZei 2004, 83.

73 PÁRducz 1965, 204-206. - Chochorowski 1985a, 61-65. Vulpe 1990. - Kemenczei 2009, 80-81. - Kozubová 2018, 31-32. - Kozubová 2019, 107-109 and Fig. 31.

74 Patay 1961, 42. - Chochorowski 1985a, 65. - Kemenczei 1994, 89. - Patay, Kiss 2002, 114-115.

75 Patay 1961, 31 and Pl. VI/5. - Chochorowski 1987, 172-173 and Fig. 6/9.

76 Patay 1961, 32 and Pl. VI/9. - Chochorowski 1987, 172-173 and Fig. 6/18. - KemenCZei 2009, 81, 162 and Pl. 134/23.

77 Patay 1961, 33 and Pl. VI/10. - Kemenczei 2009, 80, 162 and Pl. $135 / 2$.

78 Patay 1961, 34 and Pl. VI/13-14. - Chochorowski 1987, 172173 and Fig. 6/24-25. - Kemenczei 1994, 84 and Fig. 3/2-3. - KEMenCZei 2009, 163 and Pl. 135/12, 14. - Kozubová 2018, 32 and Fig. 19c. - Kozubová 2019, 107 and Fig. 30/16. 
Cremation grave $35 \mathrm{a}$ at the Tiszavasvári-Csárdapart cemetery included two bronze temple rings defined as bracelets. ${ }^{79}$ At the Dédestapolcsány-Verebce-terón cemetery, double grave 2 had two urns. One urn, containing the remains of a child aged 10 to 11 , included a bronze bracelet with overlapping terminals and two rings. The other urn contained the remains of a child aged 3.5 to 4.5 , and a bronze ring with one preserved terminal that is conically expanded. The temple ring is associated with the Pontus-Transylvanian type, dated to the second half of the $7^{\text {th }}$ and the first half of the $6^{\text {th }}$ century BC. ${ }^{80}$ The Ártánd cemetery included two temple rings that are believed to have served as earrings. They have conical thickenings, and one of them has a bronze conical pendant on the body. It is assumed that the third temple ring could have served as a bracelet and that it probably originated from the same grave. ${ }^{81}$ The Pilinyi cemetery included a bronze temple ring defined as a bracelet. ${ }^{82}$ Another find is a temple ring from the Sajógömör cemetery. ${ }^{83}$ An unknown site in the area of Zips in eastern Slovakia included a bronze temple ring with conical thickening terminals. Around the temple ring there is another smaller ring with an expansion. The temple ring is dated to the end of the $7^{\text {th }}$ and the first half of the $6^{\text {th }}$ century BC, with parallels in northeast Hungary and in the area of the Ciumbrud group. ${ }^{84}$

Somewhat different smooth rings with small conical thickenings, made of electrum and found in the Tapiószele cemetery, are assumed to have served as earrings and are attributed local origin, dated from the end of the $6^{\text {th }}$ century BC. ${ }^{85}$ Cremation grave 302 , with the remains of a woman laid directly into the grave pit, included a smooth ring with small conical thickenings. ${ }^{86}$ Two small identical rings have

79 Kemenczei 2009, 155 and Pl. 121/9.

80 Tóth 2012, 64, 74 and Pl. 5/6. - Kozubová 2018, 32 and Fig. 19a. - Kozubová 2019, 107 and Fig. 30/6.

81 Párducz 1965, 139, 204 and Pl. XXII/1-3. - Two gold rings, defined as bracelets, with terminals that have conical expansions have also been found in Ártánd. They were found in fragments and it is possible that the ends were folded. Their bodies are decorated with series of four knobs made in the granulation technique. There are also knobs at the top of the conical thickenings and in their lower part: PÁrducz 1965, 146 and Pl. XX/1-2. - Kemenczei 2009, 80, 85, 116 and Pl. 6/2-3.

82 Patay 1955, 66, 72 and Pl. XVI/10. - PÁrducz 1965, 206. - KeMENCZEI 2009, 81, 173 and Pl. 171/26.

83 Párducz 1954, 70. - Vasiliev 1970, 6. - Marinescu 1984, 80, n. 208.

84 Benediková 2007, 70 and Fig. 6/11. - Benediková 2017, 357 and Fig. $13 / 10$.

85 Párducz 1966, 85.

86 PÁrducz 1966, 61 and Pl. XXXIX/27. - Сhochorowski 1985a, 65 and Fig. 13/13. - KemenCZei 2009, 138 and Pl. 88/8.

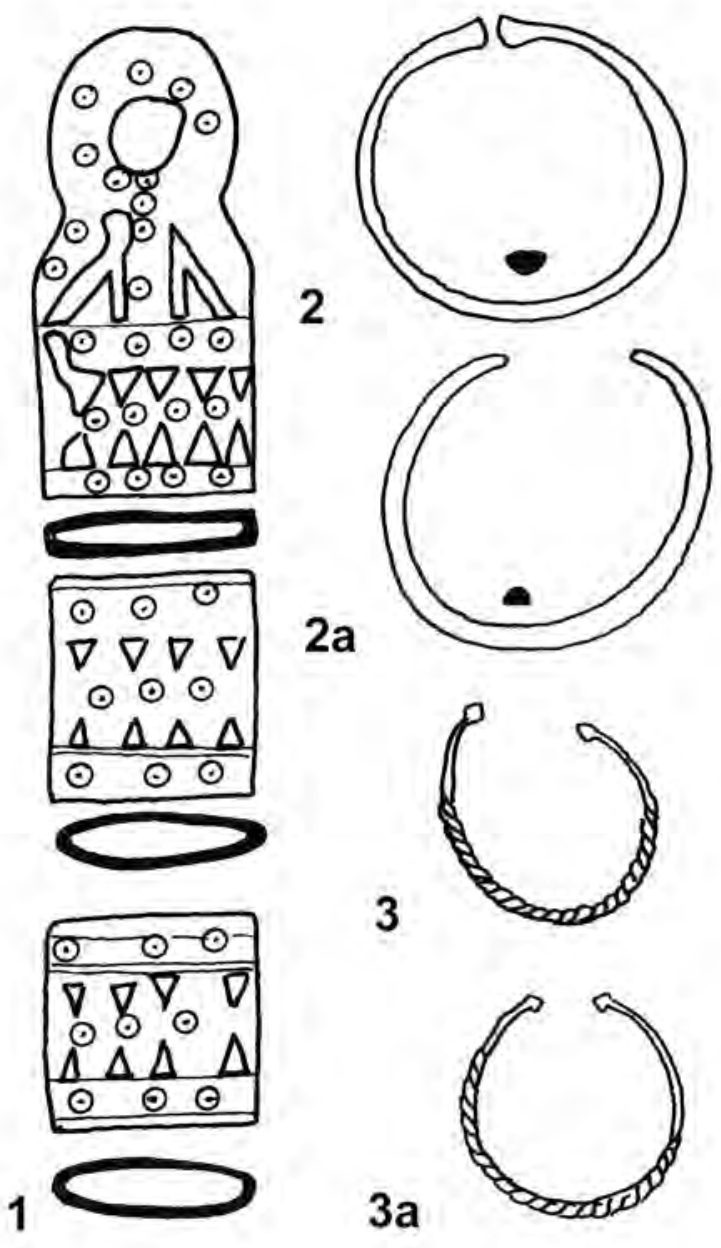

Fig. 15. The finds from Dulbok Dol (after HRistov 2004).

been found in grave $337,{ }^{87}$ while cremation grave 341 contained four entire rings and one fragmented ring, all with small conical thickenings. ${ }^{88}$ Cremation grave 392 contained two rings with small hemispherical thickenings, ${ }^{89}$ while cremation grave 462 contained a small twisted ring with a button-shaped terminal. ${ }^{90}$ Cremation grave 32 in the Tiszavasvári-Csárdapart cemetery, dated to the $6^{\text {th }}$ century BC, included a small, round-section silver ring ending with small spherical thickenings. ${ }^{91}$

As a separate variant we can single out twisted rings with expanded conical terminals found at the sites in northern Bulgaria and made of silver (Fig. 16). For instance, the

87 PÁrducz 1966, 61 and Pl. XLIX/32, 34.

88 PÁrducz 1966, 67 and Pl. L/26-31.

89 PÁrducz 1966, 73 and Pl. LXIV/11-12.

90 PÁrducz 1966, 80 and Fig. 5/11.

91 Kemenczei 2002, 45 and Fig. 11/1. - KemenCZei 2009, 81, 155 and Pl. 120/4. - Kozubová 2018, 32 and Fig. 17 b. 


\begin{tabular}{|c|c|c|c|c|c|c|c|c|c|c|c|c|}
\hline 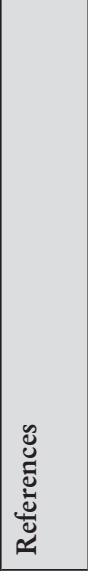 & 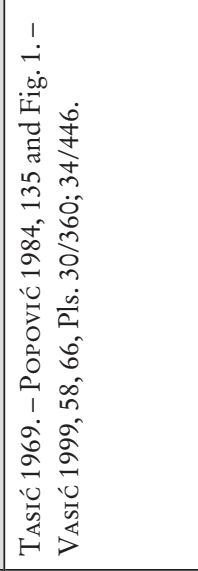 & 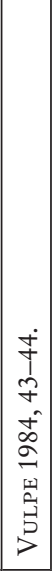 & 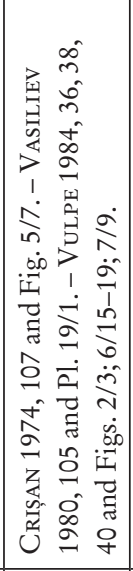 & 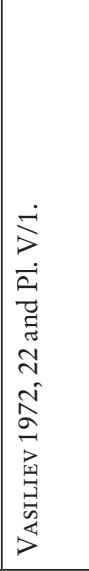 & 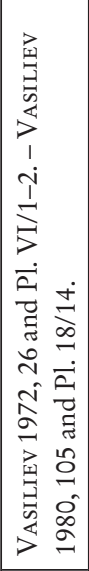 & 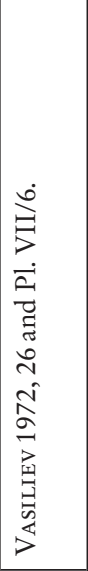 & 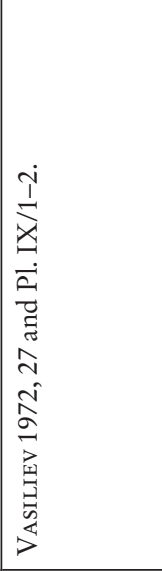 & 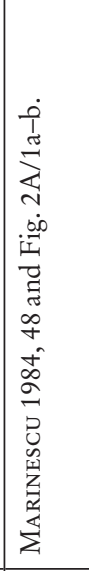 & 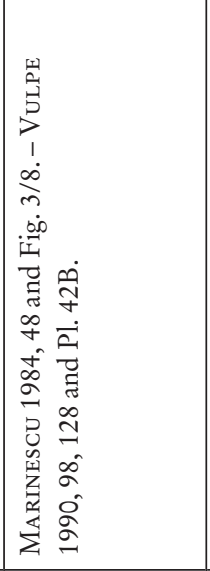 & 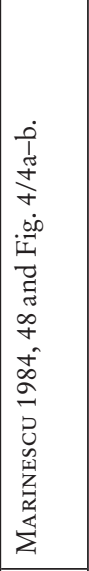 & 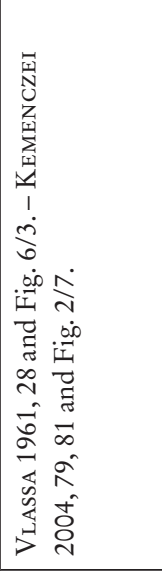 & 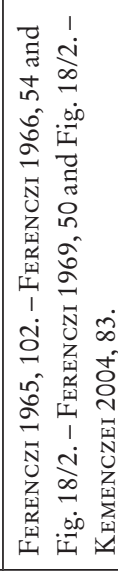 \\
\hline 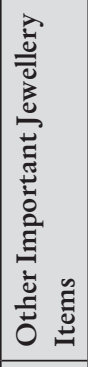 & 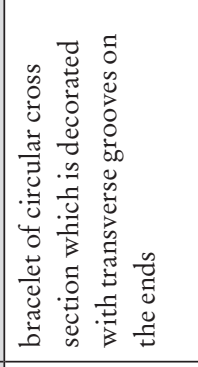 & & & 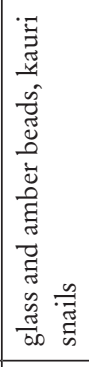 & $\begin{array}{l}0 \\
\tilde{J} \\
0 \\
0 \\
0 \\
0 \\
0 \\
0 \\
\infty\end{array}$ & 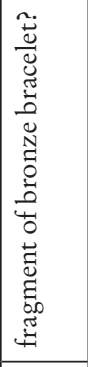 & 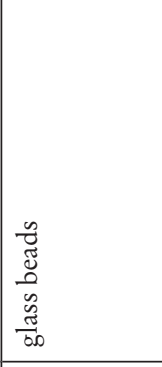 & & 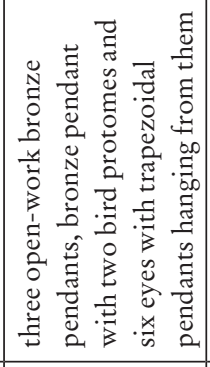 & 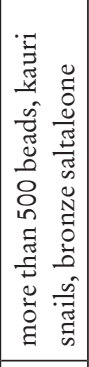 & 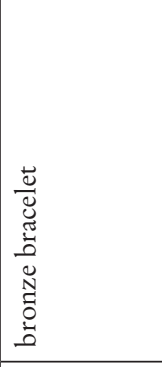 & 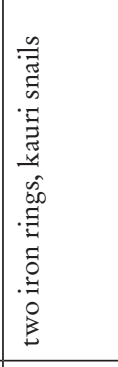 \\
\hline 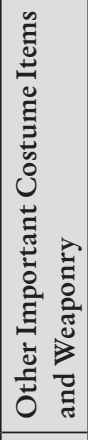 & 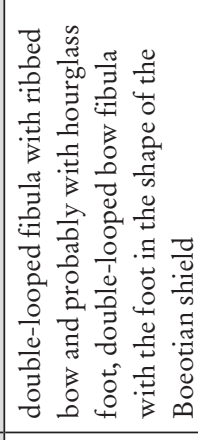 & & & & & & 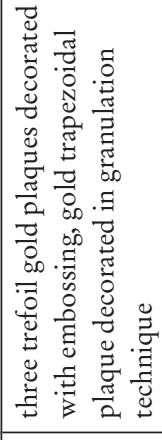 & & 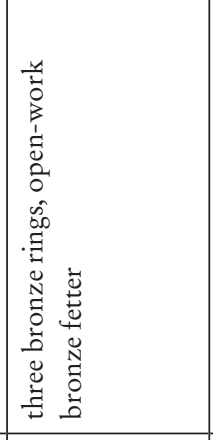 & & 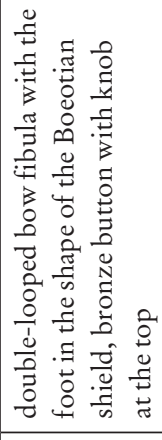 & 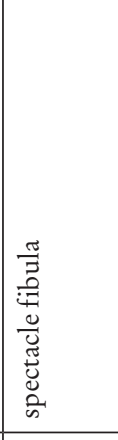 \\
\hline 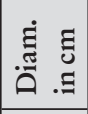 & & \begin{tabular}{|l|} 
\\
$\dot{r}$ \\
\end{tabular} & 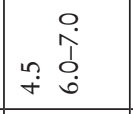 & $\stackrel{?}{+}$ & $\begin{array}{l}\text { N } \\
\text { in }\end{array}$ & 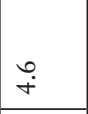 & in & $\begin{array}{lll}2 & n \\
\text { in } & \text { in }\end{array}$ & $\stackrel{\infty}{-}$ & 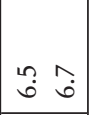 & \begin{tabular}{|l}
$-\vec{b}$ \\
$\times$ \\
$x$ \\
0 \\
0 \\
\end{tabular} & \begin{tabular}{|l} 
\\
in
\end{tabular} \\
\hline$\dot{z}$ & 4 & - & 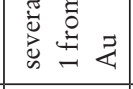 & - & \pm & - & \pm & 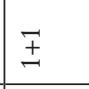 & - & \pm & - & \pm \\
\hline$\stackrel{\infty}{<}$ & & & & $\begin{array}{ll}\vec{z} & 0 \\
\bar{y} & 1 \\
\end{array}$ & 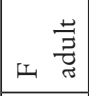 & 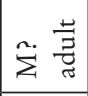 & 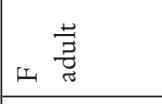 & 丰营 & 를 움 & 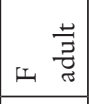 & 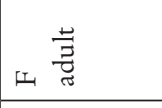 & 圷 \\
\hline 苞 & 焉 & 壳 & 焉 & 㐏 & 昰 & 昰 & 昰 & 壳 & 壳 & 顽 & 顽 & 昰 \\
\hline 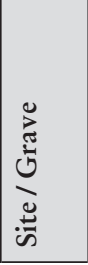 & 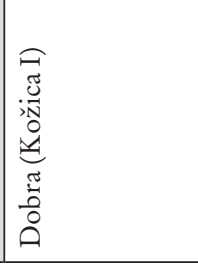 &  & 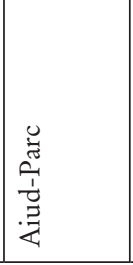 & $\overrightarrow{\vec{G}}$ & 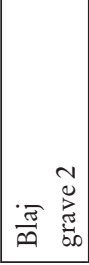 & 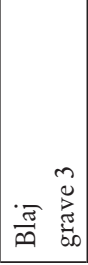 & 画总 & 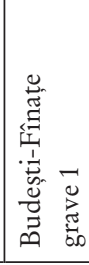 & 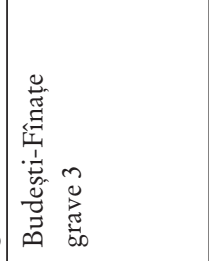 & 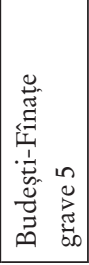 & 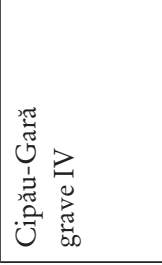 & 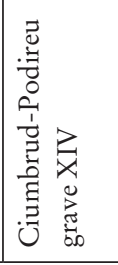 \\
\hline
\end{tabular}




\begin{tabular}{|c|c|c|c|c|c|c|c|c|c|c|c|c|c|c|c|c|}
\hline 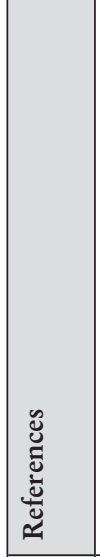 & 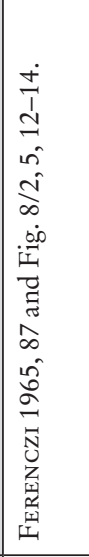 & 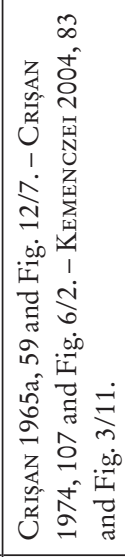 & 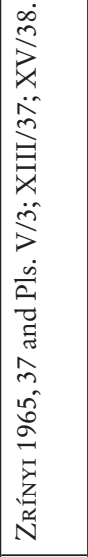 & 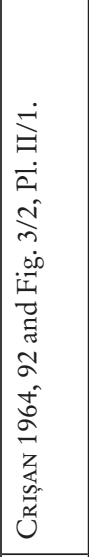 & 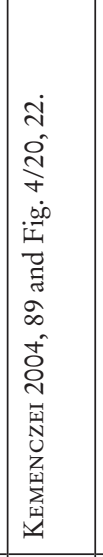 & 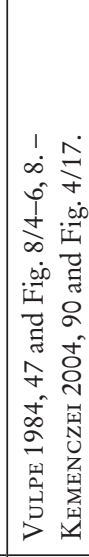 & 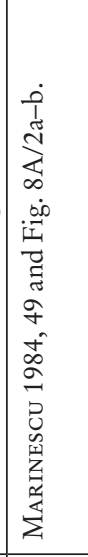 & 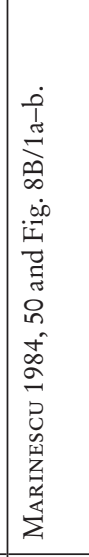 & 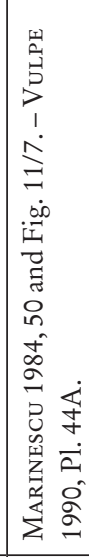 & 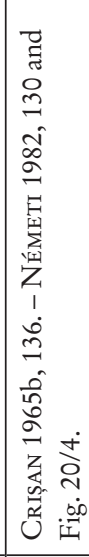 & 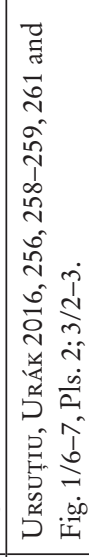 & 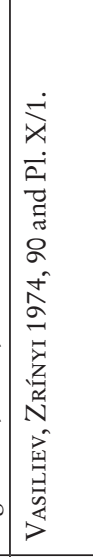 & 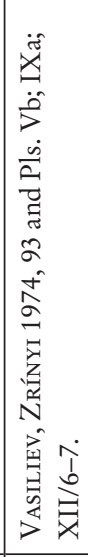 & 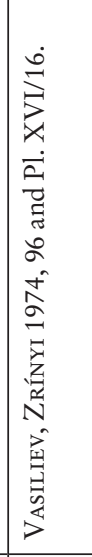 & 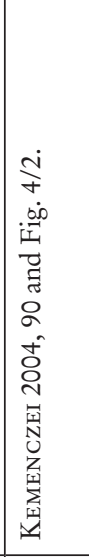 & 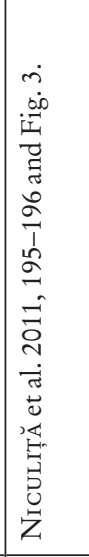 \\
\hline 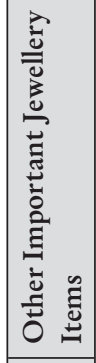 & & & & & 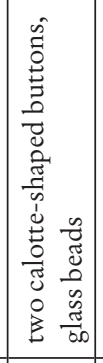 & 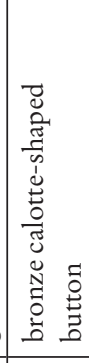 & 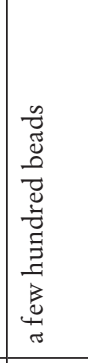 & 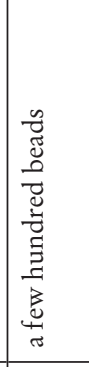 & 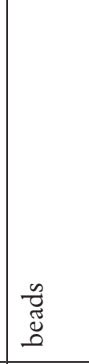 & & 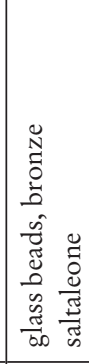 & & 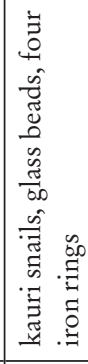 & & 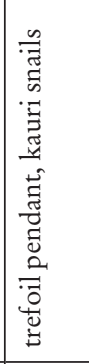 & 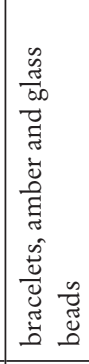 \\
\hline 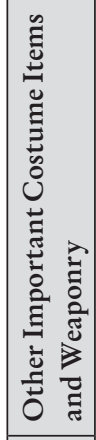 & & 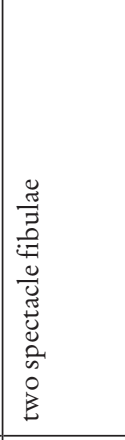 & & & 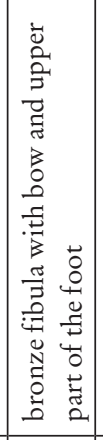 & 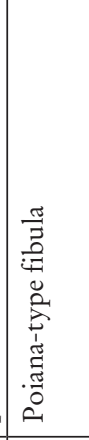 & & & & & & & & & 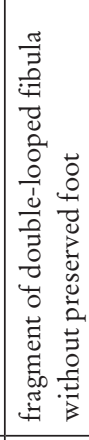 & \\
\hline 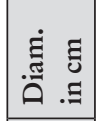 & in & o & O & & & & $\stackrel{\text { ॥̊ f }}{+}$ & $\begin{array}{ll}0 & \infty \\
\text { in } & \stackrel{0}{n} \\
\end{array}$ & in & & 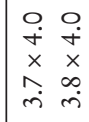 & & & & 卑 & \begin{tabular}{|l}
$\mid$ \\
$\dot{f}$ \\
$\dot{j}$ \\
$\dot{+}$
\end{tabular} \\
\hline$\dot{z}$ & 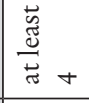 & - & \pm & & \pm & 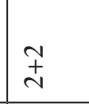 & $\exists$ & $\exists$ & - & - & \pm & - & \pm & - & - & $\underbrace{\infty 00}_{m}$ \\
\hline 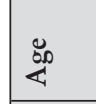 & & 屴蒙 & 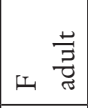 & & & & ๘ & 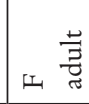 & 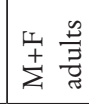 & & 긍 & & 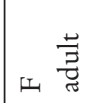 & & & \\
\hline$\stackrel{\bar{J}}{\frac{\tilde{J}}{2}}$ & 悹煦 & 壳 & 焉 & 焉 & 壳 & 壳 & 㐏 & 㐏 & 壳 & 鹿总 & 㐏 & 昰 & 壳 & 壳 & 壳 & 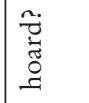 \\
\hline ड़ & 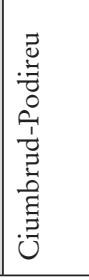 & 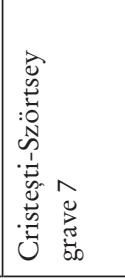 & 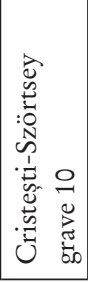 & · 콕 & 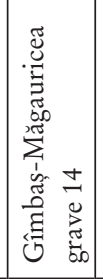 & 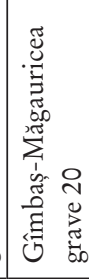 & 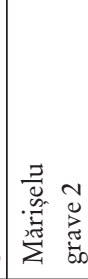 & 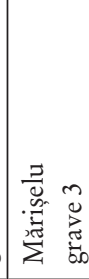 & 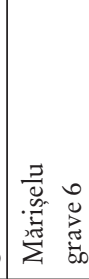 & 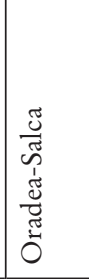 & \begin{tabular}{|l}
$\frac{\pi}{0 .}$ \\
0 \\
0 \\
0 \\
0
\end{tabular} & 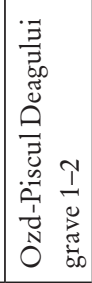 & 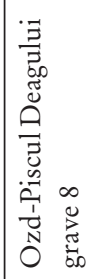 & 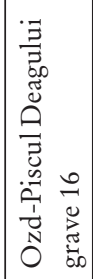 & 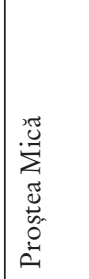 & 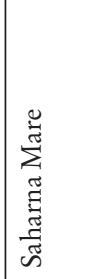 \\
\hline
\end{tabular}

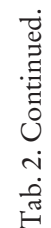




\begin{tabular}{|c|c|c|c|c|c|c|c|c|c|c|c|c|}
\hline 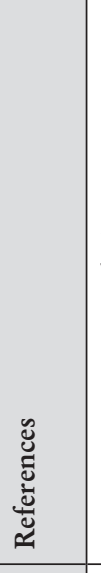 & 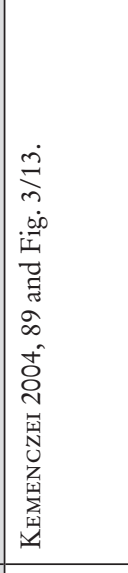 & 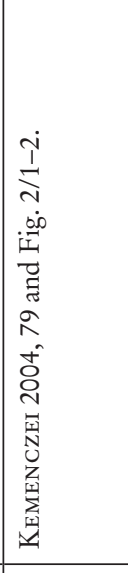 & 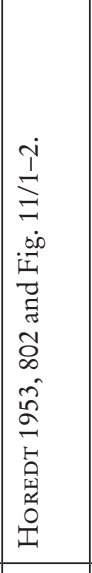 & 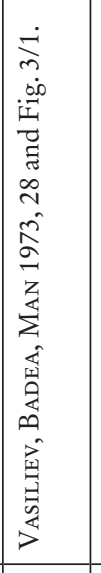 & 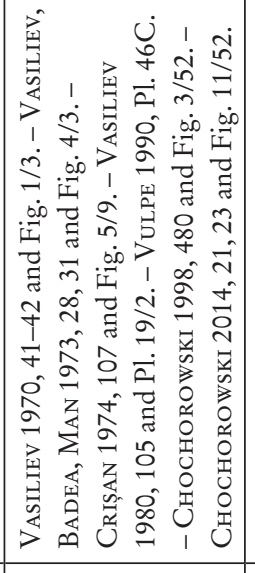 & 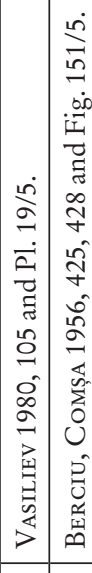 & 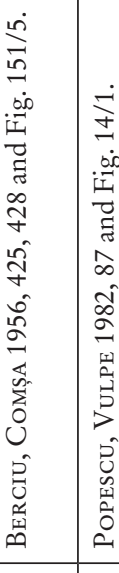 & 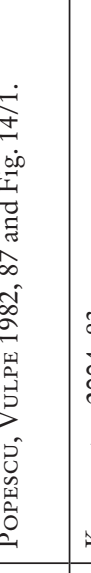 & 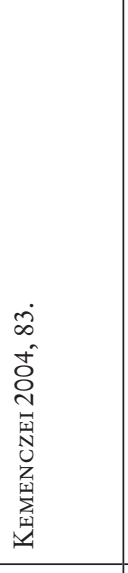 & 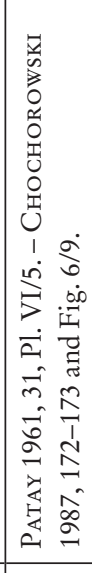 & 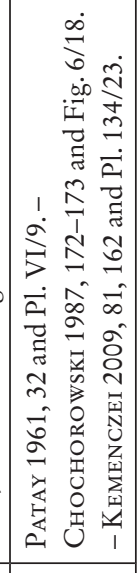 & 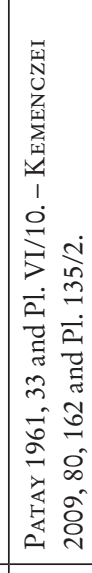 \\
\hline 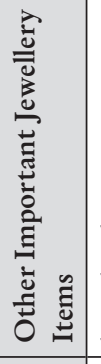 & 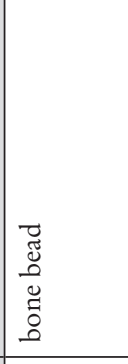 & & 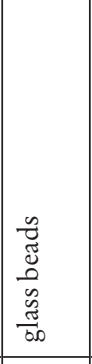 & & 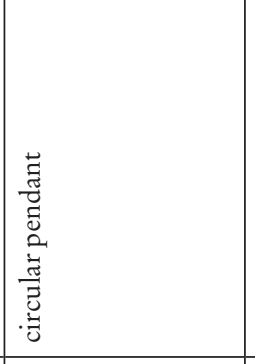 & & & & 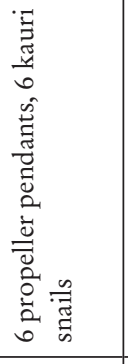 & & & \\
\hline 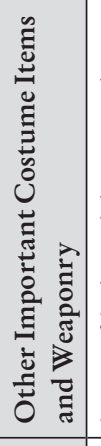 & 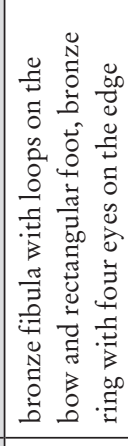 & 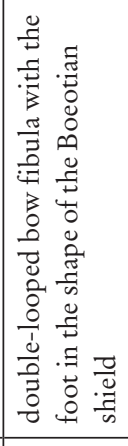 & & & 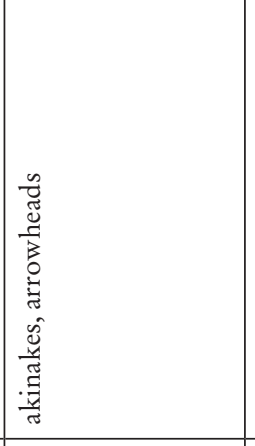 & & 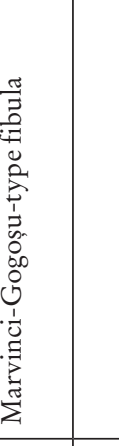 & & 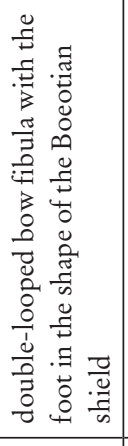 & & & \\
\hline 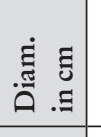 & ng & & $\begin{array}{ll} & 0 \\
i & 0 \\
\end{array}$ & 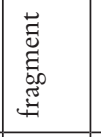 & f & & i & & & & $\begin{array}{l}0 \\
\infty_{0}\end{array}$ & \begin{tabular}{|l} 
\\
$i$
\end{tabular} \\
\hline$\dot{z}$ & - & \pm & \pm & - & 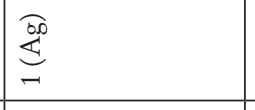 & & - & - & \pm & - & - & - \\
\hline$\frac{80}{4}$ & & & 地 & 为 & 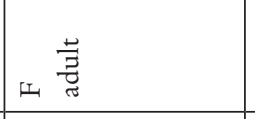 & & & & & & & \\
\hline 造 & $\underline{\Xi}$ & $\underline{\Xi}$ & $\underline{\Xi}$ & 宓 & 吾 & & 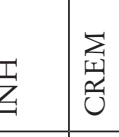 & 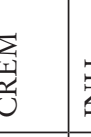 & 壳 & 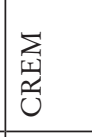 & 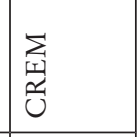 & 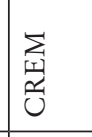 \\
\hline हूँ & 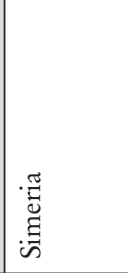 & 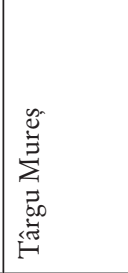 & 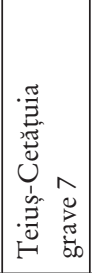 & 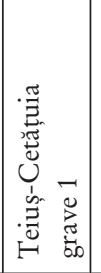 & 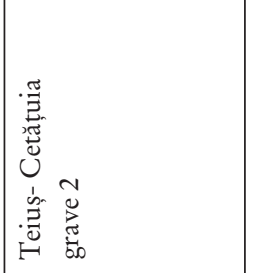 & 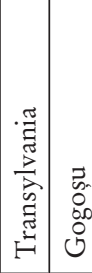 & 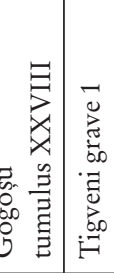 & 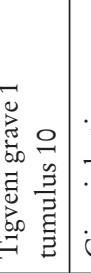 & 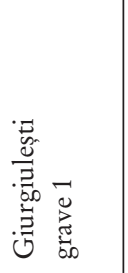 & 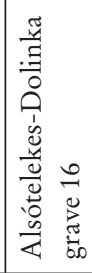 & 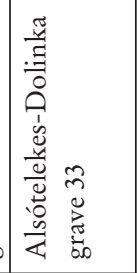 & 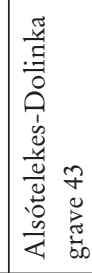 \\
\hline
\end{tabular}

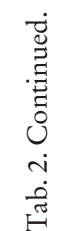




\begin{tabular}{|c|c|c|c|c|c|c|c|c|c|c|c|c|}
\hline 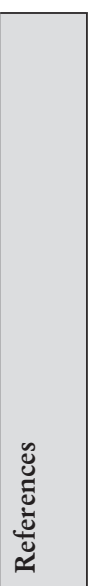 & 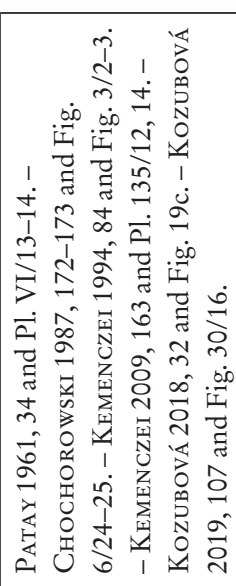 & 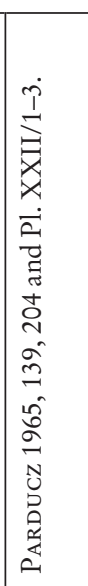 & 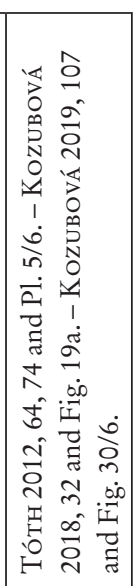 & 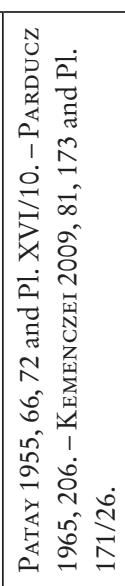 & 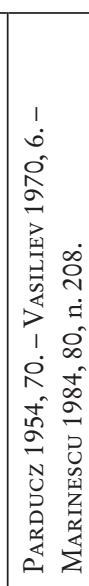 & 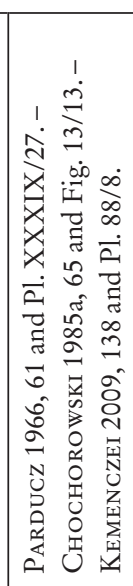 & 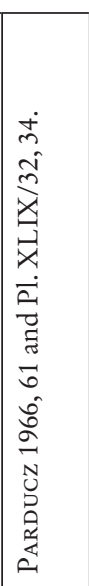 & 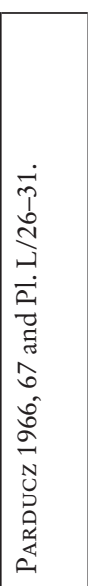 & 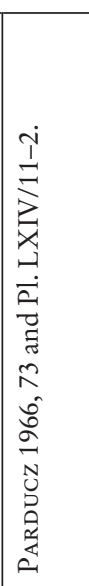 & 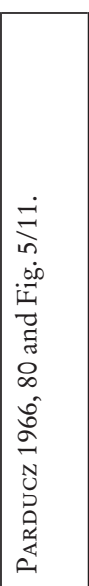 &  & 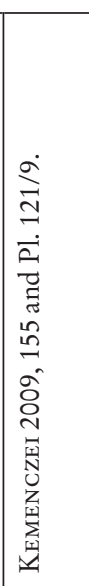 \\
\hline 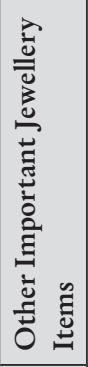 & & & 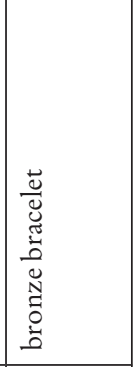 & & & & & & & & & \\
\hline 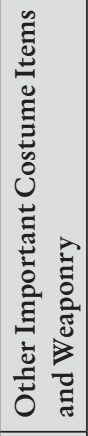 & 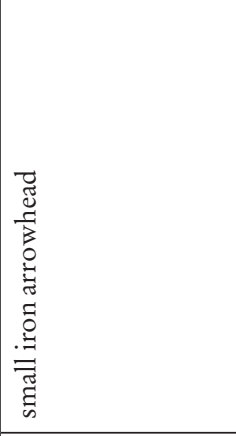 & & & & & & & & & & 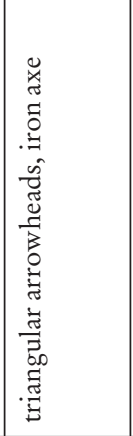 & \\
\hline 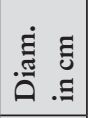 & ن. & $\underset{f}{\rightarrow} \stackrel{\infty}{i n}$ & ir & $\stackrel{+}{\sigma}$ & & & & & & & $\mid \begin{array}{c}\hat{i} \\
\dot{x} \\
\overrightarrow{+}\end{array}$ & $\mid \begin{array}{l}+ \\
\dot{\theta}\end{array}$ \\
\hline$\dot{z}$ & $\alpha$ & $\mathrm{m}$ & - & - & - & - & 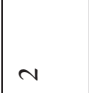 & in & 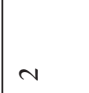 & - & $\underset{-}{\stackrel{B 00}{\leftrightarrows}}$ & 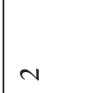 \\
\hline 总 & & & 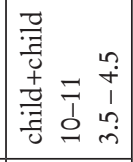 & & & 出 & & & & & & \\
\hline 苛 & 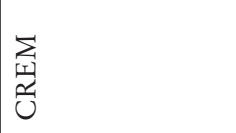 & 局 & 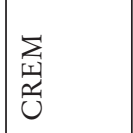 & 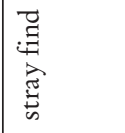 & 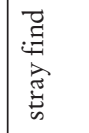 & 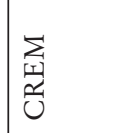 & 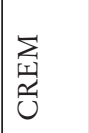 & 童 & 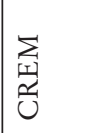 & 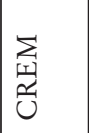 & 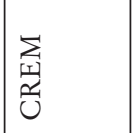 & 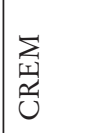 \\
\hline 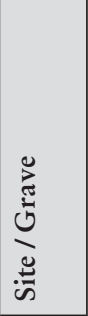 & 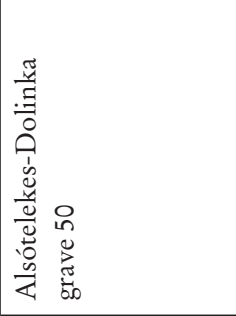 & 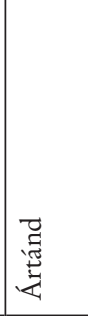 & 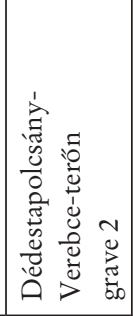 & 点 & 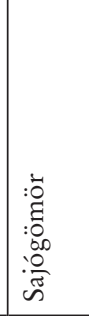 & 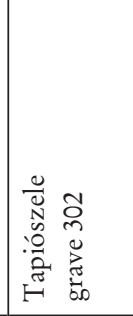 & 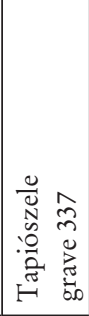 & 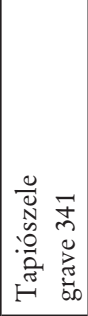 & 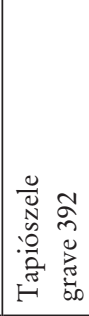 & 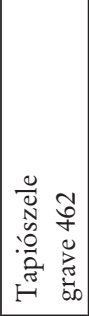 & 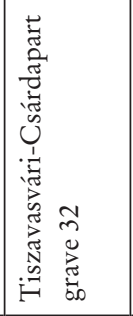 & 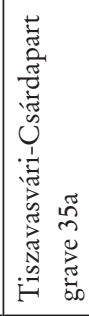 \\
\hline
\end{tabular}

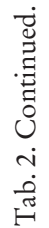


Malka Brestnica site included a twisted temple ring with smooth overlapping terminals ending with conical thickenings. ${ }^{92}$ A tumulus at the Dulbok Dol site, which seems to be a rich burial of a woman, included two twisted temple rings with conical terminals; the part before the terminals is also smooth (Fig. 15). They are 4.3 and $4.8 \mathrm{~cm}$ in diameter; the grave has been dated to the $7^{\text {th }}$ century BC. ${ }^{93}$ The same dating applies to the tumuli from the Radjuvene site, where as many as eight more or less twisted temple rings have been found. ${ }^{94}$ This ensemble of finds is similar to the contemporary one in Donja Dolina. We must also mention the finds from grave 1 of tumulus 2 at the Kočan site, where five small silver rings have been found; four of them are twisted, but only in the middle part, while the terminals are smooth. The rings have a small diameter: 1.5 to $2.6 \mathrm{~cm}$. The grave has been dated to the second half of the $6^{\text {th }}$ century BC. ${ }^{95}$

On the other hand, what mostly appears in the Vekerzug group cemeteries are differently shaped small rings, made of bronze, silver, electrum, or gold; they are assumed to be hair decorations, while the first publications listed them as earrings. The terminals can have variously shaped thickenings (i.e. conical, mushroom-shaped, plate). Most finds come from cremation graves, but the finds on both sides of the head in inhumation graves show that they were head/hair decorations. It was the characteristic head decoration of the Vekerzug group over the entire duration of the group (from the second half of the $7^{\text {th }}$ to the end of the $4^{\text {th }}$ century BC), which most often appears in female graves, as the deceased probably came from richer families. Different types have been classified considering the form of the body, with certain forms being widespread in the northeast Carpathian Basin and considered to be a product of local workshops under eastern influences. ${ }^{96} \mathrm{~A}$ particularly important insight is that those head/hair decorations were most often worn by adult women, while pairing with other forms used as body decoration makes it possible to identify several types

92 DUšEK 1964b, 69 and Pl. III/2. - DUŠEK 1974, 371 and Pl. III/15. 93 Hristov 2004, 44, 54, 59 and Figs. 1, 6-7.

94 Hristov 2004, 51, 55 and Fig. 12; 61 nos. 29-32.

95 Gergova 1987, 73 and Pl. 35/A156-159.

96 On the classification of hair ornaments at the Vekerzug group cemeteries: DušEK 1966, 34-35. - СHосHоRowsKi 1985a, 61, 64. KemencZei 2002, 29, 57-70. - KemencZei 2009, 71, 80-85. - KeMeNCZeI 2010, 118 and Fig. 10. - Ilon 2017. For the most detailed typo-chronological classification of the head/hair ornaments, see Kozubová 2013, 26-34. - Kozubová 2018, 13-55 and Fig. 10. Kozubová 2019, 106-109 and Figs. 28, 31. - Kozubová divides them into three basic types, and the Vekerzug group is particularly characterized by the second type, appearing in the first half of the $6^{\text {th }}$ century BC. of female costume, which probably indicates social stratification in the communities. ${ }^{97}$

Especially interesting finds are twisted rings made of silver, sometimes bronze, which are known in large numbers from the Szentes-Vekerzug cemetery, where they have been found both in cremation and inhumation graves and are sometimes described as earrings, with the mentioned parallels at the sites in Transylvania and northern Bulgaria, but also Donja Dolina, where, however, they have a smooth body. For the rings with conical thickening terminals there are particularly notable parallels at the Transylvanian sites, where they could have originated, but twisted bodies have not been found there, so the origin of twisting is looked for in eastern Hungary or northern Bulgaria. ${ }^{98}$ Ring terminals often have conical or spherical thickenings preceded by a short and smooth part. ${ }^{99}$ For instance, cremation grave 27 included two twisted rings with smooth and overlapping terminals, ${ }^{100}$ while cremation grave 34 included one gold and one silver twisted ring. ${ }^{101}$ Next to the head of the deceased woman in inhumation grave 46 there were two silver twisted rings described as earrings. It seems that one terminal has a conical thickening. ${ }^{102}$ The richly furnished cremation grave 61 contained rings of twisted silver wire that seemingly had a conical thickening at one end and a spherical thickening at the other, with parallels from Ártánd, dated before the middle of the $6^{\text {th }}$ century BC. ${ }^{103}$ Cremation grave 74 contained three silver twisted rings of different diameters, with

97 Kozubová 2018, 13, 39-55. - Kozubová 2019, 146-149. - Emphasis is placed on the correlation between different combinations of jewellery with respect to the gender, age, and social status of the deceased.

98 PÁrducz 1954, 35, 52, 64-65. - PÁrducz 1955, Pls. V/12; VI/4. - PÁrducz 1965, 204-205. - The listed parallels with Donja Dolina are the finds of pendants, plate fibulae, Novi Pazar type fibulae etc. (PÁRducz 1965, Pls. VIII/1; XV/11; XXII/1-2). - Interestingly, KEMENCZEI 2009, 80, mentions twisted silver temple rings with conical terminals from the graves in Donja Dolina, which are actually not present at that cemetery.

99 KemenCZEi 2009, 80. - As hair ornaments of the first type in the graves dated to the $7^{\text {th }}$ and $6^{\text {th }}$ centuries BC: Kozubová 2018, 32. Kozubová 2019, 107-108 points out that the twisted rings found at the cemeteries in eastern Hungary have a local origin and do not appear before the $6^{\text {th }}$ century BC, and are still worn in the first half of the $5^{\text {th }}$ century BC, maybe with an origin in the west, where they appear earlier (Ha C2/D1).

100 PÁrducz 1954, 30 and Pl. VI/12. - Kemenczei 2002, 31 and Fig. 4/11. - Kemenczei 2009, 80, 135 and Pl. 69/11. - Kozubová 2018, 32 and Fig. 17a.

101 PÁrducz 1954, 32 and Pl. VI/17-18. - KemencZei 2009, 80, 135 and Pl. 70/14, 17.

102 PÁrducz 1954, 34 and Pl. XIII/17-18.

103 PÁrducz 1954, 36 and Pl. XVII/8-9. - Kemenczei 2004, 87, 89 and Fig. 6/10-11. - Kemenczei 2009, 80, 135 and Pl. 74/6-7. 


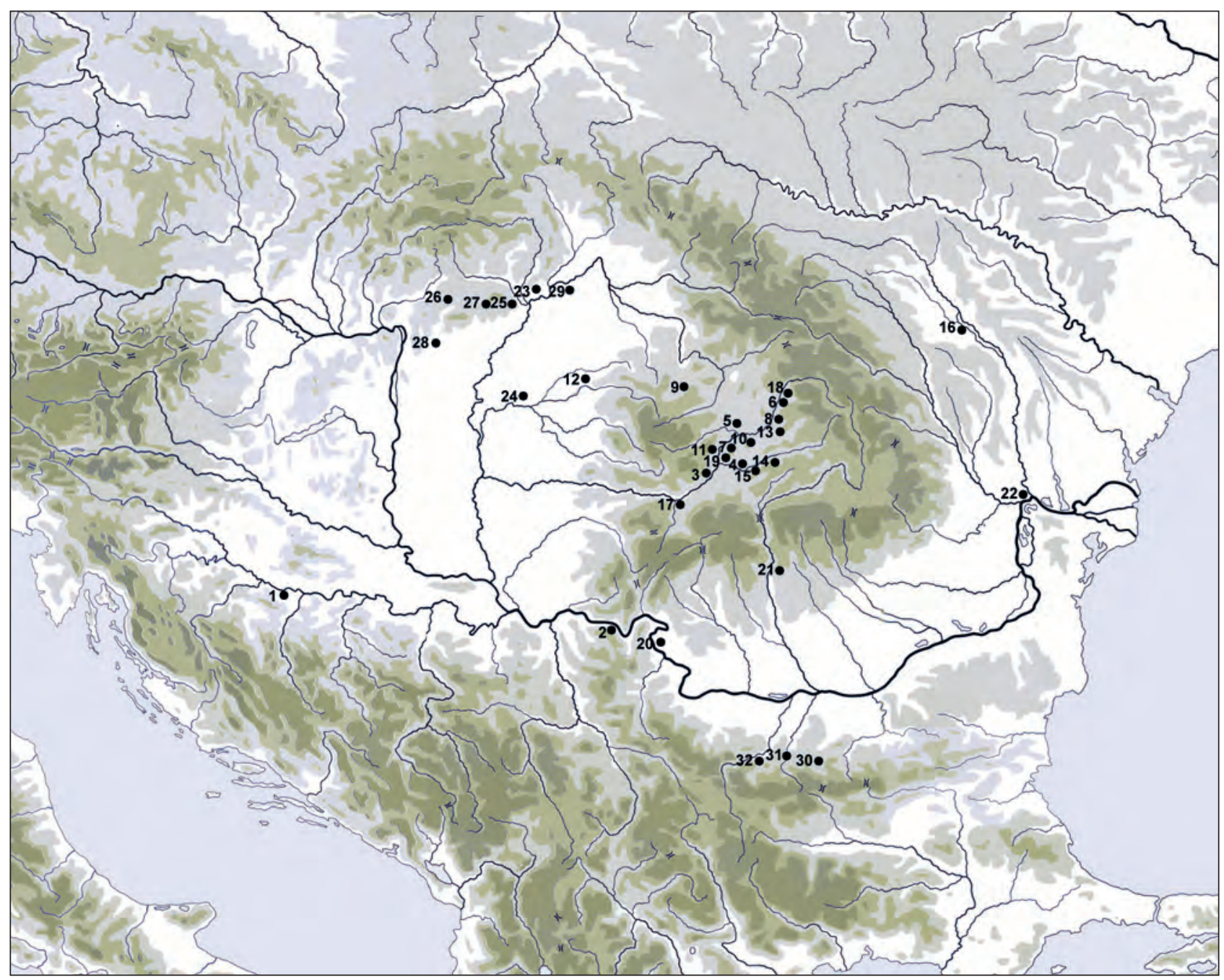

Fig. 16. Distribution map of the finds of type A temple rings, the Ciumbrud type. - 1. Donja Dolina. - 2. Dobra-Kožica I. - 3. Aiud-Gerepen and Parc. - 4. Blaj. - 5. Budeşti-Fînațe. - 6. Cipău-Gară. - 7. Ciumbrud-Podireu. - 8. Cristești-Szörtsey. - 9. Dezmir. - 10. Gîmbaş-Măgauricea. -11. Mărişelu. - 12. Oradea-Salca. - 13. Orosia. - 14. Ozd-Piscul Deagului. - 15. Proştea Mică. - 16. Saharna Mare. - 17. Simeria. - 18. Târgu Mureș. - 19. Teiuș-Cetățuia. - 20. Gogoșu. - 21. Tigveni. - 22. Giurgiulești. - 23. Alsótelekes-Dolinka. - 24. Ártánd. - 25. DédestapolcsányVerebce-terőn. - 26. Pilinyi. - 27. Sajógömör. - 28. Tapiószele. -29. Tiszavasvári-Csárdapart. - Bulgarian variant: 30. Dulbok Dol. - 31. Malka Brestnica. - 32. Radjuvene (M. Dizdar and A. Kapuran).

small conical thickenings that are pulled through each other. ${ }^{104}$ Inhumation grave 76 contained a pair of silver twisted rings with expanded conical terminals. ${ }^{105}$ On each side of the head of the deceased in inhumation grave 114 there was an electrum ring with a twisted body and conical thickening terminals. ${ }^{106}$

After listing all the known sites with the finds of temple rings with conical thickening terminals of type A or the

104 Párducz 1954, 38 and Pl. XXIV/1.

105 PÁrducz 1955, 39 and Pl. XXIV/12-13. - Сносноrowski 1985a, 136 and Fig. 47/16. - Kemenczei 2009, 136 and Pl. 75/22. 106 PÁrducz 1955, 3 and Pl. III/16-17. - Сhochorowski 1985a, Fig. 13/14. - Сносновошsкi 1985b, 227 and Pl. 4/17.
Ciumbrud type, especially in the case of closed grave assemblages from Donja Dolina (Tab. 1) all the way to the cemeteries of Transylvania and northeast Hungary (Tab. 2), it is necessary to briefly present the history of research of this very characteristic item of female jewellery, which often appears in the cemeteries of the eponymous Ciumbrud group, but also in northeast Hungary and in the Donja Dolina cemetery (Fig. 16).

Temple rings with conical terminals-the Ciumbrud type - were considered characteristic for the 'Scythian' graves in Transylvania by the first researchers, Nicolae Vlassa ${ }^{107}$ and

107 VLASSA 1961, 40. 
Ion Horațiu Crișan, who pointed out that their origin was the area along the northern shores of the Black Sea. ${ }^{108}$ This opinion on the Scythian origin was taken up by Mikulás Dušek. ${ }^{109}$ Ștefan Ferenczi also writes that smooth temple rings with conical terminals often appeared in cemeteries in Transylvania, noticeably differing from the finds from the cemeteries along the Tisza River and in southern Slovakia. Also, he mentions that this form of temple ring appears at the sites along the northern shores of the Black Sea, most often exceeding $5.0 \mathrm{~cm}$ in diameter. ${ }^{110}$ Valentin Vasiliev, in his analysis of the Blaj cemetery and in some later papers, sought the origins of temple rings north of the Black Sea, and considers their typology, distribution, origin, and the chronological framework of different forms of temple rings. In that respect, he identifies three basic types of temple rings with defined distribution areas; the first one is characterized by conical thickening terminals and is considered characteristic for the 'Scythian' group of the $6^{\text {th }}$ century BC, located north of the Black Sea, and for the sites in Transylvania and some in northeast Hungary, while they are rare in the west and south. ${ }^{111}$ A similar opinion is given by Vasiliev and Andrei Zrínyi in the analysis of the Ozd-Piscul Deagului cemetery; they identify two basic types. The first type includes temple

108 Crişan 1964, 92. - CrişAn 1974, 107 and Fig. 5/7-9. - Mention is also made of finds from the Dacian sites, which are much younger, e.g. grave dating in the $3^{\text {rd }}$ century BC from the Murighiol cemetery: Bujor 1958, 135 and Fig. 7/15-17.

109 DušEK 1964a, 284-285 and Fig. 5/9. - DušEK 1974, 371. - A similar opinion is provided by СНосновошsк 1985b, 227, who believes that the appearance of the Transylvanian group at the beginning of the $6^{\text {th }}$ century BC is related to the Scythian incursions from the east. On a reverse direction of spreading: PÁRDUCZ 1965, 206.

110 Ferenczi 1969, 49-50 and Fig. 8/2, 5, 12-14.

111 Vasiliev 1970, 41-50 and Fig. 7. - Vasiliev 1972, 48-50. Vasiliev, BadeA, Man 1973, 31. - VAsiliev 1980, 105 and Pls. 18/4; 19/1-3, 5. - NÉmETi 1982, 123. - The first variant is characterized by conical thickenings at the terminals - it is the Pontic-Transylvanian variant. The second variant is called Szentes-Vekerzug-Chotín and is widespread in eastern Hungary and Slovakia in the $6^{\text {th }}$ century BC. The last is the Pontic-South Danube variant, occurring at sites along the northern shores of the Black Sea in the $6^{\text {th }}$ century BC, and present in Thracia in the $5^{\text {th }}$ century BC. Finally, he quotes silver finds already belonging to the Late Iron Age and even to antiquity, of smaller dimensions, with larger and smaller conical expansion or one terminal ending with a smaller conical thickening, while the other just tapers. These younger forms, often described as earrings, have been found at numerous sites dating from the end of the $4^{\text {th }}$ century $\mathrm{BC}$ right up until the $1^{\text {st }}$ century AD: Rustoiu 1996, 102-103, 196-197 and Fig. 50, Pl. 51/1-10. - Rustoiv 2002, 104 and Fig. 70/23-29. - SPÂNU 2012, 52 and Fig. 9. - We also mention a silver earring with a diameter of $5.5 \mathrm{~cm}$ from the site of Šetonje-Vasina glavica (or Salakovac-Mogila-Most) in northern Serbia, which has a larger conical thickening at one terminal and a smaller one at the other, and is dated to Iron Age III (STOJIĆ, JaCANOvić 2008, 281, 283 and Figs. 131, 150, Pl. CXX/15). rings with conical thickening terminals that are believed to be characteristic for 'Scythian' cemeteries and considered as an influence from the areas north of the Black Sea. The second type is represented by two variants: the first includes temple rings from child grave 6 , of round cross section and smaller dimensions; the second includes the finds from grave 9, which have a semicircular cross section and overlapping tapering terminals, which is a characteristic of recognized type C1 in Donja Dolina. After all, temple rings are considered to be characteristic of the Ciumbrud group, distributed across Transylvania, where they came from the areas north of the Black Sea, and those in the Ozd cemetery have been dated to the beginning of the $6^{\text {th }}$ century BC. ${ }^{112}$ The same opinion is provided by Vulpe, who identifies them as one of the distinctive forms of the Ciumbrud group at the sites in Transylvania, primarily in the cemeteries; he describes them as earrings. The beginning of the group has been dated to the middle of the $7^{\text {th }}$ century BC, with three phases. ${ }^{113}$ Interestingly, when Vasić mentions the finds of double-loop fibulae with the foot in the shape of a Boeotian shield or some other forms from the Transylvanian sites (Cipău-Gară, Târgu Mureș), associating them with the finds from the western and central Balkans, he also mentions the finds of hair rings with conical terminals that are frequent at the Transylvanian sites and can be found in Donja Dolina too. ${ }^{114}$ Teržan believes that smooth wire temple rings with conical thickening terminals are one of the characteristic forms of the Vekerzug group; the elements of particular importance for dating are the finds from the graves in Donja Dolina, where they were together with various costume and jewellery items from the second half of the $7^{\text {th }}$ century BC. ${ }^{115}$

112 VAsiliev, ZRínyi 1974, 112-113, 117. Type 1: Pls. X/1; XII/6-7; XVI/16. Type 2: Pls. XI/10-11; XIII/16-17. - In the richly equipped grave 6, where a 6-year-old girl was buried: VAsiLIEv, ZRíNYI 1974, 92 and Pls. IVb; XI/10-11. - In grave 9, where an adult woman was buried, on each side of her head there are temple rings of semicircular cross section, with folded tapering terminals that are $5.8 \mathrm{~cm}$ in diameter: VAsiliev, ZRínYi 1974, 94 and Pls. VIa; IXb; XIII/16-17.

113 Vulpe 1970, 153 and Fig. 9/4. - Vulpe 1990, 127-130. - KeMENCZei 1994, 83. - Kemenczei 2002, 63. - Ciumbrud group cemeteries are composed of smaller groups of graves and date from the mid- $7^{\text {th }}$ century BC. - С HосHOROwsKi 1998, 479-480, associates the Transylvanian group with the oldest Scythian elements and also dates them around the mid- $7^{\text {th }}$ century BC, even though it is not excluded that the first Scythian influences date from the turn of the $8^{\text {th }}$ and the $7^{\text {th }}$ centuries BC. Similar to Vulpe, he concludes that the group certainly existed as early as the second half of the $7^{\text {th }}$ century BC.

114 VAsIĆ 1974, 37, n. 17. - VAsić 1987b, 545. - VAsIĆ 1999, 58, 66. KemenCZei 2009, 81.

115 TeRŽAn 1998, 515. - Contacts are also indicated by the finds of plate fibulae. 
Very significant insights were gained through the analysis of the cemeteries of Budești-Fînațe and Mărișelu, where George Marinescu noticed that temple rings with conical thickening terminals were found on both sides of the head, next to the ear opening. On the basis of the position, he concludes that they could have been worn as earrings or temple rings, both possibilities being likely, but he favours the earrings explanation, since the conical terminal for pulling through the ear is small or missing in most specimens. Also, after remarking that they differ in both size and weight, the cause is found in the age of the person that wore them. It is believed they are characteristic of female graves but can sometimes be found in male graves, taking as an example grave 2 from the Teius cemetery. They frequently appear in the Transylvanian cemeteries, where they have been dated to the second half of the $7^{\text {th }}$ century BC and later (Budești, Targu Mureș), and they appear later and more rarely among the Scythians. Instead of the previous theory of the Scythian origin of this type of temple ring, this presents them as a local form that probably originated in Transylvania. It is also pointed out that they occasionally appear in Thracia, and they are also known from the Donja Dolina cemetery, where they are contemporary with the Transylvanian finds. ${ }^{116}$ The connection of the Transylvanian cemeteries with that in Donja Dolina, based on the form of bronze temple rings with conical thickening terminals, but also some other forms of fibulae, is also recognized by Tibor Kemenczei. He dates them to the Scythian horizon and later, not excluding the possibility that they came to Transylvania from the east. In Donja Dolina, he dates them to the second half of the $7^{\text {th }}$ century BC. ${ }^{117}$ There is a particularly interesting interpretation of a find from a child grave in the Orosia settlement where rings with conical terminals were documented as serving as bracelets, while allowing for the possibility that they were originally made as temple rings. This form is considered to be characteristic for the Ciumbrud group. On the basis of specific contexts, it can be concluded that they were worn as hair decorations, mostly by adult women, and were dated to the $6^{\text {th }}$ and $5^{\text {th }}$ centuries BC. ${ }^{118}$

There are particularly important studies of the hair decorations of the Vekerzug group by Anita Kozubová, whose

116 Marinescu 1984, 80-81. - Some graves have a single temple ring, and some have two of them - one on each side. To the middle and the second half of the $7^{\text {th }}$ century BC can be dated finds from cemeteries of Budeşti, Cipău, Târgu Mureş, Aiud, and Cristeşti II, and the older phase of the Ferigile (south) cemetery. Younger cemeteries are Mărişelu, Ciumbrud, Blaj, Ozd, and Ferigile north.

117 Kemenczei 1988, 93. - Kemenczei 2002, 66. - Kemenczei 2004, 81, 83, 90. - Kemenczei 2009, 80-81. - The connection of Transylvania and the eastern Carpathian Basin with the northern Balkans is also indicated by some types of bronze fibulae.

118 URsuţiU, URÁK 2016, 261, 264-265. considerations include the finds of temple rings with conical thickening terminals, which she defines as the Ciumbrud type, including the rare occasions when they are worn as bracelets. As opposed to the characteristic forms of the Vekerzug group, they are much larger in diameter, with a smooth body and terminals that are spaced, touching, or overlapping. The Ciumbrud-type temple rings are distributed in four areas - on the territory of the eponymous Ciumbrud group in Transylvania, at the Vekerzug group sites, in Donja Dolina, and in the area along the northern shores of the Black Sea. The Ciumbrud group is characterized by the smooth body, which is sometimes made of precious metals. Their wearing period is the second half of the $7^{\text {th }}$ and during the first half of the $6^{\text {th }}$ century BC, which is also the dating of the finds from the Donja Dolina cemetery, where they were worn in a somewhat different way. At the sites located in the area along the northern shores of the Black Sea, they appeared later, from the $6^{\text {th }}$ to the $3^{\text {rd }}$ century BC, and they were worn as bracelets, which gives chronological priority to the finds from the distribution area of the Ciumbrud group and Donja Dolina. ${ }^{119}$

\subsection{Type B: Temple Rings with Decorated Terminals - the Donja Dolina Type}

The temple rings classified as type B at the cemetery in Donja Dolina are bronze temple rings of rounded cross section, with terminals decorated with a group of transverse grooves, while the rest of the body is smooth. The terminal itself is either straight or conical (Fig. 1), and they can be slightly spaced or slightly overlapping. This type of temple ring has been found only in Donja Dolina, with no known finds at other sites so far. Most of them have been found in inhumation graves of adult women on both sides of the head of the deceased - a total of six; the only cremation grave is grave S. Jakarić 14 (Fig. 8). A type B temple ring which probably comes from a destroyed grave, $7.6 \mathrm{~cm}$ in diameter, has been dated to phase IIa after Marić, and continues into phase IIb. ${ }^{120}$

It is extremely rare for a type $\mathrm{B}$ temple ring to be the only form in a grave; as a rule, they are accompanied by type A1 or type C1 (Tab. 3). In two graves they are combined with types A1 and C1 (Figs. 3-4), and it seems that grave M. Petrović Jr. 6 also includes a type D2 (Tab. 3). Type $\mathrm{B}$ temple rings are worn in an interesting way. When they appear as the only form in the grave, there are four

119 Kozubová 2013, 34-35. - Kozubová 2018, 31-32. - Kozubová 2019, 107-109, 123, 156-157 and Fig. 31. - At the sites of the Vekerzug group, the Ciumbrud-type temple rings are the oldest.

120 MARIĆ 1964, 33, 36 and Pl. VI/16. - The temple ring is considered to be a local form. 


\begin{tabular}{|c|c|c|c|c|c|c|c|c|}
\hline Grave & Ritual & Age & No. & $\begin{array}{l}\text { Diam. } \\
\text { in } \mathrm{cm}\end{array}$ & $\begin{array}{l}\text { Other Types } \\
\text { of Hair-Rings } \\
\text { in Grave and } \\
\text { their Number }\end{array}$ & $\begin{array}{l}\text { Other Important } \\
\text { Costume Items }\end{array}$ & $\begin{array}{l}\text { Other Important } \\
\text { Jewellery Items }\end{array}$ & References \\
\hline N. Čegrlja 3 & INH & $18-25$ & $1+1$ ? & 8.0 & $\mathrm{~A} 1 / / 3+3$ & $\begin{array}{l}\text { plate fibula (2), } \\
\text { spectacle fibula, } \\
\text { double-looped bow } \\
\text { fibula with the foot } \\
\text { in the shape of the } \\
\text { Boeotian shield, open- } \\
\text { work belt }\end{array}$ & $\begin{array}{l}\text { diadem, torc, } \\
\text { amber beads, } \\
\text { bracelet with } \\
\text { stamp-shaped } \\
\text { terminals (2) }\end{array}$ & $\begin{array}{l}\text { TruhelKa 1904, } \\
85-86 \text { and Pl. XL/2. } \\
\text { - MARIĆ 1964, } 40 \\
\text { and Pl. XXV/12. - } \\
\text { Čović 1987a, 241 } \\
\text { and Pl. XXV/1-2. - } \\
\text { GAVRANOVIĆ 2011b, } \\
133 \text { and Fig. 166/2-3. }\end{array}$ \\
\hline M. Petrović Jr. 3 & INH & adult & $1+1$ & $\begin{array}{l}6.5 \\
7.1\end{array}$ & $\begin{array}{l}\mathrm{A} 1 / / 1+1 ? \\
\mathrm{C} 1 / / 1+1 \\
\text { indeterminate } \\
/ / 3\end{array}$ & $\begin{array}{l}\text { boat-shaped fibula, } \\
\text { fibula with amber } \\
\text { pledget (2), plate } \\
\text { fibula, belt set with } \\
\text { buckle and buttons }\end{array}$ & $\begin{array}{l}\text { torc, bracelet with } \\
\text { stamp-shaped } \\
\text { terminals }(2)\end{array}$ & $\begin{array}{l}\text { Truhelka 1904, 90-91 } \\
\text { and Pl. XLII/12-13. } \\
\text { - Marić 1964, 35 } \\
\text { and Pl. XXV/4. - } \\
\text { GAVRAnOviĆ 2011b, } \\
136 \text { and Fig. } 172 / 2-3 .\end{array}$ \\
\hline M. Petrović Jr. 6 & INH & adult & 5 & $\begin{array}{l}6.3- \\
8.0\end{array}$ & $\begin{array}{l}\mathrm{A} 1 / / 1+1 \\
\mathrm{C} 1 / / 4 \\
\mathrm{D} 2 / / 1\end{array}$ & $\begin{array}{l}\text { fibula with the foot } \\
\text { in the shape of the } \\
\text { Boeotian shield (2), } \\
\text { Borajna-type fibula, } \\
\text { fibula with perforation } \\
\text { on the top of the bow } \\
\text { (2), belt set with buckle } \\
\text { and buttons, silver pin }\end{array}$ & $\begin{array}{l}\text { torc, amber beads, } \\
\text { spiral bracelet ( } 2)\end{array}$ & $\begin{array}{l}\text { TruheLKa 1904, 91-92 } \\
\text { and Pl. XLIII/10-11. } \\
\text { - Marić 1964, } 40 \text { and } \\
\text { Pl. XXV/6. }\end{array}$ \\
\hline S. Jakarić 14 & CREM & adult & 5 & $\begin{array}{l}7.2- \\
7.8\end{array}$ & $\mathrm{C} 1 / / 7$ & & $\begin{array}{l}\text { torc }(2) \text {, bracelet } \\
\text { with stamp- } \\
\text { shaped terminals } \\
\text { (2) }\end{array}$ & $\begin{array}{l}\text { TRUhELKa 1904, } \\
\text { 107-108 and Pls. LV/3; } \\
\text { LVI/6. - MARIĆ 1964, } \\
40 \text { and Pl. XXV/10. }\end{array}$ \\
\hline M. Petrović 1 & INH & adult & $4+4$ & 7.5 & & $\begin{array}{l}\text { double-looped fibula } \\
\text { with triangular foot, } \\
\text { Marvinci-Gogoşu- } \\
\text { type fibula, double- } \\
\text { looped fibula with } \\
\text { triangular foot(?), } \\
\text { Donja Dolina-type pin }\end{array}$ & amber beads & $\begin{array}{l}\text { Truhelka 1904, 117- } \\
\text { 118. - Marić 1964, } 40 \\
\text { and Pl. XXV/8. }\end{array}$ \\
\hline M. Petrović 8 & $\mathrm{INH}$ & adult & $3+3$ & 8.5 & $\mathrm{~A} 1 / / 1+1$ & boat-shaped fibula (4) & torc & $\begin{array}{l}\text { Truhelka 1904, 120- } \\
\text { 121. - Marić 1964, } 40 \\
\text { and Pl. XXV/14. }\end{array}$ \\
\hline
\end{tabular}

Tab. 3. Graves from the Donja Dolina cemetery containing temple rings of type B - the Donja Dolina type - with important items of costume and jewellery (M. Dizdar).

temple rings on each side of the head. On the other hand, when there is a single type $\mathrm{B}$ temple ring on each side of the head, it is combined with type A1 and/or C1. Three on each side have been found in combination with type A1 or type $\mathrm{C} 1$; three of them seem to be combined with a single type B temple ring on each side in grave N. Čegrlja 3 (Fig. 2). ${ }^{121}$ The grave M. Petrović Jr. 6 contained five type B temple rings combined with types A1, C1, and D2 (Fig. 4).

121 Čović 1987a, 241 and Pl. XXV/1-2.
As shown by these graves, they almost always have the same number on each side of the head. They measure $6.3 \mathrm{~cm}$ to $8.5 \mathrm{~cm}$ in diameter.

In the Donja Dolina cemetery type B bronze temple rings with decorated terminals have been found in graves of women, often richly furnished with items of costume and ring jewellery (Tab. 3). The majority of these graves belong to phase $2 \mathrm{a}$ after the classification of Čović, or phases 1 and 2 after the classification of Teržan (Figs. 2-3, 8). The younger group of graves with type $\mathrm{B}$ temple rings dated to phase $2 \mathrm{~b}$ 


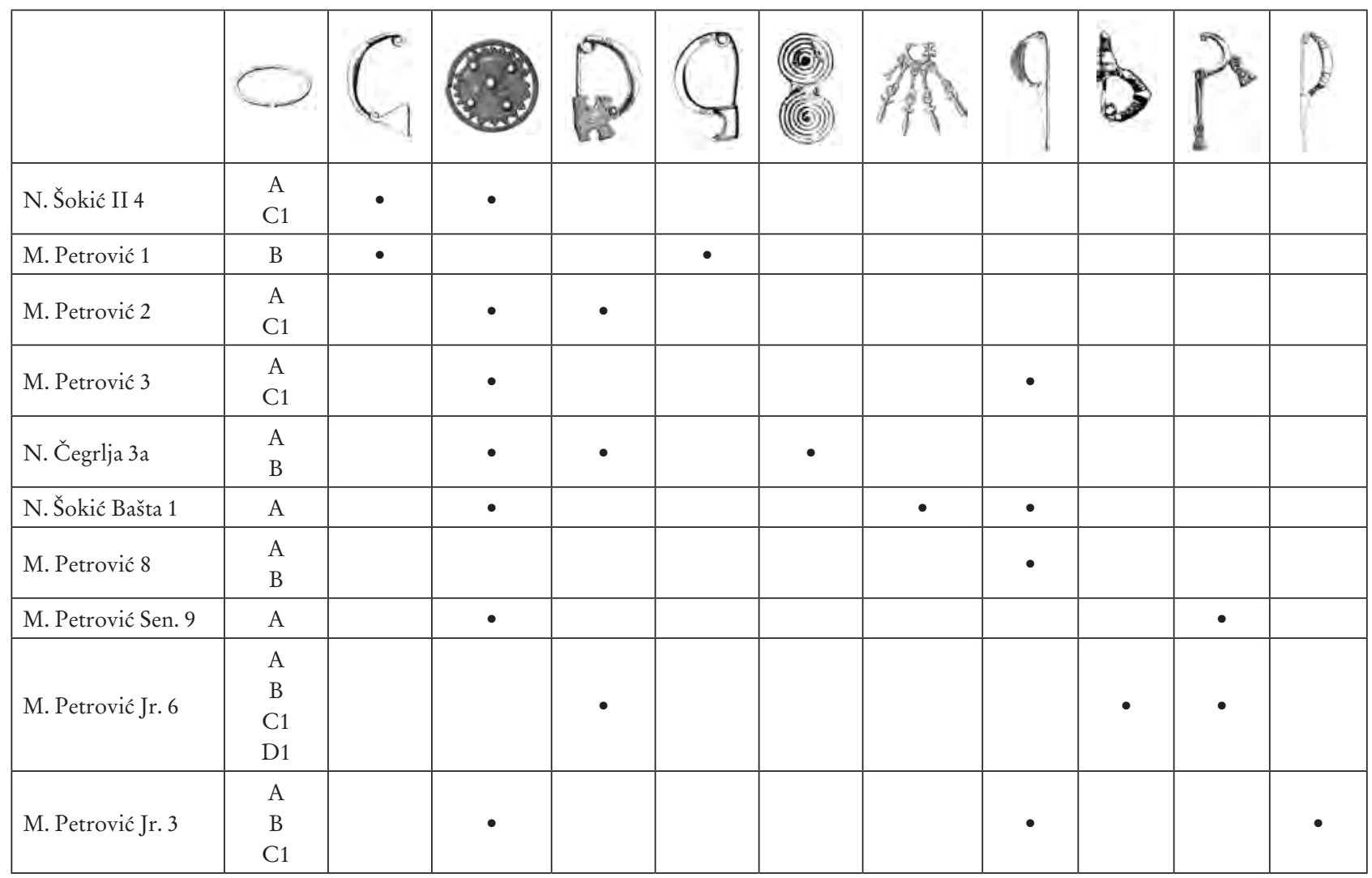

Tab. 4. Types of fibulae that appear in the graves in Donja Dolina with the temple rings of types A and B (A. Kapuran).

after Čović or phase 3 after Teržan (grave M. Petrović Jr. 6). ${ }^{122}$ This will also be shown by the typo-chronological analyses of items of costume and ring jewellery from these graves, which will include the grave assemblages with type A1 temple rings (Tabs. 1, 3).

\section{Costume and Jewellery Items in Graves with Type A and B Temple Rings}

For the chronological and sociological definitions of graves with temple rings of types $\mathrm{A}$ and $\mathrm{B}$, but also other recognized types, it is extremely important to analyse the grave assemblages where such forms appear, primarily in the Donja Dolina cemetery. ${ }^{123}$ Also, the analysis will include other costume and jewellery items from the graves of the Ciumbrud group containing type A temple rings; as already pointed out, at the cemeteries in Transylvania and northeast Hungary they have been dated to the second half of the $7^{\text {th }}$

122 TeržAn 1974, 43-45 and Fig. 7. - Čović 1987a, 238-251. 123 As already stated, we do not analyse other types of hair decorations here, although some of them (types C1, D1a, D1b and D2) occur within the same graves in which were found temple rings of types $\mathrm{A}$ and $\mathrm{B}$, which already indicates they were contemporary. However, these are forms that occur in some younger graves in Donja Dolina, so they will be the subject of a separate analysis. and the first half of the $6^{\text {th }}$ century BC, which matches the chronological insights of Čović, Teržan and Gavranović for the Donja Dolina cemetery.

As temple rings of types A and B in the Donja Dolina cemetery sometimes come from the same graves (Tabs. 1, 3), this will significantly facilitate the analysis of other items of costume and jewellery, with the common appearance of both types indicating that they were worn in the same time frame. Costume items are associated with different forms of belts or belt sets and fibulae, occasionally also with pins. On the other hand, ring jewellery includes the finds of torcs and bracelets (Tabs. 4-5). Other items, especially jewellery, like the different forms of beads or pendants, have not been analysed separately here, but we mention some of the more important forms.

Among the belts in the graves of Donja Dolina which contain temple rings of types $\mathrm{A}$ and $\mathrm{B}$, there are (Tab. 5) open-work belts and belt sets with a buckle and buttons. Among the fibulae (Tab. 4), there are a large number of different forms with greater or smaller numbers of types. Among the ring jewellery, those important for the analyses are torcs, bracelets with stamp-shaped terminals, and spiral bracelets (Tab. 5). 


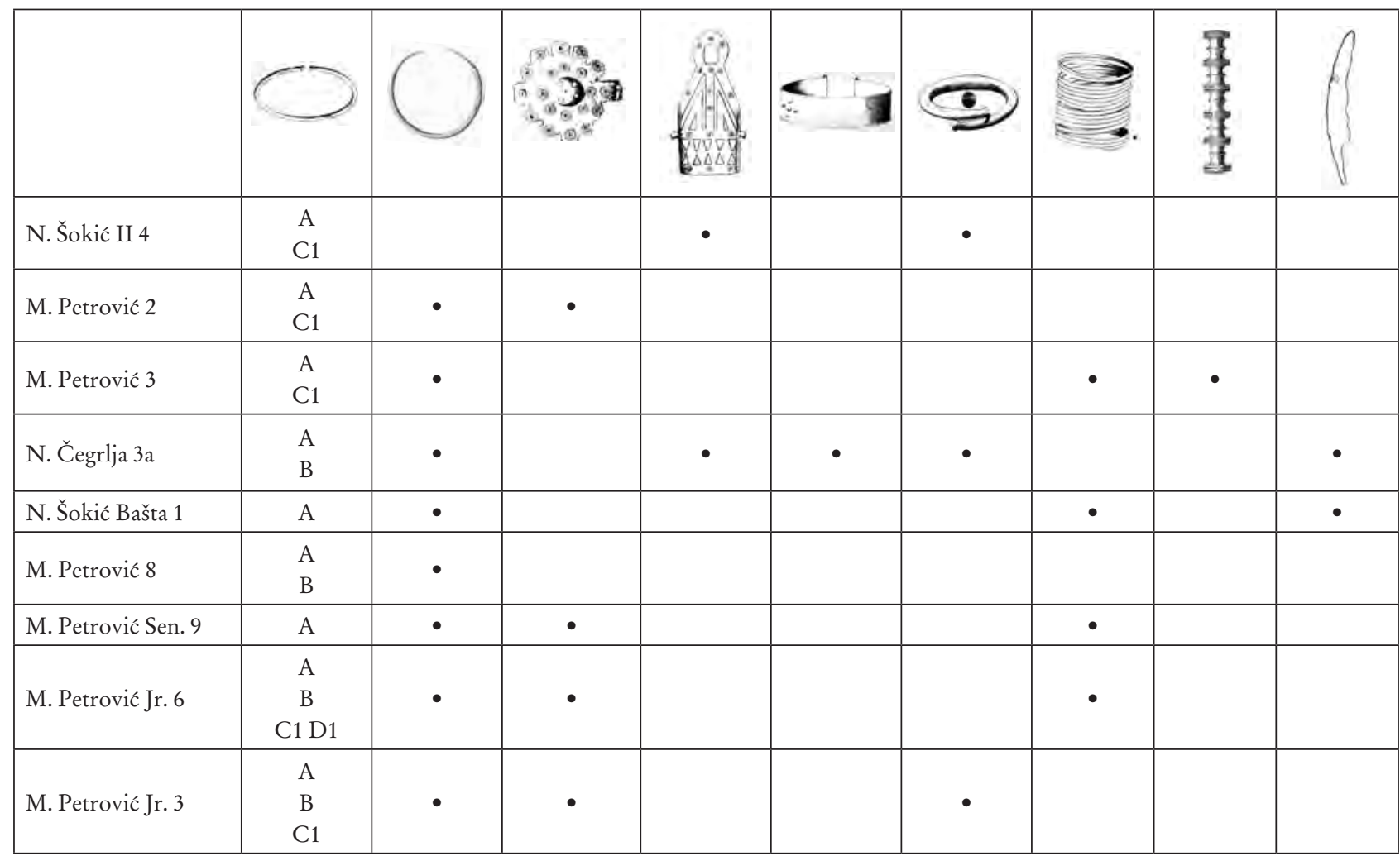

Tab. 5. Costume and jewellery items that appear in the graves in Donja Dolina with the temple rings of types A and B (A. Kapuran).

\subsection{Open-Work Belts}

Open-work belts are known from the two grave assemblages in the Donja Dolina cemetery (Tab. 5) which contain temple rings of types A and B - grave N. Čegrlja 3 (Fig. 2/13-14) 124 and grave N. Šokić II 4 (Fig. 9/7, 10). ${ }^{125}$ Since both of them are inhumation graves, the excavations established that the belts were located in the area of the waist and consisted of buckles and open-work fittings, but also spiral tubes. Another fitting with spiral tubes comes from cremation grave N. Šokić I $8,{ }^{126}$ while one buckle comes from outside the grave assemblage. ${ }^{127}$

These graves with open-work belts, which include a buckle and several rectangular fittings with spiral tubes connecting them, ${ }^{128}$ have been dated after Teržan to the oldest burial horizon in Donja Dolina, which is associated with phase $\mathrm{HaC} 1$, but they continue into the next one. ${ }^{129}$ Čović

124 Truhelka 1904, 85-86 and Pl. XL/13-14.

125 Truhelka 1904, 133 and Pl. LXXVII/13-14, 18.

126 Truhelka 1904, 113 and Pl. LX/37.

127 Marić 1964, 37, 40 and Pl. X/10.

128 The last analysis of open-work belts was presented in: DizDAR 2020.

129 TERŽAN 1974, 43 and Fig. 7. dates the start of the burials at the Donja Dolina cemetery to phase $\mathrm{Ha}$ C2, when open-work belts appear in graves during phase $2 \mathrm{a}$ and continue during the phase $2 \mathrm{~b} .{ }^{130}$ Vasić has several papers on bronze open-work belts, dating them to horizon 2 of the Early Iron Age in Serbia ( $\mathrm{Ha}$ C2), when they are the most numerous and often combined with double-loop fibulae with the foot in the shape of a Boeotian shield or with a triangular foot. Still, belts have also been found in the graves of phase 3, as testified by grave 1 from the Karagač cemetery. Belts have been found at sites from southern Hungary all the way to the Peloponnese and from the Black Sea to Donja Dolina; it is assumed that they originated in Greece. ${ }^{131}$ It is particularly interesting that they have also been found together with bronze bracelets with stamp-shaped terminals, as in the case of the inhumation grave from Hrtkovci, which also included spiral bracelets. ${ }^{132}$

130 Čović 1987a, 241, 246 and Fig. 14/14.

131 Vasić 1971, 1, 4, 6, 8 and Map 1. - Vasić 1977, 17-20, 25, 33. VASIĆ 1987c, 661, 664-664 and Fig. 37/13, Pl. LXVIII/3-4. - Vasić considers them representatives of the 'Zlot decorative style'. - FILIPović 2015, 50 and Map 2. - Dizdar 2020, 193-198.

132 VinSKi 1955, 35 and Fig. 18. - VINSKI, VINSKI-GASPARINI 1962, 271, 278 and Pl. VI/70-71. - Dizdar 2020, 193 and Fig. 3. 
The finds that are the most similar to Donja Dolina are those from the destroyed graves at Dvorovi near Bijeljina, which belong to the younger group of finds. ${ }^{133}$ Finds of belts from northwestern Bulgaria are analysed by Diana Gergova, who singles out a distinctive triangle-shaped open-work decoration with alternating motifs of concentric circles. Three basic variants are distinguished on the basis of buckle shapes. The belts are dated to the second half of the $7^{\text {th }}$ and the first half of the $6^{\text {th }}$ century BC. ${ }^{134}$ A similar opinion and distribution were provided earlier by Klaus Kilian, ${ }^{135}$ followed by Hermann Parzinger. ${ }^{136}$ Analysing the open-work belts from Donja Dolina, Gavranović also points out that they were most widespread between the rivers Morava, Vardar, and Isker; for the finds from Donja Dolina, he recognizes the importance of the communication route along the Sava and the Danube, confirmed by finds from Hrtkovci and Dvorovi. The finds from Donja Dolina have been dated to the $7^{\text {th }}$ century BC; it is stated that they are often combined with double-loop fibulae with the foot in the shape of a Boeotian shield and bronze bracelets with stamp-shaped terminals over a wide territory (Fig. 2), pointing to the existence of super-regional female costume and jewellery. ${ }^{137}$ Interestingly, there are still no belts from the Transylvanian sites, even though a communication route from the confluence of the Sava and the Danube to the north is indicated by the find of an open-work belt with a double-loop fibula with the foot in the shape of a Boeotian shield in the cremation grave at the site of Nyiregyháza-Közvágóhíd. ${ }^{138}$ Moreover, there are no open-work belts from the distribution area of the Glasinac group, even though there are many direct contacts of that area with Donja Dolina, as shown by some contemporary finds such as belt sets consisting of different buckles and buttons (Figs. 3/10-11, 4/14, 6/10, 7/15-18). Bronze open-work belts show direct contacts of Donja Dolina with eastern areas, primarily with the areas of eastern Serbia and, further away, southwestern Romania and northwestern Bulgaria (Fig. 15/1). They are a characteristic female

133 Marić 1960, 50-54 and Pls. II/3; III/1-5. - Gavranović 2011a, 220. - Dizdar 2020, 196 and Fig. 7. - With fibulae of the Ararava gromila type.

134 Gergova 1987, 61-63 and Pls. 22-24/A30-A40. - BADER 1983, 85 , mentions the finds from the Ferigile cemetery (Pl. 60A-B). HRIsTOV 2004 with new finds of belts (Fig. 15/1).

135 Kilian 1975, 61, 89, 95-96, 108 and Pl. 53/3-9; showing the distribution by that time at Pl. 84/1.

136 Parzinger 1992, 234 and Fig. 10/2.

137 Gavranović 2011a, 219-220 and Fig. 220.

138 Kemenczei 2009, 86, 130 and Pl. 49/4, pointing out the comparisons with the finds from the northern Balkans, while the grave has been dated to the second half of the $7^{\text {th }}$ and the first half of the $6^{\text {th }}$ century BC. costume item in Donja Dolina, found in the oldest graves, which can be dated to the second half of the $7^{\text {th }}$ century BC.

\subsection{Belt Sets with a Buckle and Buttons}

Belt sets consisting of different forms of buckles and buttons are known from several graves in Donja Dolina that contained temple rings of types A and B (Tab. 5), often together with those of type C1. Grave M. Petrović Jr. 3 contained a round conical belt buckle, $6.5 \mathrm{~cm}$ in diameter, with a hemispherical protruding central part, under which there is a hole on the bottom side, while the front side is decorated with incised circular lines (Fig. 3/10). The belt also includes calotte buttons with holes, organized in four rows (Fig. 3/11). ${ }^{139}$ Grave M. Petrović Jr. 6 (Fig. 4/14), ${ }^{140}$ grave M. Petrović 2 (Fig. 5/3, 11), ${ }^{141}$ and grave M. Petrović Sen. 4 (Fig. 6/10) ${ }^{142}$ contained round belt buckles with a hemispherical protruding central part surrounded by a decoration of concentric circles; along the edge there are petal-shaped protrusions decorated with the motif of concentric circles or a ring-and-dot motif. The buckle of this kind, with a central strip which is curved on the edges and which fastened the buckle to the belt, decorated with a cross motif on the upper side, was found in the richly furnished cremation grave N. Šokić I $1 .{ }^{143}$ There is also another buckle from Donja Dolina. ${ }^{144}$

The belt buckle from grave M. Petrović Jr. 3 is associated with buckles of type 1, dated from phase Glasinac IVa to phase IVc-1. They are buckles that are based on the richly decorated conical buckles of the late $9^{\text {th }}$ and the $8^{\text {th }}$ century BC. ${ }^{145}$ The buckles from the remaining three graves are associated with type 6 , characterized by petals ordered along the edge, while the hemispherical central part is protruding. Buckles of type 6 are dated to the end of the $7^{\text {th }}$ and the $6^{\text {th }}$ century BC; they are often found in female graves, ${ }^{146}$ as also testified to by the finds from the Donja Dolina cemetery.

Teržan dated the graves with belt sets to the horizon following the graves she believes to be characteristic for phase $\mathrm{Ha}$ C2, meaning that she believes they are younger than the open-work belts; they are accompanied by Borajna-type fibulae or fibulae with a ring at the top of the bow,

139 Truhelka 1904, 90 and Pl. XLII/23-24.

140 Truhelka 1904, 91 and Pl. XLIV/1-2.

141 Truhelka 1904, 118. - Gavranović 2011b, 128 and Fig. 158/3,

13. - Probably combined with calotte buttons with slits.

142 Truhelka 1904, 122-123 and Pl. LXVIII/22-23.

143 Truhelka 1904, 111 and Pl. LIX/1-2.

144 Marić 1964, Pl. VI/14. - Gavranović 2011a, 224 and Fig. 225/1.

145 Tessman 2004, 142, 146 and Fig. 3/5.

146 Tessman 2004, 144 and Fig. 13. 
which she dates to Ha D1. ${ }^{147}$ However, the aforementioned graves indicate that it is a belt type that actually appears at the same time as the open-work belts, only with a different origin, with the buckle from grave M. Petrović Jr. 3 certainly belonging to the older type. This buckle has been dated through amber-coated fibulae and a boat-shaped fibula to the second half of the $7^{\text {th }}$ century BC. ${ }^{148}$ Buckles of type 6 , which Čović also calls the Glasinac type, have also been dated to phases $2 \mathrm{a}$ and $2 \mathrm{~b} .{ }^{149} \mathrm{He}$ reaches a similar conclusion in analysis of the buckles from the Glasinac area and believes them to be a characteristic item from phase Glasinac IVb; however, they also appear in phase IVc-1, when they have a protruding calotte-shaped central part and petals along the edge, while those without petals along the edge appear in smaller numbers. ${ }^{150}$ Round belt buckles with petals are also considered by Gavranović and he dates them to the second half of the $7^{\text {th }}$ and the early $6^{\text {th }}$ century BC, emphasizing their frequent appearances at Glasinac and in western Serbia. ${ }^{151}$ Vasić dates buckles with edge petals to the contemporary phase 2 of the Early Iron Age in Serbia, ${ }^{152}$ while Parzinger points out that belt sets of the Glasinac-Mati type have been found in the area from Glasinac all the way to northern Albania, with Donja Dolina as the farthest northern site. ${ }^{153} \mathrm{It}$ has been noted that they have their greatest concentration at Glasinac, and dated from phase Glasinac IVb to phase IVc2 , meaning to the $7^{\text {th }}$ and $6^{\text {th }}$ centuries BC, continuing until phase Va. ${ }^{154}$

In relation to type 6 belt buckles, a somewhat different buckle has been found in inhumation grave M. Petrović Sen. 9. It is the second belt from that grave which is assumed to have possibly been a gift to the deceased woman. This buckle has edge petals too, while the central part is T-shaped and decorated with open work. Next to the buckle, there were single and double calotte buttons with radial slits

147 TeržAn 1974, 43 and Fig. 7.

148 Gavranović 2011a, 219.

149 Čović 1987a, 241, 243, 246 and Fig. 14/15, Pls. XXIV/17; $\mathrm{XXVI} / 10$. - In phase $2 \mathrm{~b}$, the buckles are accompanied by single or double calotte buttons with radial slits.

150 Čović 1987b, 596, 610 and Fig. 35/7-8, 23-24, Pl. LXI/7-8, 19. Also: Kilian 1975, 108 and Pls. 82/3; 87. - LuCEnTini 1981, 83 and Fig. 4/13.

151 Gavranović 2011a, 223-224 and Fig. 225. - As a part of the set, buckles are accompanied by calotte buttons that can be followed from the older phase of the Urnfield culture to the later phase of the Early Iron Age.

152 VAsić 1977, 18, 21 and Pls. 26/6; 28/4. - E.g. Pilatovići tumulus III, grave 6, the finds from the tumulus in Uzići etc.

153 Parzinger 1992, 234 and Fig. 11/1.

154 Blečić Kavur, MiličEvić-Capek 2011, 46, 50, 52 and Fig. 10.
(Fig. 7/17-18). ${ }^{155}$ This buckle has been dated to phase $2 b^{156}$ and associated with buckles of type 6, variant 3, characterized by an open-work central part, while the petals are decorated with a concentric circle motif. The buckles of this variant have been dated to the $7^{\text {th }}$ and $6^{\text {th }}$ centuries BC. ${ }^{157}$ Therefore, it is a younger form of buckles, which first appeared at Glasinac, ${ }^{158}$ with grave M. Petrović Sen. 9 being dated to the late $7^{\text {th }}$ century BC. ${ }^{159}$ Along with the buckle of the second belt from the grave 9 , there are single and double calotte buttons with radial slits. These buttons were made in several sizes and variants (single, double, or triple), and have been dated to phase IVc-1, continuing into phase IVc2 , when they are less numerous, even appearing singly in graves. ${ }^{160}$ Aside from their function within belt sets, buttons of this kind could have served as armour when sewn onto the clothes. ${ }^{161}$ They are distributed from eastern Bosnia all the way to Greece, ${ }^{162}$ with Donja Dolina still being the farthest northwestern site where they appear in the graves of the late $7^{\text {th }}$ and the $6^{\text {th }}$ century BC. ${ }^{163}$ In grave 9 , the deceased woman wore a belt set consisting of a spherical open-work buckle with a cross motif and a large hole on the bottom side and single and double calotte buttons with radial slits (Fig. 7/1516). ${ }^{164}$ The grave is associated with phase $2 b,{ }^{165}$ or the late $7^{\text {th }}$ century BC. ${ }^{166}$ Interestingly, this grave also contained three buckles in the shape of a rosette with a calotte-shaped central part and a crossbar on the bottom side. There are spiral tubes between the buckles (Fig. 7/13). ${ }^{167}$ Rosette-shaped buckles have been found in several other graves in Donja Dolina, often with spiral tubes, ${ }^{168}$ including the interesting find of a buckle in inhumation grave N. Šokić II 4, which has two fittings belonging to an open-work belt (Fig. 9/8). ${ }^{169}$ These buckles are considered to be a new form which

\footnotetext{
155 Truhelka 1904, 124 and Pl. LXXII/1-2.

156 Čović 1987a, 246 and Pl. XXVI/7.

157 Tessman 2004, 145, 152 and Fig. 16.

158 Lucentini 1981, 83 and Fig. 4/10. - Like type A, variant 1.

159 Gavranović 2011a, 223 and Fig. 224/1.

160 Čović 1987b, 596, 610, 620 and Fig. 35/10-11, Pl. LXI/2-4.

161 Kilian 1975, 51. - Zotović 1985, 84. - Čović 1987b, 596.

162 Kilian 1975, 28, 109 and Pl. 87/4. - LuCENTINI 1981, 80 and Fig. 3/30-31.

163 Gavranović 2011a, 232 and Fig. 234/3.

164 Truhelka 1904, 124 and Pl. LXXI/8-9.

165 Čović 1987a, 248.

166 Gavranović 2011a, 239.

167 Truhelka 1904, 124-125 and Pl. LXXI/2, 4-5.

168 Grave N. Šokić I 1: Truhelka 1904, 111 and Pl. LVIII/14, 22, 25-26. - Grave M. Petrović Jr. 20: Truhelka 1904, 127 and Pl. LIII/3. - Grave M. Petrović Jr. 35: Truhelka 1904, 98 and Pl. XLVIII/19. Grave M. Petrović Jr. 59: Truhelka 1904, 105 and Pl. LIII/21-22, 24. 169 Truhelka 1904, 133 and Pl. LXXVIII/20.
} 
appears in phase $2 \mathrm{~b},{ }^{170}$ and they are considered as part of a belt or a chest decoration appearing in the graves of the late $7^{\text {th }}$ and the early $6^{\text {th }}$ century BC. ${ }^{171}$ It is believed they originated from the areas of Greece and Macedonia, where they are particularly numerous in the $7^{\text {th }}$ and $6^{\text {th }}$ centuries $\mathrm{BC} ;{ }^{172}$ there is an interesting find in the Vekerzug group cemetery of Muhi-Kocmadom, with parallels in the northern Balkans and Donja Dolina. ${ }^{173}$

Thus, belt sets consisting of differently shaped buckles and buttons reveal direct contacts with the Glasinac area in the south and are a characteristic female costume item in Donja Dolina, where they have been found in graves that can be dated to the second half of the $7^{\text {th }}$ century and the beginning of the $6^{\text {th }}$ century BC.

\subsection{Double-Loop Fibulae with a Triangular Foot}

As we already pointed out, in dating the Donja Dolina graves with temple rings of types A and B, particular importance is given to the finds of various forms of fibulae (Tab. 4), which should definitely be joined by the other finds from the Ciumbrud group cemeteries. The first to be analysed are bronze double-loop fibulae with a triangular foot, which can be classified into several variants depending on the form and cross section of the bow.

Grave N. Šokić II 4 contained a bronze fibula with a rounded cross section to the bow and a triangular foot. The pin of the fibula is missing and it was fastened with an iron rivet (Fig. 9/5). ${ }^{174}$ Grave M. Petrović 1 contained a fibula with a leaf-like hammered and decorated bow of strap cross section, and probably another fibula with a leaf-like bow and a missing foot. ${ }^{175}$ Fibulae with a triangular foot have also been found in some other graves at the Donja Dolina cemetery. Grave M. Petrović Sen. 10 contained a fibula with a larger triangular foot and ribbed bow, with the ribs in the middle of the bow being a little larger and wider. ${ }^{176}$ We should also mention the fibula with ribs at the terminals of the bow, decorated along the edges with a tremolo line, found in the richly furnished grave N. Šokić I 1, ${ }^{177}$ and dated to phase $2 \mathrm{~b} .{ }^{178}$ Found outside the grave assemblages were

170 Čović 1987a, 246 and Pl. XXVI/9.

171 Gavranović 2011a, 233 and Fig. 236.

172 Kilian 1975, 84, 109 and Pl. 90/2. - Gergova 1987, 60 and Pl. 22/A17-A20.

173 Kemenczei 2009, 86, 128 and Pl. 42/4.

174 Truhelka 1904, 133 and Pl. LXXVII/21.

175 Truhelka 1904, 117. - Marić 1964, Pl. IX/10, 13. - Truhelka

wrote that one fibula had a foot in the shape of a Boeotian shield.

176 Truhelka 1904, 125 and Pl. LXXII/18. - Gavranović 2016, 132 and Fig. 3 dates the grave to the early $7^{\text {th }}$ century BC.

177 Truhelka 1904, 110 and Pl. LVIII/3.

178 Čović 1987a, 246 and Fig. 15/12. a fibula with a twisted bow and a larger triangular foot ${ }^{179}$ and a fibula with a rhombic cross section to the bow with ribbed terminals and a larger triangular foot, for which it is not quite clear if it comes from grave I. Stipančević 17. 180 We should also mention the remains of a double-loop fibula without the preserved foot from the cemetery of Proștea Mică (Tirnăvioara) with the find of a type A temple ring. ${ }^{181}$

Fibulae of this kind in the Donja Dolina cemetery have been placed in the oldest burial horizon, matching phase $\mathrm{Ha} \mathrm{C1;}{ }^{182}$ in Serbia, they have been dated in horizon $2 .{ }^{183} \mathrm{Ti}$ beriu Bader classifies double-loop fibulae with a triangular foot into four variants considering the cross section of the bow, which can be found both in female and male graves. They are usually found singly on the shoulders or chest of the deceased, but they have been found in pairs too. ${ }^{184} \mathrm{Simi}-$ larly, Vasić classifies these fibulae into three variants considering the cross section of the bow. ${ }^{185}$ These fibulae have also been classified into several variants by Gavranović, who dates them to the period from the $8^{\text {th }}$ to the $6^{\text {th }}$ century BC. ${ }^{186}$

The fibula with a triangular foot and twisted bow from Donja Dolina which does not come from a grave belongs to type $7 \mathrm{a}$ after the classification of Stane Gabrovec. ${ }^{187}$ These fibulae are considered to be a somewhat younger form within phase Glasinac IVa. The younger forms of fibulae are characterized by a finely twisted bow, and continue into phase IVc-1. ${ }^{188}$ Fibulae of type 7 a have been found at Danube sites all the way to western Bulgaria, where they have been dated mostly to the $7^{\text {th }}$ century BC, then to Macedonia and Greece in the south, while there are none in the southeast

179 Marić 1964, Pl. VI/9. - Gavranović 2011b, 80 and Fig. 102/1. 180 Marić 1964, Pl. IX/9.

181 BADER 1983, 73 and Pl. 24/142. - KemencZei 2004, 90 and Fig. 4/1.

182 TeržAn 1974, 43 and Fig. 7. - The same opinion: PARZinger 1992, 231 and Fig. 6, dating to the second half of the $8^{\text {th }}$ century BC and Gavranović 2011a, 263, 265. - Gavranović 2016, 130, 132.

183 VASIĆ $1977,18$.

184 BADER 1983, 71, 76-77 and Pl. 47. - Fibulae of this kind have been dated to the $8^{\text {th }}$ century BC in Greece, while they appear in Bulgaria in the second half of the $7^{\text {th }}$ century BC. At the sites in Romania, older forms appear at the cemeteries of Basarabi and Balta Verde, while younger ones appear at the Gogoşu cemetery. They are distributed at sites across southeastern Europe (Pl. 47), and in particular in the Balkans and Romania.

185 VAsIĆ 1999, 49. - Smooth-bowed fibulae may have a round or square-shaped bow that is sometimes decorated with transverse lines. 186 Gavranović 2011a, 184 and Fig. 174.

187 Gabrovec 1970, 29, 37-38 and Map X.

188 Čović 1987b, phase IVa: 585-586, 595, 607 and Fig. 33/9; 34/27, Pl. LX/9. 
Alps. ${ }^{189}$ A fibula from Dalj has a smaller triangular foot. ${ }^{190}$ Double-loop bow fibulae from the Bulgarian sites are classified into several variants considering the decoration and cross section of the bow; the foot is mostly undecorated, but it can include hammered decorations, concentric circle motifs, or zig-zag lines. The fibulae of type B I 2, variant $\beta$, are characterized by a twisted bow and a larger or smaller triangular foot that can be decorated with a concentric circle motif. These fibulae are distributed across the central and eastern Balkans, where they have been dated to the $7^{\text {th }}$ century BC. ${ }^{191}$ Fibulae with a twisted bow belong to variant D, which is known from sites in Bulgaria, Greece and Serbia, all the way to Donja Dolina. ${ }^{192}$ These fibulae, according to the classification of Vasić, encompass a heterogeneous group of finds, with the nearest parallels to the one from Donja Dolina are the fibulae from Pilatovići and Šabac, which are smaller. Fibulae of this type are distributed in eastern Bosnia, Serbia, and western Bulgaria, being most common during the $7^{\text {th }}$ century BC. ${ }^{193}$ A fibula with a twisted bow has been found in the Hallstatt cemetery too; its triangular foot is decorated with a tremolo decoration. ${ }^{194}$

For fibulae with a rhombic cross section to the bow and a triangular foot, singled out as type $3 a,{ }^{195}$ a parallel has been identified in Dalj; a fibula has a smaller triangular foot, and the spiral towards the pin is connected with the bow by means of two rivets. ${ }^{196}$ Bader distinguishes fibulae with a square cross section to the bow as variant $B$, while the fibula from Donja Dolina (grave I. Stipančević 17?) with ribs on the ends of the bow could be close to a fibula from Basarabi/Gogoșu. It is pointed out for the fibulae of this variant that they are found at sites in Bulgaria and southwestern Romania. ${ }^{197}$ Gergova distinguishes double-loop fibulae of type B I 2, variant $\gamma$, which have a rhombic cross section to the bow and a foot that is triangular but can occasionally be square. The bow is slightly expanded in the middle and always undecorated, with a geometric decoration found only once. The fibulae of this kind are distributed in the eastern Balkans and dated from the beginning of the $7^{\text {th }}$ to the middle of the $6^{\text {th }}$ century BC. ${ }^{198}$ On the other hand,

189 GaVRaNOVIĆ 2011a, 185.

190 VinsKi, VinSKi-GASPARINI 1962, 270, 275 and Pl. VII/75.

191 Gergova 1987, 36, 39-40 and Pl. 9/114-121.

192 BADER 1983, 75, 77 and Pl. 25/154.

193 VAsić 1999, 51-52 and Pl. 27/323-325, Map 63B.

194 GLunz 1997, 47, 168 and Pl. 8/2. - The fibula from Donja Dolina is said to be single-looped.

195 Gabrovec 1970, 24 and Map VI.

196 VINSKI, VINSKI-GASPARINI 1962, 270, 275 and Pl. VII/76

197 BADER 1983, 74-75, 77 and Pl. 25/152.

198 Gergova 1987, 40-41 and Pls. 9/122-126; 10/127-134A. bronze fibulae with a rhombic cross section to the bow and a triangular foot have been dated from the first half of the $8^{\text {th }}$ to the middle of the $7^{\text {th }}$ century BC at the latest. ${ }^{199}$

Fibulae with a rounded cross section to the bow and a triangular foot are distinguished as type 1c. ${ }^{200}$ The entire bow or only its ends are often decorated with tiny transversal ribs, like the example from Donja Dolina. The fibula from grave N. Šokić I 1 has a parallel in the fibulae from Pilatovići, which have a larger triangular foot, and the foot of one fibula is also decorated along the edges with a tremolo line. These fibulae are sometimes found in pairs; more often, however, they are single on the chest of the deceased woman. The fibulae have been dated to the second half of the $8^{\text {th }}$ and the first half of the $7^{\text {th }}$ century BC, but they continue into the $6^{\text {th }}$ century BC. It is pointed out that they are distributed in the central Balkans and the southeastern Alpine area, ${ }^{201}$ but here we can also distinguish some older forms that have nothing to do with the fibulae of this type. After the classification of Bader, fibulae with a smooth rounded cross section to the bow belong to variant $\mathrm{A}$. The fibula with a smooth bow from grave N. Šokić II 4 (Fig. 9/5) has a parallel in the one from the surroundings of Aiud, while the fibula with the ribbed ends to the bow from grave N. Šokić I 1 has parallels in the fibulae from the graves in Gogoșu, which also still have ribs in the middle of the bow. ${ }^{202}$ On the other hand, the fibula from the grave in Proștea Mică (Tirnăvioara) has a smooth bow, but the foot has not been preserved, and it has been dated to the middle of the $6^{\text {th }}$ century BC. ${ }^{203}$ Gergova distinguishes double-loop fibulae with a rounded cross section to the bow as variant B I $2 \delta$, which is usually undecorated, while one fibula has a group of transverse grooves on the ends of the bow. Sometimes there are groups of transverse lines on the foot. Fibulae of this kind are distributed in the Balkans and dated to the $7^{\text {th }}$ and $6^{\text {th }}$ centuries $\mathrm{BC}$, but their first appearance was already recorded at the end of the $8^{\text {th }}$ century $\mathrm{BC}$, while the youngest find has been dated to the beginning of the $5^{\text {th }}$ century BC. ${ }^{204}$ Fibulae such as the one found in grave N. Šokić II 4 are dated by Gavranović to the period from the end of the $8^{\text {th }}$ to the

199 TERŽAN 1990, 73, 75.

200 Gabrovec 1970, 24 and Map IV.

201 VASIć 1999, 49-50 and Pl. 26/299-301. - In grave 5 in tumulus V at the Pilatovići-Ravni lug cemetery four spectacle fibulae without a figure-of-eight loop were found. For their distribution: VAsić 1999, Pl. 63B.

202 BADER 1983, 71-73, 77 and Pls. 23/128; 23/132-133; 24/134, 138. 203 BAder 1983, 73 and Pl. 24/142. - KemenCZei 2004, 90 and Fig. 4/1.

204 Gergova 1987, 41-43 and Pls. 10/135-143; 11/144-153; 12/154163. 
developed $7^{\text {th }}$ century BC, pointing out that it is one of the oldest graves in Donja Dolina. These fibulae are distributed in the eastern and central Balkans. ${ }^{205}$ On the other hand, the fibula from grave M. Petrović Sen. 10 with a rib-decorated bow has been dated through other finds, such as the 'Macedonian pendants', to the late $8^{\text {th }}$ and the first half of the $7^{\text {th }}$ century BC. In fact, the quoted parallels are the mentioned finds from the cemeteries in Pilatovići and Gogoșu, which, on the other hand, have been dated to the late $7^{\text {th }}$ and the early $6^{\text {th }}$ century BC. The fibula from grave 10 can be joined by the one from grave N. Šokić I 1, which also has rib decorations on the terminals of the bow, while the foot is decorated with a tremolo line along the edges. Because of other finds, this grave is also a representative of the female costume of the late $7^{\text {th }}$ and the early $6^{\text {th }}$ century BC..$^{206}$

The fibulae with a leaf-like strap-section hammered bow and a triangular foot from grave M. Petrović 1 have been dated to phase $2 \mathrm{~b}$ when considered to be an 'archaic form' continuing from a previous phase. ${ }^{207}$ There are parallels in the finds from a destroyed grave from Vrtište ${ }^{208}$ and Zlot cave. ${ }^{209}$ Vasić believes that these fibulae were actually made in the area of eastern Serbia; in them, he recognizes the connection with the fibulae from grave M. Petrović 1, and dates them to the first half of the $6^{\text {th }}$ century BC, just like the mentioned grave from Donja Dolina on the basis of the find of a Marvinci-Gogoșu-type fibula. ${ }^{210}$ On the chest of the deceased woman in the inhumation grave from tumulus 43 in the Gogoșu cemetery, there was a single-loop fibula with a leaf-like bow decorated with a tremolo line along the edges and an irregularly drawn zig-zag line through the middle. The fibula is small $(3.8 \mathrm{~cm})$ and has been dated to the first half of the $6^{\text {th }}$ century BC, partly on the basis of the fibulae from grave M. Petrović 1. Fibulae of this kind have been found, after Bader, at the sites in southern Romania, northern Serbia, and Donja Dolina. ${ }^{211}$

205 Gavranović 2011a, 186 and Fig. 174/7.

206 Gavranović 2011a, 186, 188 and Fig. 174/8-9. - Gavranović 2016, 132 and Fig. 3.

207 Čović 1987a, 246.

208 VASIĆ 1977, 18-19 and Pl. 21/5. - VASIĆ 1987c, 661 and Pl. LXVIII/15. - VASIĆ 1999, 53 and Pl. 27/333. - The bow is rhombic in cross section and decorated with a tremolo line in the middle and a zig-zag line along both edges. The grave probably also contained a spectacle fibula with no figure-of-eight loop and a fibula with the foot in the shape of a Boeotian shield.

209 VAsić 1977, 18-19 and Pl. 20/7. - VAsić 1999, 53 and Pl. 27/334. The fibula is decorated with a tremolo line along the edge of the bow and along the edges of the foot.

210 VAsić 1999, 53-54 and Pl. 63B.

211 Vulpe 1967, 197 and Pl. XXIII/3-4. - BADER 1983, 103-104 and

Pl. 34/304. - Vulpe 1977, Fig. 18A/a.
Thus, bronze double-loop bow fibulae, with various shapes of the bow and the triangular foot, point to direct contacts with eastern areas, primarily the area of Serbia and, further away, southwestern Romania and northwestern Bulgaria. They are a characteristic item of the female costume in Donja Dolina and have been found in the oldest graves that have been dated to the middle and the second half of the $7^{\text {th }}$ century BC.

\subsection{Double-Loop Fibulae with the Foot in the Shape of a Boeotian Shield}

In dating graves with temple rings of types $A$ and $B$, particular importance is given to the finds of bronze double-loop fibulae with the foot in the shape of a Boeotian shield, which come singly or in pairs (Tab. 4). A part from the fibulae from the Donja Dolina cemetery, finds from Dobra (Fig. 11/4) and from the cemeteries of the Ciumbrud group (Fig. 12/1) are also known. Considering the form and cross section of the bow and the form of the foot, these fibulae can be classified into several variants. ${ }^{212}$

On the right side of the chest of the deceased woman in grave N. Čegrlja 3 there was a fibula with a rounded cross section to the bow that is ribbed on the terminals on both sides. The foot is decorated along the middle with a rib flanked on each side by a tremolo line also found along the plate edges (Fig. 2/6). ${ }^{213}$ On the chest of the woman in grave M. Petrović Jr. 6 there were two fibulae with a rounded cross section to the bow that are decorated with hatched triangles in the middle part, and there are groups of thickly set lines on each side. Each terminal of the bow has a single ring-like expansion. One fibula has a preserved part of the foot with a $\mathrm{V}$ rib along the middle and two grooves on each side of it (Fig. 4/3-4). ${ }^{214}$ Grave M. Petrović 2 contained two fibulae. One fibula has ring-like ribs on the terminals of the bow, while the other fibula has tiny ribs only before the loop that precedes the foot. The feet are decorated with a $\mathrm{V}$ motif made of shallow grooves (Fig. 5/7, 9). ${ }^{215}$ Fibulae of this kind have also been found in some graves in Donja Dolina: two fibulae with the $\mathrm{V}$-motif foot decoration from the cremation

212 Heilmann 2016 has a detailed classification of these fibulae, recognizing three basic types. She places their appearance in the first half of the $7^{\text {th }}$ century BC, with origins in northwestern Bulgaria and southwestern Romania.

213 Truhelka 1904, 85 and Pl. XL/6. - Gavranović 2016, 132 and Fig. 4 who dates the grave to the second half of the $7^{\text {th }}$ century BC. 214 Truhelka 1904, 91 and Pl. XLIII/7, 9.

215 Truhelka 1904, 118. - Gavranović 2011b, 128 and Fig. 158/5, 7. - There may be another fibula of this type in the grave, but only the bow is preserved. 
grave N. Šokić I 8, ${ }^{216}$ while the destroyed grave 31 contained a fibula similar to the one from grave N. Čegrlja $3 .{ }^{217} \mathrm{Also}$, it is stated that the possible grave I. Stipančević 17 contained a fibula that seems to have an octagonal cross section to the bow. ${ }^{218}$

Type A temple rings with fibulae of this type have also been found in some other graves. In a possible grave from Dobra (Kožica I) (Fig. 11/4) was a fragment of a fibula with a hexagonal cross section to the bow with decorated terminals. ${ }^{219}$ Inhumation grave IV at the Cipău-Gară cemetery contained a fibula foot decorated with a $\mathrm{V}$ motif (Fig. 12/1), ${ }^{220}$ while the inhumation grave from Târgu Mureș contained a fibula with the foot and the pin spiral fastened to a round-section bow with a single rivet each, while the foot is decorated with vertical ribs. ${ }^{221}$

Double-loop fibulae with the foot in the shape of a Boeotian shield have been singled out as group 8, and are assumed to have been created at Glasinac. ${ }^{222}$ In the Donja Dolina cemetery, they have been associated with the Ha C2 phase, ${ }^{223}$ while Čović dates them to phases $2 \mathrm{a}$ and $2 \mathrm{~b} .{ }^{224}$ Vasić dates them to phase 2; they have a large distribution, being most numerous from western Bulgaria to Glasinac. ${ }^{225}$ In his last analysis, Vasić does not distinguish variants, even though he notes the existence of certain differences, like numerous variations in the design of the bow and the foot. When found in graves, they are single or in pairs, with sometimes even four to six fibulae of this type. He dates the oldest finds to the middle of the $7^{\text {th }}$ century BC, but this is actually a characteristic form of fibula for the second half of the $7^{\text {th }}$ and the first half of the $6^{\text {th }}$ century BC, while the youngest finds date from the beginning of the $5^{\text {th }}$ century BC. It is noticeable that the older ones are somewhat larger, while the younger ones are smaller. They are most numerous from eastern Bosnia to northwestern Bulgaria, reaching

216 Truhelka 1904, 113 and Pl. LX/38.

217 Truhelka 1904, 116 and Pl. LXV/25.

218 Marić 1964, Pl. VI/10. - In a possible grave with a double-loop fibula with a triangular foot (Marić 1964, Pl. IX/9).

219 Popović 1984, 135 and Fig. 1. - VAsić 1999, 66 and Pl. 34/446.

220 Vlassa 1961, 28 and Fig. 6/1. - BADER 1983, 87 and Pl. 31/239. -

Kemenczei 2004, 79, 81 and Fig. 2/6. - Heilmann 2016, 16 and Fig. 4 - as variant 2.

221 Bader 1983, 85 and Pl. 29/229. - Kemenczei 2004, 79 and Fig. 2/3.

222 Gabrovec 1970, 32, 39, 42 and Map XI.

223 Teržan 1974, 43 and Fig. 7.

224 Čović 1987a, 241, 243, 246 and Fig. 15/9, Pl. XXV/5-6. Gavranović 2011a, 190-191 and Fig. 174/12-17. - Heilmann 2016, 20.

225 Kilian 1975, 108 and Pl. 83/1. - Vasić 1977, 17, 20, 33. the Dolenjska region in the west and northern Albania and Greece in the south. ${ }^{226}$ At Glasinac, these fibulae are the most numerous and the leading form of phase IV $\mathrm{c}-1$, and it is believed they were created at the beginning of the second half of the $7^{\text {th }}$ century BC. They appear more frequently in graves without weapons, but there are some in graves with weapons too. It is one of the leading types of fibulae of the late $7^{\text {th }}$ and the first half of the $6^{\text {th }}$ century BC, which rarely continues into phase IVc-2, when they disappear. ${ }^{227}$

Bader classifies these fibulae according to the bow cross section, which can be round or octagonal. The greatest number of finds from the Romanian sites is known from the graves of both sexes, with the remark that sometimes they come in pairs in female graves. The fibulae have been dated to the second half of the $7^{\text {th }}$ and the first half of the $6^{\text {th }}$ century BC, with a noticeably smaller number of finds in the area of Transylvania as opposed to more southerly sites. ${ }^{228}$ Gergova distinguishes these fibulae as type B II 2, which is classified into five variants according to the cross section and decoration of the bow and the foot. ${ }^{229}$ Some fibulae of variant $\gamma$ are particularly interesting because they have a wider and lower foot, decorated with the $\mathrm{V}$ motif consisting of lines or ribs, while the round-section middle part of the bow is decorated with the motif of hatched triangles, and the sides have thick and low transverse ribs. In fact, a pair of fibulae decorated in this way has been found in grave M. Petrović Jr. 6 (Fig. 4/3-4). Fibulae of variant $\gamma$ are known from the sites in northwestern Bulgaria, in Macedonia, at Glasinac, and in Donja Dolina, all the way to Hungary, and they have been dated to the second half of the $7^{\text {th }}$ and the beginning of the $6^{\text {th }}$ century BC. ${ }^{230}$ The appearance of these fibulae at the cemeteries of the eastern Carpathian Basin is dated by Kemenczei to the 'early Scythian' horizon, after

226 VAsIĆ 1999, 65-71 and Pls. 33/435-38/532; for their distribution: Pl. 66. The bow can be round or octagonal in cross section. The foot can be decorated in the middle with a $\mathrm{V}$ motif or vertical lines, and sometimes with concentric circle motifs or tremolo lines.

227 Čović 1987b, 602-604, 608, 612, 618 and Pls. LXI/13, 16; LXII/5, 9. - VAsić 1999, 69. - Gavranović 2011a, 190-191 and Fig. 174. Younger finds no longer have the bow with polygonal section. The closing of the cut-out on the foot took place at the end of phase IVc-1. On their appearance at Glasinac as early as the end of the $8^{\text {th }}$ century BC, and on their classification into three variants: LUCENTINI 1981, 72-73 and Figs. 1/20-22; 1-2.

228 BADER 1983, 85-90 and Pls. 29/225-229; 30/230-234A; 31/236238; for their distribution: Pl. 49.

229 Gergova 1987, 44-53 and Pls. 14-17. - Fibulae of type B II 2 developed from those with the foot in the form of an hourglass of type B II 1.

230 Gergova 1987, 50-51 and Pl. 17/211-215. 
the middle of the $7^{\text {th }}$ century BC, pointing out the parallels with the finds from Donja Dolina. On the other hand, he dates the appearance of this type of fibula in the northern and central Balkans as early as the first half of the $7^{\text {th }}$ century BC..$^{231}$ Also, the appearance of these fibulae, distributed from the Aegean to the Carpathian Basin and the southeast Alps, is dated by Teržan to the first half or the middle of the $7^{\text {th }}$ century BC (at the latest) until the early $6^{\text {th }}$ century BC. In the area of the central Balkans, these fibulae are items of female costume, and often come in pairs, ${ }^{232}$ as is the case with some of the graves from Donja Dolina.

The fibulae from Donja Dolina are most often decorated with the $\mathrm{V}$ motif on the foot, which is a characteristic of the fibulae of variant 2, which include the fibulae from grave N. Čegrlja 3 and those from graves N. Šokić I 8 and $31 .{ }^{233}$ This motif on the foot is most widespread in the area from eastern Bosnia to northwest Bulgaria and Macedonia, already appearing during the second half of the $7^{\text {th }}$ century BC, which applies to the tremolo decoration too. ${ }^{234}$ The tremolo decoration along the edge of the foot, as seen on the fibula from grave N. Čegrlja 3, is known on the fibula from the Grivac which belongs to the variant $2 \mathrm{a}$ with a very low foot and oval cut-outs, but this fibula has a $\mathrm{V}$ motif in the middle part. ${ }^{235}$

Thus, double-loop bow fibulae with the foot in the shape of a Boeotian shield from the graves in Donja Dolina have parallels in the area of Glasinac, but a pair of fibulae from grave M. Petrović Jr. 6 (Fig. 4/3-4) has direct parallels in the finds from northern Bulgaria; along with the parallels documented by the analysis of open-work belts, this testifies about direct contacts along the rivers Sava and Danube. This form of fibula was a characteristic item of female costume in Donja Dolina, where they have been found in

231 Kemenczei 2004, 79, 81, 83, 98 and Fig. 1/2, 5-6. - On that occasion, he dated grave N. Čegrlja 3 to the middle of the $7^{\text {th }}$ century BC. 232 TeržAn 1987a, 15 and Fig. 9/7. - TeržAn 1987b, 420. - TeržAn 1990, 82, 99-100, 139. - MetZner-Nebelsick 2002, 110-111, 415, 426 and Fig. 39/3. - Gavranović 2011a, 190. - Heilmann 2016, 16. 233 Heilmann 2016, 21-22 and Fig. 3. - The fibulae of variant 2 are distributed across the central Balkans, in northwest Bulgaria and Macedonia from the middle of the $7^{\text {th }}$ to the first half of the $6^{\text {th }}$ century $\mathrm{BC}$, while the fibulae of variant 3 are dated to the end of the first half and the middle of the $6^{\text {th }}$ century $\mathrm{BC}$ and are restricted to the western Balkans.

234 VAsić 1999, 70. - Gavranović 2011a, 190 and Fig. 174/12-17. HeiLmann 2016, 12-13, 16, 18, 22 and Fig. 3. - The fibulae of variant 2 after the classification of Daniela Heilmann have a bow of round or polygonal cross section and with expansions on the terminals. The low and wide foot is decorated with an incised V motif and less frequently with the motif of relief ribs.

235 VASIĆ 1977, 33 and Pl. 23/1. - VASIĆ 1999, 66 and Pl. 34/451. HeILmann 2016, 15. graves which can be dated to the second half of the $7^{\text {th }}$ and the beginning of the $6^{\text {th }}$ century BC.

Presented as a distinct form, the fibula from inhumation grave 1 in the garden of N. Šokić was found on the chest of a richly equipped young woman along with a plate fibula, a boat-shaped fibula, and four other fibulae. It is a single-loop fibula with a rectangular symmetrical foot and a vertical rib along the middle, with the motif of a concentric circle below it. On each side of the rib there are two concentric circles. The bow has a low triangular cross section and is flat on the back. At the top of the bow there are five loops with hanging chains consisting of single and double small rings and with pendants hanging at the end of the chains. At the end of the bow, towards the missing spiral, there is a spherical protrusion. ${ }^{236}$

The closest parallel is a fibula from the Debele Brdo-Soukbunar site, which is a single-loop fibula with a large triangular foot and five loops at the top of the narrow crescent-shaped bow. The upper and lower edges of the bow are decorated with short oblique incisions. Chains hanging from the loops consist of small rings. Close to the spiral on the bow, there is also a pendant with one ring on each terminal. The fibula has been dated to the $8^{\text {th }}$ and $7^{\text {th }}$ centuries $\mathrm{BC} .{ }^{237}$ However, the only direct parallel is the pair of single-loop fibulae from grave 8 in tumulus I at the Bazje site in northern Albania. The fibulae have the foot in the shape of a Boeotian shield, with a vertical groove along the middle. The bow is crescent shaped; at its top there are five loops with hanging pendants of complex composition. ${ }^{238}$ On the basis of its double-loop fibulae with a rectangular symmetrical foot with two holes of the kind that was also found in Donja Dolina (phase 2c), the grave can be dated to phase Glasinac IVc-2. These fibulae are considered to be a further development of fibulae with the foot in the shape of a Boeotian shield; their appearance has been dated to the turn of the $7^{\text {th }}$ and $6^{\text {th }}$ centuries BC. Aside from the sites in eastern Bosnia and western Serbia, fibulae of this shape have also been found at sites in Herzegovina and northern Albania. ${ }^{239}$

236 Truhelka 1904, 84 and Fig. 65. - Marić 1964, Pl. XIII/34. Temple rings of type A were found in the grave.

237 Gavranović 2011b, 25 and Fig. 20/2.

238 Prendi 1976, 172 and Pl. VI/14. - Islami 1983, 115 and Fig. 8, Pl. IV/2-3.

239 Benac, Čović 1957, 41-42. - LuCentini 1981, 73-74 and Fig. 2/3-5. - Čović 1987a, 252 and Pl. XXVIII/4. - Čović 1987b, 604, 618-619 and Pl. LXII/24, 26, Fig. 36/20. - VAsić 1999, 71. Gavranović 2011a, 190-191, 199 and Fig. 174/18, 20. - These fibulae are usually between $3 \mathrm{~cm}$ and $5 \mathrm{~cm}$ long, with a trend of getting even smaller. 


\subsection{Plate Fibulae}

One of the most numerous forms of fibulae in the Donja Dolina is the plate fibula, which has often been found in graves with temple rings of types A and B (Tab. 4). In inhumation graves, they are placed on the chest of the deceased women, sometimes in pairs. This is testified to by grave N. Čegrlja 3 (Fig. 2) 240 $^{24}$ and grave 1 in the garden of N. Šokić. ${ }^{241}$ There are two distinct basic types, dominated by fibulae that are richly decorated with hammered and open-work decorations, while the pin is fastened on the back (Figs. 5/10, 7/11). ${ }^{242}$ Only grave N. Šokić II 4 included a plate fibula with eight petals (Fig. 9/4). ${ }^{243}$ Plate fibulae have been found in a few other graves in Donja Dolina, which also contain some other forms of temple rings, most often the variants of type D. ${ }^{244}$ We should add the chance finds of plate fibulae from the cemetery and one from Gradina. ${ }^{245}$

The plate fibulae from the cemetery in Donja Dolina are dated to phases $2 \mathrm{a}$ and $2 \mathrm{~b}$; the youngest finds date to phases $2 c$ and $3 a-1$, when they disappear. ${ }^{246}$ These fibulae have been singled out as the Donja Dolina type and they are dated in the $7^{\text {th }}$ and $6^{\text {th }}$ centuries BC. One of the oldest finds is a rosette-shaped fibula from grave N. Šokić II 4 (Fig. 9/4), where it was found together with objects originating from the eastern Balkans and Greece. Plate fibulae are widespread from Hallstatt to Asia Minor, but their places of origin are northern Greece and Albania, from where they reached Donja Dolina through Glasinac. ${ }^{247}$ Plate fibulae are analysed in detail by Teržan, who distinguished two variants: fibulae with a spherical plate and fibulae with a rosette-shaped plate, which she dates from the second half of the $8^{\text {th }}$ to the middle of the $6^{\text {th }}$ century BC. As for the finds from Donja

240 Truhelka 1904, 85 and Pl. XL/3.

241 Truhelka 1904, 84 and Fig. 65.

242 Grave M. Petrović Jr. 3: Truhelka 1904, 90. - Grave M. Petrović 2: Truhelka 1904, 118. - Gavranović 2011b, 128 and Fig. 158/12. - Grave M. Petrović 3: Truhelka 1904, 119. - Čović 1987a, 246 and Pl. XXVI/4-5. - Grave M. Petrović Sen. 9: Truhelka 1904, 124 and Pl. LXXI/1.

243 Truhelka 1904, 133 and Pl. LXXVII/19. - This grave has been dated to the oldest burial horizon from Ha C1: TERŽAn 1974, 43 and Fig. 7. Also: Kilian 1975, 51, 98, 108 and Pls. 28/12; 82/1.

244 Grave M. Petrović Jr. 35: Truhelka 1904, 98 and Pl. XLVIII/17-18. - Grave N. Šokić I 13: Truhelka 1904, 114 and Pl. LXII/7. - Grave N. Šokić I 26: Truhelka 1904, 115 and Pl. LXV/6. - Grave M. Petrović Sen. 16: Truhelka 1904, 126. - Gavranović 2011b, 129 and Fig. 160/11. - Grave N. Šokić II 5: Truhelka 1904, 134 and Pl. LXXVII/22.

245 Truhelka 1904, 144 and Fig. 83. - Marić 1964, Pls. IX/18; $\mathrm{XIII} / 6$.

246 Čović 1987a, 241, 246, 248, 252 and Figs. 15/13; 16/1, Pls. XXVI/5; XXVII/13.

247 Gavranović 2011a, 192 and Fig. 178.
Dolina, which include both variants, she dates them from the middle of the $7^{\text {th }}$ to the $6^{\text {th }}$ century BC. Plate fibulae are widespread in the Aegean too, where they originated, and in the Balkans, all the way to the southeastern Alps. They have also been found in the cemeteries of Ferigile and Szentes-Vekerzug; in fact, the fibulae from the latter site, which have a star-shaped open-work decoration, have been recognized as the closest parallel to those from Donja Dolina, dated to the second half of the $7^{\text {th }}$ century BC. On the other hand, it is pointed out that rosette-shaped plate fibulae have been found at sites ranging from the southern Balkans to Donja Dolina. ${ }^{248}$ As pointed out, they have been found in a cremation grave from tumulus 45 in the Ferigile cemetery, with parallels sought in Donja Dolina, ${ }^{249}$ especially for those from the Szentes-Vekerzug cemetery, dated to the first half of the $6^{\text {th }}$ century BC and reflecting the influence of the Hallstatt-Balkans fashion of wearing fibulae. ${ }^{250}$

Thus, the plate fibulae from the Donja Dolina cemetery indicate a distinctive and favourite form of female costume originating in the southern Balkans, which arrived in this area around the middle of the $7^{\text {th }}$ century $\mathrm{BC}$ and remained in use until the second half of the $6^{\text {th }}$ century BC.

\subsection{Spectacle Fibulae}

In the Donja Dolina cemetery and in some cemeteries of the Ciumbrud group with type A temple rings, small bronze spectacle fibulae without a figure-of-eight loop in the middle part (Tabs. 2, 4) have been found, singly or in pairs. In grave N. Čegrlja 3, on the right side of the chest of the woman, there was a small fibula of this shape (Fig. 2/10). ${ }^{251}$ Fibulae of this kind have also been found in the Donja Dolina cemetery in grave N. Šokić $1,{ }^{252}$ and there were two fibulae in grave M. Petrović 9. ${ }^{253}$ Chance finds from Donja Dolina include another three fibulae of this kind. ${ }^{254}$ In grave XIV of the Ciumbrud-Podireu cemetery, there was a small bronze

248 TERŽAN 1974, 43 and Fig. 7. - TERŽAN 1987b, 420, 425 and Fig. 4. - TeržAn 1990, 139, 141, 212 and Map 6. - TeržAn 1998, 515. - EgG 1996, 272-273 and Fig. 151.

249 BADER 1983, 105 and Pl. 34/309-310.

250 KemencZei 2004, 85, 87 and Figs. 5/2, 7, 16; 6/1-2. - TeržAN, Hellmuth, Heimann 2011, 268 and Fig. 12.

251 Truhelka 1904, 85 and Pl. XL/10.

252 Marić 1964, Pl. IX/7. - The older settlement at Donja Dolina included a fibula with a figure-of-eight loop (MARIć 1964, Pl. IX/6). The fibula belongs to the Haslau-Regelsbrunn type and has been dated between $\mathrm{Ha} \mathrm{B} 3$ and $\mathrm{Ha} \mathrm{C} 1$, even though it remained in the western Balkans until the $5^{\text {th }}$ century BC: Gavranović 2011a, 183 and Fig. 172/1. - РАвST 2012, 379.

253 Truhelka 1904, 121.

254 Truhelka 1904, 143 and Fig. 82, Pl. LXXXI/1. 
fibula on the chest of the woman, ${ }^{255}$ while grave 7 from the Cristești-Szörtsey cemetery contained two fibulae of this shape. ${ }^{256}$

The small spectacle fibulae without a figure-of-eight loop are associated with phase $2 a, 257$ distinguished as the Santa Lucia type, and dated to the developed $7^{\text {th }}$ century BC..$^{258}$ At Glasinac they appear in phase IVb and are considered to be a form that was inherited from the previous phase, but they are frequent in and characteristic for phase IVc1 , in which the bottom side had a bronze or (more often) iron frame with a pin and a hook-shaped foot. This is one of the more frequent forms in phase IVc-2 too. ${ }^{259}$ Teržan also defines them as fibulae of the Santa Lucia type, which already appears in hoards of the older Urnfield Culture; in the central Balkans they belong to the female costume starting from the Glasinac IV phase. ${ }^{260}$ A similar opinion is provided by Bader, who points out that the spirals consist of four to eight coils of wire of round or rectangular cross section, and are found on the shoulder or chest in the graves, sometimes in pairs. Along with the already mentioned graves of the Ciumbrud group, a pair of small spectacle fibulae was found in grave 15 in the Ozd cemetery. The fibulae are distributed from the southeastern Alpine area, across Donja Dolina, Glasinac, and western Serbia, to Poland in the north and Greece in the south. Finds thereof have been recorded starting from hoards of the older Urnfield phase (Ha A1) to $\mathrm{Ha} \mathrm{D}$; the older ones are noticeably larger, while smaller ones have been found in the graves dated to the beginning of $\mathrm{Ha} \mathrm{D}$, such as the Ciumbrud group cemeteries. ${ }^{261}$ Gergova distinguishes them as type $\mathrm{C} 2$; they are few in number in the area of Bulgaria, and she dates them from the $9^{\text {th }}$ to

255 Ferenczi 1965, 102 and Fig. 8/16. - Ferenczi 1966, 54 and Fig. 18/2. - FerenCZi 1969, 50 and Fig. 18. - Bader 1983, 57 and Pl. 11/89. - Kemenczei 2004, 83 and Fig. 3/7. - Also, child grave VI contained a fragment of a fibula of this type: BADER 1983, 56 and Pl. $11 / 88$.

256 Crişan 1965a, 59 and Fig. 12/6. - CRIŞAn 1974, 107 and Fig. 6/2. - Bader 1983, 57 and Pls. 11/90; 56/B1. - KemencZei 2004, 83 and Fig. 3/10.

257 Čović 1987a, 241, 243.

258 Gavranović 2011a, 184.

259 Čović 1987b, 594, 607, 618 and Pls. LX/21; LXI/17; LXII/23. VAsić 1999, 34. - Also: Lucentini 1981, 69 and Fig. 1/1-2.

260 TerŽAn 1987a, 10 and Fig. 6/4-5.

261 BADER 1983, 56-60 and Pls. 11-13/86; for their distribution: Pl. 44. - Grave 15 from the Ozd cemetery: BADER 1983, 57 and Pls. 11/92-93; 56/C1-2. - VAsiliev, ZRíNYI 1974, 96, 135 and Pl. XVI. KemenCZei 2004, 83. - Kemenczei points out that spectacle fibulae without a figure-of-eight loop appear in the eastern Carpathian Basin in the 'first Scythian horizon', and that they might be a local tradition or originating from the northern Balkans: KeMENCZEI 2004, 85. the $7^{\text {th }}$ century BC. ${ }^{262}$ The Santa Lucia-type fibulae from the sites in Serbia are analysed by Vasić, who points out that they appear over the entire duration of the Early Iron Age, and concludes that the younger forms are smaller. The fibulae occasionally have an iron pin. They have been found most often in graves, singly or in pairs, dated from the $8^{\text {th }}$ to the $5^{\text {th }}$ century BC, and widespread across central Europe, the Balkans, and Italy. ${ }^{263}$ The last detailed analysis of spectacle fibulae was carried out by Sabine Pabst, who also distinguishes the single-piece Santa Lucia type, characterized by the lack of the figure-of-eight loop in the middle, and made from a wire of rounded cross section. It is a widespread form, which she associates with the mentioned finds from the graves of the Ciumbrud group. Santa Lucia-type fibulae appear as early as the $10^{\text {th }}$ century BC; they are present in the $9^{\text {th }}$ century BC in the central Balkans and after $700 \mathrm{BC}$ at Glasinac and in the northern part of the central Balkans. ${ }^{264}$ On the other hand, multi-part spectacle fibulae without a figure-of-eight loop, mostly of small dimensions, are defined by her as the Glasinac type; she distinguishes three variants on the basis of the material they are made of. They are considered characteristic for the costume of adult women and girls. The fibulae of this type are distributed at Glasinac, in western Serbia, and in the Drina valley, with Donja Dolina being the northernmost site. At Glasinac, they appear in the graves of phase IVc, i.e. in the $7^{\text {th }}$ and the first half of the $6^{\text {th }}$ century BC. ${ }^{265}$

Thus, small bronze spectacle fibulae from the Donja Dolina cemetery and the Ciumbrud group cemeteries indicate that it was a female costume item worn from the middle of the $7^{\text {th }}$ to the first half of the $6^{\text {th }}$ century BC.

\subsection{The Marvinci-Gogoșu-Type Fibulae}

One of the distinctive forms of fibulae in the Donja Dolina cemetery is the single-loop bow fibula with a rectangular foot of the Marvinci-Gogoșu type. ${ }^{266}$ Two fibulae of this type have been found in inhumation grave M. Petrović 1 together

262 Gergova 1987, 53-54.

263 VAsić 1999, 30, 34-35 and Pls. 8-12/103-175; for their distribution: Pl. 62A.

264 Равsт 2012, 88, 91, 255 and Fig. 8/1, Pl. 15/2; 392-393 and List 33, Map 25. - They remained on the Santa Lucia cemetery as long as the $5^{\text {th }}$ century BC.

265 Равsт 2012, 105-106, 172-173 and Fig. 11/1, Pl. 16/1-3; 396397 and List 45, Map 30/2. - It is assumed that the fibula from grave N. Čegrlja 3 belongs to the Glasinac type.

266 Kilian 1975, 73-74, 97-98 and Pl. 85/1-2. - PARZinger 1992, 234 and Fig. 10/4. - From northern Greece to southern Romania, from the Morava and the Vardar to the Struma and the Iskar in the east. Rare further west, as in Donja Dolina. 
with type B temple rings (Tab. 4). The better-preserved fibula has the saddle-shaped upper part of the low rectangular foot and ends with a button. ${ }^{267}$ In the Donja Dolina cemetery fibulae of this type have also been found as a pair in grave N. Šokić I 1, ${ }^{268}$ while grave M. Petrović Sen. 3 contained a single fibula. ${ }^{269}$ We should also mention the Marvinci-Gogoșu-type fibula from the grave in tumulus XXVIII in the eponymous cemetery, together with a small temple ring. ${ }^{270}$

Fibulae of this kind are dated to phase $2 b,{ }^{271}$ while Vasić places them in horizon $2 .{ }^{272} \mathrm{He}$ then classifies bow fibulae with a rectangular foot into several variants, one of which is represented by fibulae with a large horizontal foot, which he calls the Marvinci-Gogoșu type. They are characterized by a smooth, rounded cross section to the bow with button-shaped expansions on the terminals and a square or rectangular foot with a saddle-shaped upper part and a button at the end. The fibulae of this type are distributed in the greatest number in the central Balkans, but they have also been found from Thessaly to Transylvania and Donja Dolina. It is assumed that this form originated in Chalcidice at the end of the $7^{\text {th }}$ century BC, spread to the Danube at the beginning of the $6^{\text {th }}$ century BC, and remained in use until the beginning of the $5^{\text {th }}$ century BC. For the fibulae from Donja Dolina, it is pointed out that they are typologically related to those from the sites in northwestern Bulgaria and southwestern Romania, from where they probably arrived, and have been dated to the beginning of the $6^{\text {th }}$ century BC. ${ }^{273}$ On the other hand, the fibulae of the Marvinci-Gogoșu type, also called the Donja Dolina type by Teržan, are dated by her from the middle of the $7^{\text {th }}$ century BC onwards. ${ }^{274}$ Actually, these single-loop bow fibulae with a square or rectangular foot were first distinguished as the Donja Dolina type by Bader. The bow has a semicircular outline and a varied cross section, while the foot can be decorated or undecorated, and as a rule, ends with a button. The highest number of these fibulae comes from

267 Truhelka 1904, 117-118. - Marić 1964, Pl. IX/33-34.

268 Truhelka 1904, 110-111 and Pls. LVIII/4; LIX/7.

269 Truhelka 1904, 122 and Pl. LXVIII/9.

270 Berciu, Comşa 1956, 425, 428 and Fig. 151/2. - Bader 1983, 94 and $\mathrm{Pl}$. 32/262.

271 Čović 1987a, 246, 248 and Pl. XXVI/2.

272 Vasić 1977, 18 and Pls. 23/2; 51/3-4. - Sinjac Polje: KaPuran, Blagojević, BizjaK 2015, Figs. 6/2; 9/5.

273 VAsić 1987a, 42-50 and Pl. 1/3-4, App. 1. - VAsić 1999, 71-77 and Pls. 40-41/553-581; for their distribution: Pl. 67A. The Marvinci variant is present in Greek Macedonia and North Macedonia, Serbia, and northwest Bulgaria; and the Gogoşu variant with a decorated bow and foot, in Bulgaria and Oltenia.

274 TERŽAn 1987a, 19. - TERŽAN 1990, 74. female graves; in most cases, they are worn singly, and they are rarely found in pairs. The finds from inhumation graves show that the deceased wore them on the left side of the chest or shoulders. The fibulae have been dated from the middle of the $7^{\text {th }}$ to the middle of the $6^{\text {th }}$ century BC, and not later than the second half of the $6^{\text {th }}$ century BC..$^{275}$ The Marvinci-Gogoșu-type fibulae are distinguished by Gergova as type A III 4 with two variants, where variant $\alpha$ is the one with a bow that has no decorations except ring-like expansions, and the button from the remains of the foot can be separated by a rib. The fibulae of this variant are distributed in northwest Bulgaria, Romania, and Macedonia, and are dated to the $7^{\text {th }}$ and $6^{\text {th }}$ centuries BC, with a mention of the finds from Donja Dolina. Variant $\beta$ is characterized by a massive bow, profiled with narrow and thick transverse ribs and somewhat wider ribs; the foot is low and is less than one half of the height of the fibula. These fibulae are considered to be characteristic for the Gogoșu cemetery, meaning that it is a local variant that has been dated like the previous one. ${ }^{276}$ In his analysis of the fibulae from Donja Dolina, Gavranović also points out that the decorations of transverse grooves and tremolo lines on the foot along the edges are similar to the finds from the area of southwest Romania. Considering the combination of fibulae in grave N. Šokić I 1, this grave has been dated to the end of the $7^{\text {th }}$ and the first half of the $6^{\text {th }}$ century BC, while grave M. Petrović 1 has been dated to the early $6^{\text {th }}$ century BC. The fibulae of this type again point to the importance of communication along the Sava and the Danube. ${ }^{277}$ The Marvinci-Gogoșu-type fibulae from Vekerzug group cemeteries are mentioned by Kemenczei. The fibula from cremation grave 61 from the Szentes-Vekerzug cemetery has a higher square foot ending with a conical button, while there are expansions at the end of the high bow towards the foot. The fibula actually belongs to the Novi Pazar type, a younger type from the end of the $6^{\text {th }}$ and the first half of the $5^{\text {th }}$ century BC. Finally, grave 23 in the Tiszavasvári-Dózsa-telep cemetery included a fragment of a fibula with a low foot ending with a button, which could belong to the Marvinci-Gogoșu type. ${ }^{278}$

Thus, bronze single-loop bow fibulae of the Marvinci-Gogoșu type can be classified into different variants on

275 BADER 1983, 93-99 and Pl. 50. The classification into variants depends on the cross section of the bow. A variant $E$ fibula was found in grave 1 from Simeria with temple rings with conical terminals (BADER $1983,96)$. Some of the described fibulae actually belong to the younger type of Novi Pazar.

276 Gergova 1987, 33-34 and Pls. 6/83-84; 7/85-88; 7/89-91.

277 Gavranović 2011a, 198 and Fig. 185.

278 KemencZei 2004, 87, 89-90 and Figs. 6/5-6; 7/1. 
the basis of the shape of the bow and foot, with regional and chronological differences. The fibulae from the graves in Donja Dolina can be dated to the second half of the $7^{\text {th }}$ and the beginning of the $6^{\text {th }}$ century BC, and point to contacts with eastern areas along the Sava and the Danube, primarily with the areas of southwest Romania and northwest Bulgaria.

\subsection{Poiana-Type Fibula}

Grave 20 in the Gîmbaș-Măgauricea cemetery, containing type A temple rings, also included a single-loop fibula with a tall undecorated trapezoidal foot and a bent prong of the Poiana type. ${ }^{279}$ The fibulae of this type were classified into two variants by Bader on the basis of the bow cross section; he classified the fibula from the Gîmbaș grave as variant $B$ with a triangular cross section to the bow with four deep and wide grooves in the middle and two more on the ends of the bow. Their origin is the Aegean area; they have been found in female graves and are distributed in southern Moldova and southern Transylvania, Bulgaria, and Macedonia. Their appearance has been dated from the beginning of the $7^{\text {th }}$ to the end of the $6^{\text {th }}$ century BC, while the Gîmbaş fibula has been dated to the turn of the $7^{\text {th }}$ and $6^{\text {th }}$ centuries BC. ${ }^{280}$ The fibulae of this kind, with a tall trapezoidal foot, have been distinguished by Gergova as type A II 3, variant $\gamma$, usually with a small and smooth bow and a group of three transverse ribs of different widths only in the middle. The back of the bow is smooth, while the tall foot has hammered protrusions. They are distributed in southern Thracia, but they also appear in Romania; they have been dated to the $7^{\text {th }}$ and $6^{\text {th }}$ centuries BC. ${ }^{281}$ They are also called Thracian fibulae, known from sites ranging from Greece to Transylvania and Moldova. ${ }^{282}$ In her last analysis, Teržan dates the Poiana-type fibulae from the middle of the $8^{\text {th }}$ to the late $7^{\text {th }}$ century BC, distinguishing the Poštela variant, which is present in the southeastern Alpine area, as a local product. The Poiana-type fibulae are classified into variants according to the shape of the bow and foot; they are distributed across Transylvania, Moldova, and Bulgaria, pointing to the local female costume of the eastern Balkans and having their origin in Thracia or the Aegean, where they were worn from the $10^{\text {th }}$ to the $8^{\text {th }}$ century BC. The fibula from the Gîmbaș

279 Bader 1983, 99 and Pl. 34/294. - Vulpe 1984, 47 and Fig. 8/8. - Kemenczei 2004, 90 and Fig. 4/23. - TeržAn 2016, 340 and Fig. $3 / 2-4$.

280 BADER 1983, 99-101 and Pls. 33/292-293; 34/294-296; for their distribution: Pl. 51.

281 Gergova 1987, 29-30 and Pls. 4/59-60; 5/61-68; 6/69-71.

282 Kilian 1975, 108 and Pl. 81. - Parzinger 1992, 234 and Fig. 9/3. grave is dated by her to the late $7^{\text {th }}$ early $6^{\text {th }}$ century BC, as one of the youngest finds. ${ }^{283}$

\subsection{Boat-Shaped Fibulae}

The important finds for the dating of the Donja Dolina graves with temple rings of types $\mathrm{A}$ and $\mathrm{B}$ are different forms of boat-shaped fibulae (Tab. 4) belonging to the defined types, the most numerous ones being of the Šmarjeta type. $^{284}$

The boat-shaped fibulae of the Šmarjeta type, with three longitudinal ribs with transversal incisions (one rib along the middle of the bow and one along each edge) and ribs on both ends of the bow, have been found in grave M. Petrović 3 together with temple rings of types $A$ and $C 1,285$ but also in some other graves in Donja Dolina. ${ }^{286} \mathrm{~A}$ boat-shaped fibula with transverse fields with a netting decoration comes from grave N. Šokić II $5,{ }^{287}$ while two fibulae have been found at the Gradina settlement. ${ }^{288}$ Another fibula from the cemetery area is a boat-shaped fibula with a bow decorated with groups of transverse grooves. ${ }^{289}$ Boat-shaped fibulae of somewhat different forms have been found in some other graves with temple rings of types A and B. Grave M. Petrović Jr. 3 contained a boat-shaped fibula with two transverse grooves on the ends of the bow, while the pin seems to have been made of iron and fastened to the end of the bow (Fig. 3/4). ${ }^{290}$ This fibula is similar to another fibula from the cemetery. ${ }^{291}$ Grave M. Petrović 8 seems to contain two boatshaped fibulae, while the other two fibulae were close to the Sanguisuga type with a rhombic cross section to the bow. One boat-shaped fibula has a long foot, the ends of the bow

283 TeržAn 1990, 59 and Map 5. - TeržAn 2016, 338-344 and Figs. $1 / 1-2 ; 5$.

284 For some boat-shaped fibulae, considering the publications until now, it is impossible to determine the exact shape, e.g. for grave 1 from the garden of N. Šokić, there is mention of a small boat-shaped fibula and another four fibulae of an unknown type: TruHelka 1904, 84 and Fig. 65.

285 Truhelka 1904, 119. - Gavranović 2011a, 193 and Fig. 179/1. 286 Grave M. Petrović Jr. 51: Truhelka 1904, 102 and Pl. XLI/8. - Grave N. Šokić I 1: Truhelka 1904, 110 and Pl. LVIII/6. Grave I. Stipančević 8: Truhelka 1904, 128 and Pl. LXXV/9.

287 Truhelka 1904, 134 and Pl. LXXVII/29.

288 Truhelka 1904, 70 and Fig. 43. - Gavranović 2011b, 81 and Fig. 103/5-6.

289 Marić 1964, Pl. IX/21. - Gavranović 2011a, 193 and Fig. 179/5. 290 Truhelka 1904, 90 and Pl. XLII/19. - Grahek 2004, 142, 144, associates this fibula with type $2 \mathrm{~d}$, characterized by a single longitudinal rib in the middle of the bow. These fibulae developed from the

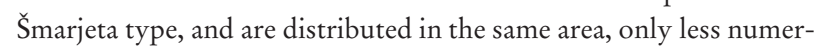
ous (Graher 2004, Fig. 34). They also date to the Stična 2 subphase, and still remain within the serpentine fibula horizon.

291 Čović 1987a, 246 and Pl. XXVI/3. 
having several groups of transverse grooves, and longitudinal ribs in the middle. The other fibula also has a long foot and a bow with dense transverse ribs, and is associated with the late $7^{\text {th }}$ and the early $6^{\text {th }}$ century BC. ${ }^{292}$ Grave M. Petrovic Sen. 16 included a boat-shaped fibula with a long foot ending with a knob. At each end of the bow there is a group of transverse grooves. It is followed by a smooth part towards the middle of the bow and, again, a group of narrow transverse grooves on each side, while the central part of the bow has thick longitudinal ribs. ${ }^{293}$ The fibula from grave M. Petrović Jr. 3 is similar to one found in the settlement, which also has transverse grooves at the ends of the bow, while the central part of the bow has longitudinal grooves. ${ }^{294}$

Grave M. Petrović Jr. 3 with a boat-shaped fibula, which also contains two amber-coated fibulae, is included by Teržan in the horizon matching subphase Stična 2 in the Dolenjska region. ${ }^{295}$ The boat-shaped fibulae from Donja Dolina are dated in the same way by Čović, who classifies them into phase 2 b. ${ }^{296}$ Boat-shaped fibulae of different types from the Donja Dolina cemetery are analysed in detail by Gavranović. The Šmarjeta-type fibulae, distributed from the southeastern Alps to Slovakia, are dated to horizon $\mathrm{Ha}$ C2-D1, i.e. to the late $7^{\text {th }}$ and the early $6^{\text {th }}$ century BC. They are similar to one of the fibulae from grave M. Petrović 8 , with several longitudinal ribs, and the one from the settlement, which could be a local product. There are also a number of boat-shaped fibulae with transverse groups with a netting decoration, such as the one found in grave N. Šokić II 5, dated to the late $7^{\text {th }}$ and the early $6^{\text {th }}$ century BC; they can include chance finds and a fibula decorated with thick transverse grooves distributed in groups. The fibula from grave M. Petrović Jr. 3, and the fibula outside the grave assemblage, are classified as the Gogoșu variant. The fibula from grave M. Petrović Sen. 16 has been dated on the basis of the other finds from the grave to the early $6^{\text {th }}$ century BC. ${ }^{297}$ The detailed analysis of the boat-shaped fibulae from Magdalenska gora distinguishes several types

292 Marić 1964, Pl. IX/25-26. - Gavranović 2011a, 193 and Fig. 179/2. - The grave also contains two fibulae close to the Sanguisuga type with a bow of rhombic cross section and a long foot: GAvRANOVIĆ 2011a, 196 and Fig. 183/1.

293 Truhelka 1904, 126. - Čović 1987a, 248 and Pl. XXVII/9.

294 Marić 1964, Pl. IX/23. - Gavranović 2011b, 81 and Fig. 103/7. - Graher 2004, 142 associates this fibula with the Šmarjeta type. 295 TeržAn 1974, 40, 42-43 and Fig. 7.

296 Čović 1987a, 248-249 and Pls. XXVI/3; XXVII/9.

297 Gavranović 2011a, 193-194 and Fig. 179. - Boat-shaped fibulae with a long foot and profiled knob at the end are most frequent in the areas of the Dolenjska and Sveta Lucija groups, where they were made after Italic models: GABRovec 1987, 49. with variants matching the specific finds of boat-shaped fibulae from Donja Dolina. ${ }^{298}$

The boat-shaped fibulae from Donja Dolina originate from the Dolenjska region, where such fibulae are characteristic for phase Stična, reaching their peak during subphase Stična 2, when they appear in richly furnished female graves. The boat-shaped fibulae of the Šmarjeta type, like those with transverse ribs, are most frequent in the Dolenjska region, but there is a noticeable spread toward the east. ${ }^{299}$ Boat-shaped fibulae of the Šmarjeta type are analysed in detail by Teržan, who says that they originated from the southeastern Alpine area, but they have been found at sites in western Hungary, southwestern Slovakia, and as far as Moravia and Donja Dolina. They have been dated to subphase Stična 2, i.e. the late $7^{\text {th }}$ and the early $6^{\text {th }}$ century BC. ${ }^{300}$ Regarding Šmarjeta-type fibulae, Parzinger concludes that those with a shorter foot and without a knob at the end are older, belonging to the second half of the $7^{\text {th }}$ century BC ( $\mathrm{Ha} \mathrm{C} 2)$, while those with a longer foot and a knob at the end still belong to the beginning of $\mathrm{Ha} \mathrm{D} 1$, i.e. to the early $6^{\text {th }}$ century BC..$^{301}$ Also, there is the interesting claim that among the Šmarjeta-type fibulae, which can be classified into variants according to the number of longitudinal ribs on the bow, those with three to five ribs (variant A) are found in greater numbers at the sites located further away from their basic distribution area. ${ }^{302}$ Boat-shaped fibulae of the Šmarjeta type are very rarely found further east, so it is suggested that they arrived from the southeastern Alpine area through Donja Dolina. ${ }^{303}$ The same conclusion would probably apply to the chance finds of different forms of boat-shaped

298 Tecco-Hvala 2012, 208-216, 268, 348 and Fig. 81. - The fibula from grave M. Petrović 3 has its closest parallel in one of the fibulae of type $1 \mathrm{a}$, while the existence of the iron pin has parallels in type $1 \mathrm{~b}$ fibulae that are characteristic for the Dolenjska region. One of the fibulae from grave M. Petrović 8 could belong to type $6 \mathrm{~b}$, which has a bow with a ribbed surface.

299 Gabrovec 1987, 42, 48-49 and Fig. 3/9-10. - Dular 2003, 118, 130. - Gabrovec 2010, 52. - As the oldest variant from the Stična 1 subphase, a large boat-shaped fibula was singled out, with a long foot and a bow decorated with an incised ornament consisting of groups that are alternately decorated with a zig-zag line. Also: GrAHEK 2004, 142 and Fig. 35. - There are no such fibulae in Donja Dolina.

300 TeržAn 1974, 40, 42. - TeržAn 1987a, 19 and Fig. 13. - TeržAn 1990, 43, 101-102, 139, 181 and Map 16. - TeržAn 1998, 521, 524 and Fig. 4. - Also: Fenete 1973, 342-343. - PArzinger 1992, 233 and Fig. 9/1. - Glunz 1997, 98- 99 and Map 24. - Dular 2003, 118 and Fig. 70/6. - GraheK 2004, 142 and Fig. 33. - Kruh 2010, 110 and Pl. 126/1. - Tecco-Hvala 2012, 208, 216, 348 and Fig. 81/10. - Felcan 2018, 61 and Fig. 8.

301 Parzinger 1995, 16, 19, 179 and Fig. 7.

302 Glunz 1997, 98-99 and Map 24.

303 VAsIĆ 1999, 88 and Pls. 43/658; 44/660. 
fibulae that come from the sites in Romania, but also for some other forms of fibulae that are characteristic for the southeastern Alpine area (e.g. the fibula a tre bottoni from tumulus XXXV in Gogoșu). ${ }^{304}$ These are probably imports from the west that could have arrived across western Hungary or through communication along the Sava and the Danube, where the settlement of Donja Dolina played an important role. This is testified to by the aforementioned finds of specific forms of belts or specific types of fibulae, which certainly originated in the Danube area.

Another well-represented type of boat-shaped fibula in the Donja Dolina cemetery consists of fibulae with a lower bow of angular outline, decorated with transverse fields with a netting decoration, such as the one found in grave N. Šokić II 5, while two fibulae were found at Gradina. The fibulae of this kind, classified as type 6 a, are dated to the Stična 2 subphase and are distributed in the southeastern Alpine area, especially the Dolenjska region, but also across western Hungary all the way to Slovakia and Moravia in the north. ${ }^{305}$ The bow of one fibula from the cemetery is decorated with thick groups of transverse grooves; these fibulae are classified in the Dolenjska region as type $1 \mathrm{~b}$ fibulae. ${ }^{306}$

Different forms of boat-shaped fibulae from the Donja Dolina cemetery represent one of the more numerous, but also more heterogeneous groups of fibulae that certainly originate from the southeastern Alpine area, where they were created after Italic models. In fact, the forms that came to Donja Dolina, such as the Šmarjeta-type fibulae or fibulae with transverse groups with netting decoration, are characteristic for the area of Dolenjska during the Stična 2 subphase.

\subsection{Amber-Coated Fibulae}

Along with boat-shaped and plate fibulae and different forms of temple rings (Tab. 4), grave M. Petrović Jr. 3 contained two amber-coated fibulae with a bronze wire bow, where both ends of the bow still have narrow ring-like expansions (Fig. 3/3). ${ }^{307}$ These fibulae are dated to phase $2 b,{ }^{308}$

304 BADER 1983, 108-115 and Pls. 35-36/345-350; for their distribution: Pl. 61D. - Of particular importance is the find of a boat-shaped fibula, singled out as the Gogoşu type, with the bow decorated with five groups of transverse ribs from the inhumation grave in tumulus XLVIII, where it was found together with a Marvinci-Gogoşu type fibula.

305 TeržAn 1990, 101-102, 158 and Map 15. - Fekete 1973, 342, 348. - Glunz 1997, 102 and Map 25. - Dular 2003, 130 and Fig. 75/8. - Potrebica 2003, 219-221 and Map 2. - Grahek 2004, 140-142 and Fig. 32. - Gabrovec 2010, 52. - Tecco-Hvala 2012, 210 and Fig. 81/11. - EgG, Kramer 2016, 80-83, 203 and Map 35.

306 GraheK 2004, 140-142. - Tecco-Hvala 2012, 208, 214.

307 Truhelka 1904, 90 and Pl. XLII/16.

308 Čović 1987a, 246, 249 and Fig. 15/15. while Teržan dates this grave to the late $7^{\text {th }}$ and the early $6^{\text {th }}$ century BC. ${ }^{309}$

Fibulae coated with bone, glass, or amber are considered to be characteristic for subphase Stična 2 in the Dolenjska region where they are produced; their origin is northern Italy, where they appeared in the second half of the $7^{\text {th }}$ century BC. Good examples are the amber-coated and bone-coated fibulae in the princely female grave 27 of tumulus 48 in Stična, which still contained large boat-shaped fibulae. Along with some contemporary examples from the Dolenjska region, this testifies that coated fibulae appear in the graves of women and girls who had a prominent status in the community. ${ }^{310} \mathrm{~A}$ bone-coated fibula has been found in grave 2 of tumulus II in nearby Kaptol and is associated with horizon 3 of the Martijanec-Kaptol group, i.e. the fibulae have been dated to the end of the $7^{\text {th }}$ and the beginning of the $6^{\text {th }}$ century BC. ${ }^{311}$ It should be noted that the first analysis of the fibulae with different coatings - bone, amber, glass - on the wire bow of (usually) square or (rarely) rounded cross section was undertaken by Vida Starè. Amber-coated fibulae are frequent in tumuli at Vinji vrh and in the Hallstatt cemetery. As we pointed out, amber-coated fibulae have been dated to the late $\mathrm{Ha} \mathrm{C}$ and the beginning of $\mathrm{HaD}$, i.e. to the developed $7^{\text {th }}$ and the beginning of the $6^{\text {th }}$ century BC. ${ }^{312}$ In the last classification, the fibulae are classified into two types according to the bow outline, where the Donja Dolina fibulae belong to type $B$ with a square outline bow, which is distributed in northern Italy, the Soča valley, the Dolenjska region, and the Hallstatt cemetery, all the way to Donja Dolina. ${ }^{313}$

Thus, the amber-coated bow fibulae from grave M. Petrović Jr. 3 can be considered as an import from the area of Dolenjska, together with the boat-shaped fibula from the same grave, where they were a characteristic item of female costume in subphase Stična 2, i.e. in the late $7^{\text {th }}$ and the beginning of the $6^{\text {th }}$ century BC.

\subsection{Borajna-Type Fibulae}

One of the distinctive forms of bronze fibulae in the Donja Dolina cemetery consists of the Borajna-type fibulae (Tab. 4). They are small single-loop bow fibulae with a ribbed bow and a narrow and long asymmetrical triangular foot without a knob at the end. Such a fibula was found

309 TERŽAN 1974, 43. - TERŽAN 1990, 149. 310 Gabrovec 1987, 49-50 and Pl. V/2. - TeržAn 1987b, 40. Teržan 1990, 103, 149. - Dular 2003, 130 and Figs. 73/1; 75/5-7. - Gabrovec 2010, 52 and Pl. 18/20-21, 24. - Tecco-Hvala 2012, 226, 230, 268, 348 and Fig. 86/1.

311 Vinski-Gasparini 1987, 208 and Pl. XX/2.

312 STARÈ 1978, 64-76 and Pl. 1/1, 3-4, 6-8, Map 2-3.

313 Glunz 1997, 71-72 and Pl. 26/9-15, Map 12. 
together with several types of temple rings in grave M. Petrović Jr. 6 (Fig. 4/7). There is a ring-like thickening on each side of the bow and two ring-like thickenings in the middle of the bow. ${ }^{314}$ Another Borajna-type fibula has been found in grave N. Šokić II 2. The fibula has a ribbed bow with one transverse ring-like thickening in the middle and at the ends of the bow. The narrow triangular foot is decorated with oblique grooves. ${ }^{315}$ Fibulae of this type have been found in several other graves - burials of both women and men - with two graves standing out: the richly furnished female grave N. Šokić I 1 and the warrior grave M. Petrović Jr. 39.316

These fibulae have been dated to phase $2 \mathrm{~b}$ and are called the Glasinac knob fibulae. ${ }^{317}$ They appeared at Glasinac at the beginning of phase $\mathrm{IV}_{\mathrm{c}-2}$, but there remains the possibility that they already appeared at the end of the previous phase IVc-1. It is a heterogeneous group of fibulae, with noticeable differences in bow shape and decoration. ${ }^{318}$ Borajna-type fibulae are defined by Teržan, who identifies them as a Balkans concept of ribbed-bow fibulae that appeared at Glasinac and are characterized by a taller foot than the ribbed-bow fibulae appearing in the eastern Alpine area. Actually, both types appear in the Donja Dolina cemetery within the same graves (graves M. Petrović Jr. 39 and I. Stipančević 8), although the Borajna-type fibulae are probably older. Borajna-type fibulae are distributed from Glasinac and western Serbia to Donja Dolina and dated to the late $7^{\text {th }}$ continuing into the $6^{\text {th }}$ century BC. There are changes in the design of the foot, which eventually gets a knob, already turning into the Potpećine type at the beginning of the $6^{\text {th }}$ century BC. ${ }^{319}$ Borajna-type fibulae are described by Vasić within the heterogeneous group of bow fibulae with ring-like thickenings in the middle and at the bow ends, and with transverse grooves. The foot is triangular or trapezoidal and can be decorated with lines. Since it is often not known how the foot ends, or whether it has a knob at the end, using a different classification into variants on the basis of the number of thickenings in the middle of the bow has

314 Truhelka 1904, 91 and Pl. XLIII/15.

315 Truhelka 1904, 132 and Pl. LXXVII/7. - According to Andrijana Pravidur, this fibula is not in the inventory of grave N. Šokić II 2. 316 Grave M. Petrović Jr. 39: Truhelka 1904, 100 and Pl. L/9. Grave N. Šokić I 1: Truhelka 1904, 111 and Pl. LVIII/13. - Grave I. Stipančević 8: Truhelka 1904, 129 and Pl. LXXV/4, 11. - One of the fibulae from the last grave is identified by GAVRANOvić 2011a, 197 and Fig. 184/4 as the Potpećine type, but its other characteristics match the other fibula from the grave, which has been defined as the Borajna type.

317 Čović 1987a, 246, 250 and Pl. XXVII/2. - Gavranović 2011a, 197 and Fig. 184/1-2.

318 Čović 1987b, 618-619 and Pl. LXII/21-22, 30.

319 TERŽAN 1974, 41-45 and n. 47, Fig. 6. - TERŽAN 1987a, 17, 19 and Fig. 11. been proposed. In the graves, these fibulae usually appear in pairs, but they can also be single in combination with other types. Richer graves can even have several pairs of these fibulae. They are considered to be a characteristic form of the Glasinac IVc-2 phase; their appearance is dated to the first half of the $6^{\text {th }}$ century $\mathrm{BC}$ and partly continues into the $5^{\text {th }}$ century BC. They have been found in the greatest numbers at Glasinac and in southwest Serbia, where they were produced, but finds thereof have been recorded from northern Albania and Herzegovina to eastern Serbia, Syrmia, and Donja Dolina. ${ }^{320}$ We already indicated the contacts between Donja Dolina and the eastern Carpathian Basin on the basis of certain forms of belts and fibulae; in that respect, there is an interesting find of a small single-loop fibula with a triangular foot and rhombic cross section to the bow with ribbed ends that can be included in the Borajna type. The fibula comes from an unknown site in Banat ('Moldova Veche') and has been dated to the $6^{\text {th }}$ and $5^{\text {th }}$ centuries BC. ${ }^{321}$

Thus, small bronze single-loop fibulae of the Borajna type in the cemetery of Donja Dolina point to direct contacts with the area of Glasinac, which has an important role in the development of fibulae with a triangular or trapezoidal foot, where local and foreign elements are combined into original forms. These fibulae were a characteristic item of female costume, but also of male costume. In Donja Dolina they have been found in graves that can be dated to the late $7^{\text {th }}$ and the first half of the $6^{\text {th }}$ century BC. Their appearance in Donja Dolina, where they have been found in graves with forms that are characteristic for the southeastern Alps and the western Carpathian Basin (i.e. boat-shaped fibulae, ribbed-bow fibulae) allows for a correlation between the chronological phases in these areas. ${ }^{322}$

\subsection{Fibulae with a Ring at the Top of the Bow}

One of the distinctive forms of bronze fibulae that appears in the Donja Dolina cemetery with temple rings of types A and B (Tab. 4) in several graves consists of small single-loop bow fibulae with a ring at the top of the bow and a narrow and long triangular foot that can also have a ring at the end. The bow is usually smooth in the middle part where the ring is. The ring can have small hoops with hanging trapezoidal pendants (Figs. 4/6, 7/12). ${ }^{323}$ A fibula of this kind has

320 VAsIĆ 1977, 24 and Pls. 27/16; 30/1-2. - VAsIĆ 1999, $92-93$ and Pls. 45-47/691-772; for their distribution: Pl. 64B. - Within this separate type, there are fibulae of the Borajna and the Potpećine types after the classification of Teržan.

321 BADER 1983, 105 and Pl. 34/308.

322 TeržAn 1987a, 19.

323 Grave M. Petrović Jr. 6: Truhelka 1904, 91 and Pl. XLIII/13. Grave M. Petrović Sen. 4: Truhelka 1904, 122. - Grave M. Petrović Sen. 9: Truhelka 1904, 124 and Pl. LXXI/3. 
been found in another grave with another kind of temple ring. ${ }^{324}$ These fibulae are classified by Truhelka as the Velem-Szentvid-type fibulae and they are dated to phase $2 b,{ }^{325}$ or classified into the horizon with belt sets, Borajna-type fibulae, and dated to Ha D1. ${ }^{326}$ It has been noticed that these fibulae appear in the graves of richly equipped women from the beginning of the $6^{\text {th }}$ century BC and announce the Velem-Szentvid-type fibulae from the developed $6^{\text {th }}$ century BC. ${ }^{327}$ The younger fibulae of the Velem-Szentvid type, when compared to the mentioned ones from Donja Dolina, aside from some common characteristics such as the finely ribbed bow and the long triangular foot, have more rings at the top of the bow as a distinctive feature. The rings have small hoops with hanging pendants. The fibulae of this kind are distributed primarily at the sites in western Hungary, with the settlement and cemetery of Sopron-Krautacker as a prominent site, and dated to Ha D2-D3. ${ }^{328}$ Thus, fibulae with a ring at the top of the bow indicate contacts with the western Carpathian Basin.

\subsection{Torcs}

One of the distinctive items of ring jewellery in the cemetery, frequently found with temple rings of types A and B (Tab. 5), consists of torcs, which are most often found singly in a grave; however, there are graves with several of them. Depending on the shape of the body, there are several basic types, the most prolific being twisted torcs with rhombic cross-section terminals which are smooth or decorated with groups of oblique grooves and which are bent into the hole (Figs. 2/5, 3/2, 4/10, 5/1, 7/7). Only one torc from grave M. Petrović Sen. 6 has a twisted body and a hammered smooth part that is also bent into the hole at the end. They are most often made of iron, but there are bronze ones too. ${ }^{329}$

324 Grave N. Šokić II 5: Truhelka 1904, 134 and Pl. LXXVII/26. 325 Čović 1987a, 248 and Pl. XXVII/1.

326 Teržan 1974, 43 and Fig. 6. - With few parallels at sites in the western Carpathian Basin and the southeastern Alps.

327 Gavranović 2011a, 196.

328 Jerem 1981, 204-206 and Pls. I/1, 16; II/1. - Ferete 1986, 261 and Figs. 9/11; 10/1-3. - TeRžAn 1998, 520. - KovAČEvić 2007, 98100 and Figs. 2-3.

329 Grave N. Čegrlja 3: Truhelka 1904, 85 and Pl. XL/5. - Grave M. Petrović Jr. 3: Truhelka 1904, 90 and Pl. XLII/15. - Grave M. Petrović Jr. 6: Truhelka 1904, 91 and Pl. XLIII/12. - Grave 1 from the garden of N. Šokić: TruhelKa 1904, 84 and Fig. 65. - Grave S. Jakarić 14: TruhelKa 1904, 108. - Grave M. Petrović 2: TruhelKa 1904, 118. - Gavranović 2011b, 128 and Fig. 158/1. - Grave M. Petrović 3: Truhelka 1904, 119. - Čović 1987a, 246 and Pl. XXV/14. - Grave M. Petrović 8: Truhelka 1904, 120. - Marić 1964, Pl. X/14. - Grave M. Petrović Sen. 4: Truhelka 1904, 122 and Pl. LXVIII/13. - Grave M. Petrović Sen. 6: Truhelka 1904, 123 and Pl. LXIX/8, 13, 15. - Grave M. Petrović Sen. 9: Truhelka 1904, 124 and Pl. LXX/19. - Grave M. Petrović Sen. 10: Truhelka 1904, 125 and Pl. LXXII/6.
Twisted and knotted torcs have been found in the graves from the oldest burial phases of Donja Dolina, dated to Ha C1-C2. ${ }^{330}$ Twisted, knotted or smooth torcs are dated to phases $2 \mathrm{a}$ and $2 \mathrm{~b}$ by Čović, who points out that the torcs with knots and bronze beads on the body are somewhat older, while those with a twisted body have a longer duration, stretching into the $6^{\text {th }}$ century BC. ${ }^{331}$ Twisted torcs appear at Glasinac during phase IVa; it is believed that they are inherited from the previous phase IIIc. They no longer appear in phase IVb. ${ }^{332}$ Twisted torcs with bent terminals also appear in the older phase of the Urnfield Culture in the southern Carpathian Basin and persist into the younger phase; torcs with terminals of rhombic cross section do not have a narrow time frame. They persist in the Donja Dolina cemetery until the late $6^{\text {th }}$ century BC. ${ }^{333}$ Thus, the existence of twisted torcs with holes is recorded from the beginning of the Urnfield Culture, with the smooth part of rounded cross section documented since $\mathrm{Ha} \mathrm{B} .{ }^{334}$ A detailed classification of torcs was made by Vasić, who describes numerous finds from Donja Dolina, where almost all the torcs with a twisted body and a hole at the end belong to the variant characterized by a smooth part of round or rhombic cross section, which is characteristic for younger finds; the smooth part is sometimes decorated with oblique transverse grooves. The twisted torcs are dated to the Late Bronze Age until the beginning of the Early Iron Age, while they appear in Donja Dolina in the graves dated from the second half of the $7^{\text {th }}$ to the second half of the $6^{\text {th }}$ century BC..$^{335}$

The next group consists of torcs with the body segmented by beads. Grave M. Petrović Sen. 6 contained a small bronze torc with knots and a smooth upper part that is bent into the hole. ${ }^{336}$ One of the oldest graves, cremation grave S. Jakarić 14, also contained a bronze torc with beads; it seems that its smooth terminals were damaged and repaired by joining with rivets and bent into the hole (Fig. 8/1)..$^{337}$

330 TERŽAN 1974, 43 and Fig. 7.

331 Čović 1987a, 241-242, 246 and Fig. 15/10, Pl. XXIV/20.

332 Čović 1987b, 585 and Fig. 33/1. - Gavranović 2011a, 213.

333 Gavranović 2011a, 213-215 and Fig. 211. - Also, there is the appearance of a torc with thick grooves over the whole body from grave I. Stipančević 8 (GAvranović 2011a, Fig. 212/3), which appear starting from the younger Urnfield Culture. There are twisted torcs with smooth terminals with no curved ends in graves N. Šokić I 1 and I. Stipančević 16 (Gavranović 2011a, Fig. 213/2); the torc from the latter grave actually has broken terminals so it is not known how they ended. These latter date from the late $7^{\text {th }}$ and $6^{\text {th }}$ centuries BC.

334 MetZner-Nebelsick 2002, 445.

335 VAsić 2010, 34, 36, 38, 40-42 and Pls. 30-31. - Only the twisted torcs from grave M. Petrović Sen. 6 (TRuhelka 1904, 123 and Pl. LXIX/13) belong to the variant without a smooth part before the hole.

336 Truhelka 1904, 123 and Pl. LXIX/8.

337 Truhelka 1904, 108 and Pl. LVI/5. 
We should also mention the iron torc with broken terminals and strung bronze beads on the body, from grave M. Petrović $2,{ }^{338}$ dated to phase 2 a (Fig. 5/2)..$^{339}$ On the other hand, Gavranović dates both graves with knotted torcs to the horizon of the late $8^{\text {th }}$ and the early $7^{\text {th }}$ century BC, while, on the basis of the find of a fibula with the foot in the shape of a Boeotian shield, he dates the torc with bronze beads from grave M. Petrović 2 to the second half of the $7^{\text {th }}$ century BC and considers it a local form. ${ }^{340}$ Torcs with spherical expansions and terminals bent into the hole appear during $\mathrm{Ha} \mathrm{C}$ as a characteristic item of female jewellery, primarily in the southeastern Alpine area, the western Carpathian Basin, and Tyrol. ${ }^{341}$ In Dolenjska region, they reach the Stična phase. ${ }^{342}$ Carola Metzner-Nebelsick also considers them an eastern Alpine jewellery form of the Early Iron Age and concludes that the twisted torcs with tapering terminals display a Balkan influence. ${ }^{343}$

Thus, different forms of torcs from the Donja Dolina cemetery show that it is a prolonged, distinctive, and favourite form of female ring jewellery, which appears in the early phase of the Early Iron Age and remains in use until the second half of the $6^{\text {th }}$ century BC. Precisely because of the long duration of certain forms, e.g. torcs or bracelets with overlapping terminals, the Donja Dolina cemetery is important for the research of ring jewellery in the Early Iron Age.

\subsection{Bracelets with Stamp-Shaped Terminals}

One of the distinctive items of ring jewellery that appears together with temple rings of types A and B (Tab. 5) consists of massive bronze bracelets of round or oval cross section with expanding or stamp-shaped overlapping terminals that almost always come in pairs. A bracelet with gently expanding terminals has been found in grave S. Jakarić 14 (Fig. 8/9), ${ }^{344}$ while grave N. Šokić II 4 contained two bracelets of this shape (Fig. 9/1). ${ }^{345}$ On the other hand, grave N. Čegrlja 3 (Fig. 2/7) ${ }^{346}$ and grave M. Petrović Jr. 3 (Fig. 3/5) 347 each contained a pair of bracelets ending in

338 Truhelka 1904, 118. - Gavranović 2011b, 128 and Fig. 158/2. 339 Čović 1987a, 240-241.

340 Gavranović 2011a, 215-216 and Fig. 215.

341 TeržAn 1990, 99 and Map 11. - Vasić 2010, 44 and Pl. 34/225. Gavranović 2011a, 215. - EgG, Kramer 2016, 37-38 and Map 21.

342 Gabrovec 1987, 52 and Pl. IV/2, Fig. 3/14. - Dular 2003, 118 and Figs. 69B/12-13; 70A/1.

343 Metzner-Nebelsick 2002, 445-446 and Fig. 193. - According to Ć. Truhelka, the mentioned torcs have broken terminals.

344 Truhelka 1904, 108 and Pl. LV/2.

345 Truhelka 1904, 133 and Pl. LXXVII/10.

346 Truhelka 1904, 85 and Pl. XL/7.

347 Truhelka 1904, 90 and Pl. XLII/17-18. stamp-shaped expanding terminals and having the body decorated with groups of narrow transverse grooves. Pairs of such bracelets have also been found in graves N. Šokić I 1 and $8,{ }^{348}$ while one is a stray find. ${ }^{349}$

Massive bronze bracelets with overlapping terminals, like the one from grave S. Jakaric 14, appear in the Glasinac IVa phase and continue into the younger phases, when their different variants become the standard form of the bracelet. ${ }^{350}$ Bracelets with stamp-shaped terminals are dated to Ha C2 by Teržan, who believes that they originate from Glasinac, ${ }^{351}$ while Čović dates them to phases $2 \mathrm{a}$ and $2 \mathrm{~b} .{ }^{352}$ In the same way, they are dated to the contemporary horizon 2 by Vasić, who points out that they are often richly decorated by punching and incising. They are known from inhumation graves at Hrtkovci in Syrmia, from graves in Grivac, Novo Selo-Dub, and from destroyed graves at Vrtište. ${ }^{353}$ The bracelets from the inhumation grave at Hrtkovci have a transverse rib at the joint with the stampshaped terminals, while the body at the terminals is decorated with narrow transverse grooves that flank series of dots in the central part. ${ }^{354}$ There is also a find at Dvorovi near Bijeljina: a pair of decorated bracelets that do not expand at the overlapping terminals. ${ }^{355}$ At Glasinac, bronze bracelets with overlapping terminals, usually decorated with incised and punctured motifs, are an important form of phase IVb, when they come in several different variants, with the remark that they continue from the previous phase, IVa. They continue during phase IVc-1 too, when thicker bracelets appear along with the more gracious ones from the previous phase. Massive bracelets with overlapping terminals appear at that time too, preceding the very massive ones from the next phase, IVc-2, when they do not appear so frequently anymore; the heavy and massive bracelets are found in princely graves. ${ }^{356}$ Gavranović points out that the development of massive bronze bracelets of rounded cross section

348 Truhelka 1904, 111, 113 and Pls. LVIII/17, 19; LX/25.

349 Marić 1964, Pl. VI/17. - Gavranović 2011b, 80 and Fig. 102/3. 350 Čović 1987b, 583-584, 586 and Pl. LX/5, 11.

351 TeržAn 1974, 43 and Fig. 7.

352 Čović 1987a, 246.

353 VAsIĆ 1977, 18-19, 21, 33 and Pls. 21/17-18; 22A/3-4; 22B/2; 23/5-6; 26/22-23; 28/5-6. - Zотоvić 1985, 75 and Pl. XXII/12-13. VASIĆ 1987b, 544. - VAsIć 1987c, 655, 661 and Fig. 37/14, Pls. LXVIII/18-19; LXIX/4. - Kapuran, Blagojević, Bizjak 2015, Fig. 8/3. 354 Vinski, Vinski-Gasparini 1962, 270-271, 278 and Pl. VI/73-74. - Dizdar 2020, 193 and Fig. 3. - Also from Erdut, there is a fragment of a bracelet with stamp-shaped terminals and decorated body: Vinski, Vinski-GasParini 1962, 270 and Pl. IX/98.

355 Marić 1960, 49-50 and Pl. I/1-2.

356 Čović 1987b, 586, 611, 621 and Figs. 35/2-3; 36/15, Pl. LXI/15, 18. 
can be followed from the older phase of the Urnfield Culture into the $6^{\text {th }}$ century BC. The bracelets in the Jablanica cemetery have been dated to the $8^{\text {th }}$ century $\mathrm{BC}$ and continue into the $7^{\text {th }}$ century BC, while those from Donja Dolina have been dated to the late $8^{\text {th }}$ and the early $7^{\text {th }}$ centuries BC and represent a local form in terms of decoration. In the $7^{\text {th }}$ century BC, at Glasinac and in western Serbia, bracelets with stamp-shaped terminals appear along with the older form of bracelets. The youngest bracelets are the oversized ones, like the find from Arareva gromila, which continue into the second half of the $6^{\text {th }}$ century $\mathrm{BC}$ and are still decorated in the older way, with series of impressed dots and transverse grooves. ${ }^{357}$ A detailed classification of bronze bracelets was made by Gergova. Bracelets with gently expanding terminals, which may overlap, touch, or be slightly apart, have different body cross sections, and most of them are undecorated. Bracelets from graves S. Jakarić 14 and N. Šokić II 4 are most similar to those from Krăvenik and Debnevo, which range from the first decades of the $7^{\text {th }}$ to the middle of the $6^{\text {th }}$ century BC. Other bracelets from Donja Dolina have parallels in the bracelets with stamp-shaped terminals that overlap, rarely touching, and the terminals are usually decorated with groups of transverse lines. Having a round or $\mathrm{D}$ cross section, they are dated from the second half of the $7^{\text {th }}$ to the first half of the $6^{\text {th }}$ century BC. The last variant of the bracelet with stamp-shaped terminals are those that have conical expanded terminals which are separated from the body by a small rib. The overlapping terminals are decorated with transverse grooves, while the middle part is decorated with dots. This is probably the same kind of bracelet as those from grave N. Šokić I 8 and from Hrtkovci. They have also been dated to the second half of the $7^{\text {th }}$ and the first half of the $6^{\text {th }}$ century BC. ${ }^{358}$ Bracelets with stamp-shaped terminals, like open-work belts, are distributed from Macedonia to northern Serbia and northwest Bulgaria. ${ }^{359}$

Thus, massive bronze bracelets with overlapping stampshaped terminals, like open-work belts, indicate direct contacts of Donja Dolina with the areas of northern and eastern Serbia and even further to northwest Bulgaria. They are a characteristic item of female jewellery and have been found in graves that can be dated to the second half of the $7^{\text {th }}$ and the beginning of the $6^{\text {th }}$ century BC.

357 Gavranović 2011a, 210-211 and Figs. 206-207.

358 Gergova 1987, 70-73 and Pls. 33-35.-Hristov 2004, 92, 98 and Fig. 61.

359 Kilian 1973, 432. - Kilian 1975, 62, 108 and Fig. 4/1, Pl. 84/2. PARZINGER 1992, 234 and Fig. 12/2.

\subsection{Spiral Bracelets}

The distinctive ring jewellery in Donja Dolina that appears together with temple rings of types A and B, but not combined with bracelets with stamp-shaped terminals (Tab. 5), consists of bronze spiral bracelets that always come in pairs and are worn on the forearms. They have a large number of coils and are made of bronze wire of rectangular cross section. Such bracelets have been found in grave M. Petrović Jr. 6 (Fig. 4/8), ${ }^{360}$ grave 1 in the garden of N. Šokić, ${ }^{361}$ and grave M. Petrović Sen. 9 (Fig. 7/14), ${ }^{362}$ and there is also a chance find in the cemetery. ${ }^{363}$ Spiral bracelets consisting of four coils have been found in grave M. Petrović Sen. 6. ${ }^{364}$ Also mentioned were bracelets made with fewer coils found in grave M. Petrović 3. ${ }^{365}$

The spiral bracelets in the Donja Dolina cemetery are dated by Teržan to Ha D1, when they appear together with Borajna-type fibulae, fibulae with a ring at the top of the bow, and ribbed-bow fibulae, ${ }^{366}$ but they first appeared in female graves during the $8^{\text {th }}$ century BC. ${ }^{367}$ For Čović, this is a new form of bracelet, consisting of 17 to 20 coils, from phase $2 b .{ }^{368}$ At Glasinac, spiral bracelets with a small number of coils appear in phase IVa from the $8^{\text {th }}$ century BC, with the remark that they are also characteristic for the previous phase, IIIc, and disappear in phase IVb. On the other hand, spiral bracelets consisting of a large number of coils appear in phase IVb, when bracelets consisting of a narrow strip of bronze tin or wire are also found. It is a simple type of jewellery with a long duration, where those consisting of bronze wire of rounded cross section come in two variants during phase IV c-1: with a smaller or larger number of coils. Their duration is further documented during phase IVc-2. ${ }^{369}$ The spiral bracelets of bronze wire of round, triangular, or strip cross section are dated by Gavranović from the younger phase of the Urnfield Culture to the Late Hallstatt, representing a characteristic item of female jewellery over a long period, with the cross section being chronologically irrelevant. At the beginning of the Early Iron Age they had fewer coils (four to eight), while those with more coils from Donja Dolina have been dated by means of fibulae to the late

\footnotetext{
360 Truhelka 1904, 91 and Pl. XLIII/16-17.

361 Truhelka 1904, 84 and Fig. 65.

362 Truhelka 1904, 125 and Pl. LXXI/6-7.

363 Truhelka 1904, 146 and Fig. 92. - Marić 1964, Pl. X/7.

364 Truhelka 1904, 123 and Pl. LXIX/6-7.

365 Truhelka 1904, 119.

366 TERŽAN 1974, 43 and Fig. 7.

367 TeržAn 1987a, 12 and Figs. 5/6-7; 6/6-7.

368 Čović 1987a, 246, 250 and Fig. 15/6.

369 Čović 1987b, 582, 585, 598, 611, 621 and Pl. LX/7.
} 
$7^{\text {th }}$ and the early $6^{\text {th }}$ century BC. ${ }^{370}$ Their dating to horizon 2 is indicated by the pair of spiral bracelets found in the inhumation grave from Hrtkovci together with open-work belts and bracelets with stamp-shaped terminals; ${ }^{371}$ more rarely, their appearance has also been dated to horizon 3 on the basis of the finds from grave 6 of tumulus III in Pilatovići or from Kremna. ${ }^{372}$ In Bela Krajina there are spiral bracelets made of bronze and iron, dated to subphase Stična $1 .^{373}$

Thus, bronze spiral bracelets with a smaller or larger number of coils represent a characteristic item of female ring jewellery; in Donja Dolina, they have been found in graves that can be dated from the middle of the $7^{\text {th }}$ to the beginning of the $6^{\text {th }}$ century BC.

\subsection{Pendants}

Some characteristic forms of pendants were also found with temple rings of type A (Tab. 2). The richly furnished grave 3 from the Budești-Fînațe cemetery included three bronze pendants with an open-work cross motif in the middle, without a ring from which to be hung, drawing parallels with Donja Dolina. ${ }^{374}$ Also, grave 2 in the Teiuș-Cetățuia cemetery included a wheel-shaped pendant with four crossbars that get further apart towards the ring. ${ }^{375} \mathrm{~A}$ parallel for this type of wheel-shaped pendant with four crossbars is known from Glasinac, from the princely grave 1 of tumulus II at Ilijak, which seems to contain finds originating from several grave assemblages. Another pendant has been found at Gosinja planina, in tumulus XXXV, but outside the grave. ${ }^{376}$

Parallels for the described pendants have been found in inhumation grave M. Petrović Sen. 10, where there were two pendants on the chest of the woman: one open-work pendant with a double motif of the cross and four triangular parts with a crossbar in the middle, and one wheel-shaped

370 Gavranović 2011a, 207-208 and Figs. 200-202.

371 Vinski, Vinski-Gasparini 1962, 271, 278 and Pl. VI/72. - Vasić 1977, 18 and Pl. 22A/2. - DizDAR 2020, 193 and Fig. 3.

372 VAsić 1977, 24 and Pls. 27/19, 21; 30/7-8. - Zotović 1985, Pls. XXV/10-11; XXIII/7. - With fibulae of the Potpećine type: Vasić 1987b, 544. - Kapuran, Blagojević, Bizjak 2015, 67 and Fig. 8/3, Pl. 15/15.

373 Starè 1978, 68-69. - Dular 2003, 118 and Figs. 69B/14-17; 70A/3-4.

374 Marinescu 1984, 48 and Fig. 3/7. - Vulpe 1990, 98, 128 and Pl. 42B. - The grave also contained a bronze pendant with two bird protomes and six rings from which trapezoidal pendants hang. 375 Vasiliev 1970, 41-42 and Fig. 1/1. - Vasiliev, Badea, Man 1973, 28, 31 and Fig. 4/1. - VulPe 1990, Pl. 46C. - The grave also contained a circular pendant decorated with a hammered motif of concentric circles.

376 Benac, Čović 1957, 12, 23 and Pls. XX/13; XXXXVII/29. pendant with eight crossbars. ${ }^{377}$ Considering the find of a double-loop fibula with a ribbed bow and triangular foot, grave 10 has been dated to the oldest burial horizon in Donja Dolina. ${ }^{378}$ It can be mentioned that this grave from Donja Dolina also included a Kannenverschluss pendant. ${ }^{379}$ The pendant is believed to be an import from the Macedonian-Greek world of the first half of the $7^{\text {th }}$ century BC and belongs to type C, which is mostly distributed in Thessaly, the Peloponnese, and Greek Macedonia. ${ }^{380}$

As we already pointed out, the open-work pendants from the graves in Budești-Fînațe and Donja Dolina do not have a ring for hanging, which is considered to be an older typological characteristic. Their parallel is a much simpler pendant from tumulus III at the Cepari-Toplița site, where an adult was buried with potsherds and a bronze saltaleone. ${ }^{381}$ A pendant of this kind, only with a triangular ring at the top, originates from the Kisravazd hoard in western Hungary. Around the lower part of the pendant, there is a ring with two hammered trapezoidal pendants. Different variants of boat-shaped fibulae have been used to date the hoard to $\mathrm{Ha}$ C2/D1, or the end of the $7^{\text {th }}$ and the beginning of the $6^{\text {th }}$ century BC. ${ }^{382}$ A contemporary find is Vaskerestes - it is a hoard or destroyed grave -, where there was also an open-work pendant which also had a triangular ring at the top. ${ }^{383}$ Katalin Jankovits mentions the finds of wheelshaped pendants with V-shaped open-work decoration and a triangular or bow-shaped hanging ring from the sites in western Hungary, which she dates to $\mathrm{Ha}$ C. She also mentions the find of a pendant in the Celldömölk-Ság-Berg settlement from the younger phase of the Urnfield Culture. ${ }^{384}$ These decorative pendants were found by Teržan at sites in Italy from the late $8^{\text {th }}$ and the early $7^{\text {th }}$ century BC, dating the mentioned finds from western Hungary to the early Ha D. ${ }^{385}$ These pendants are also mentioned in the analysis of the Late Hallstatt princely grave under a tumulus from Rovná in southern Bohemia, with numerous prestigious

377 Truhelka 1904, 125 and Pl. LXXII/20-21. - Type D1a and D1b temple rings were found in the grave.

378 Teržan 1974, 43 and Fig. 7. - Gavranović 2011a, 233, 265. Gavranović 2016, 132 and Fig. 3 dates the grave to the early $7^{\text {th }}$ century BC.

379 Truhelka 1904, 125 and Pl. LXXII/12.

380 TeržAn 1974, 43. - Gavranović 2011a, 238. - For the pendant: Kilian 1975, 108, 114-115 and Pls. 29-30; 96/1. - Kilian-DirLmeIER 1979, 206-208 and Pl. 107.

381 Marinescu 1984, 79-80. - Popescu, Vulpe 1982, 87 and Fig. 4B/b.

382 Fenete 1973, 345, 350 and Pl. XLVI/30.

383 Mozsolics 1942, 160 and Pl. XV/26.

384 Jankovits 2017, 192-193 and Pls. 70/2500-2502; 111A.

385 TERŽAN 1990, 183. 
finds. The grave contained at least seven specimens of decorative spherical open-work bronze fittings, with the motifs of crosses and triangles, and a central hemispherical iron head, which are part of the decoration of a four-wheel wagon. In the Early Iron Age, similar spherical rings with an open-work cross decoration were a part of the cover of wagons distributed north and northwest of the Alps. ${ }^{386}$

\section{Conclusion}

The tradition of wearing bronze temple rings in the southern Carpathian Basin has been documented since the younger phase of the Urnfield Culture and continued during the Early Iron Age, with a noticeable change in forms, but also in the manner in which they were worn. As a result of the social and cultural reconfiguration in the observed area, which is clearly visible in the female costume and jewellery, and which started at the end of the first half of the $7^{\text {th }}$ century BC, new forms of temple rings appeared, which are now most clearly testified to by the finds from the Donja Dolina cemetery, with the documented contexts subjected to a complex archaeological analysis intended to define the basic forms and their chronological features. The questions focused on following the changes in the fashion of wearing head/hair jewellery, which followed the examination of the age profile of the wearers and their social position. On the other hand, the Dalj group cemeteries in the Danube area, especially during the youngest phases encompassing the initial phases of the Early Iron Age and its conclusion at the end of the $7^{\text {th }}$ and the beginning of the $6^{\text {th }}$ century BC, included numerous cremation graves with finds of small spiral bronze ringlets as the local hair decoration. In the graves, mostly of adult women, there were often several ringlets, and the manner of their wearing is indicated by the finds from inhumation grave 91 in Sotin, which show that they were probably worn on a band in the hair.

In the oldest burial phases in Donja Dolina, starting at the end of the first half of the $7^{\text {th }}$ century BC, in the graves of adult women furnished with costume or jewellery items completely different forms of temple rings are found. The finds of particular importance are those from inhumation graves - not only temple rings, but also other forms of costume and jewellery that enable the analyses of female bodily ornamentation indicating that they were gender- and often age-characteristic items. Certain distinct types would be characteristic only for the initial phase of the cemetery, while others would continue, with certain changes in form, until the end of the Early Iron Age. Along with the difference in form, there is also a clear difference in dimensions

386 ChytráčEK et al. 2018, 304, 306-307 and Figs. 8A-B, 9-13, 17. between the finds from Donja Dolina and the contemporary finds from the Dalj group cemeteries. Also, it must be pointed out that the youngest graves of the Dalj group with the finds of temple rings chronologically match the oldest graves from Donja Dolina.

In Donja Dolina, supplemented by the finds from other sites and based on the differences in the design of the terminals of temple rings, four basic types are distinguished, with two of them classified further into variants according to the way of shaping the body (Fig. 1). On this occasion, two forms of temple rings have been analysed: type $\mathrm{A}-$ the Ciumbrud type - with two variants, consisting of smooth temple rings with conical thickenings at the terminals, and type B - the Donja Dolina type - consisting of temple rings with flat or conical terminals decorated with transverse grooves. Temple rings of these two types appear only in the oldest burial horizons in Donja Dolina, which have been distinguished as phases $2 \mathrm{a}$ and $2 \mathrm{~b}$ after Čović, or as the first three phases in the classification of Teržan. ${ }^{387}$ Within the same graves, they are accompanied by some other forms (type C1, type D1 with variants, type D2), but they continue in even greater numbers in the younger burial phases, which will be the subject of a future analysis. Type B temple rings have not been found in any other sites except Donja Dolina, so it can be assumed that this was a local form of temple ring, which went out of use after three or four generations. Different insights were documented by examining type A temple rings, which have characteristic conical thickenings at the terminals. This is a frequent form in the graves in Donja Dolina (Tab. 1). Still, the highest number of finds of this form of temple rings has been recorded in the inhumation graves of the Ciumbrud group in Transylvania and in the neighbouring cemeteries in northeast Hungary (Fig. 16), where they appear in the oldest graves of the Vekerzug group (Tab. 3). Could it indicate selective acceptance of that particular form in Donja Dolina? Furthermore, there is a noticeable difference in the manner of wearing temple rings in Donja Dolina as opposed to the cemeteries of the Ciumbrud group. In fact, in Donja Dolina there are most often several type A temple rings on each side of the head (Fig. 2), often in combination with other forms (types B and $\mathrm{C} 1$ ), while the finds from the inhumation graves of the $\mathrm{Ci}$ umbrud group show that there was always a single temple ring on each side of the head. On the other hand, there is a documented similarity in their dimensions and the manner of wearing them, meaning that they were probably worn

387 TerŽAn 1974, 43-45 and Fig. 7. - Čović 1987a, 238-251. Gavranović 2011a, 263-276. - Gavranović 2011b, 128-139. GAvranović 2016, 130-133. 
hanging from a band of organic material on the head, or on a cap or headscarf, maybe in braided hair. This is clearly different from the manner of wearing ringlets in the cemeteries of the Dalj group, which were probably also worn on a band in the hair, but around the entire head. It is particularly interesting that type A temple rings appeared at the same time in very distant areas such as Donja Dolina and the Transylvanian sites, where the finds from Donja Dolina should probably be considered as a cultural transfer that appears because of the realized contacts; we should not completely rule out the possibility of individual mobility of women who could have brought such temple rings to Donja Dolina. Temple rings with conical terminals were then adopted and integrated into the dominant local visual expression without changing the basic form. Interestingly, to date no such temple rings have been found in the areas between Donja Dolina and Transylvania and northeast Hungary.

The analysis of the items of costume and jewellery from female graves in Donja Dolina where temple rings of types $\mathrm{A}$ and $\mathrm{B}$ have been found and which belong to the oldest burial phases (Tabs. 1,3) shows the complex network of contacts with the communities from both neighbouring and remote areas - from the western Balkans with Glasinac in the south, across the western Pannonian and southeastern Alpine areas in the west, to the northern areas of the central Balkans lying along the Danube in the east. Precisely in the initial phase of the Donja Dolina cemetery, many forms of female costume and jewellery are the same as those from the sites in northeastern Serbia, northwestern Bulgaria, and southwestern Romania, with noticeable differences in the structure of items of costume and jewellery, indicating that their choice was selective. These are open-work belts, double-loop fibulae with a triangular foot, fibulae of the Marvinci-Gogoșu type, particular double-loop fibulae with the foot in the shape of a Boeotian shield and a decorated bow, and massive bracelets with stamp-shaped terminals. These are frequent forms in Donja Dolina, probably indicating a strong eastern influence in the formative phase of the Donja Dolina cemetery or the existence of supra-regional female costume and jewellery from the middle of the $7^{\text {th }}$ century to the end of the first quarter of the $6^{\text {th }}$ century BC. We should certainly include the contemporary and distinct influences from Glasinac in the south, which can also be seen in specific items of female costume.

Thus, in the Donja Dolina cemetery, the same grave assemblages often combine a local form made in an original and innovative way (types B and C1) with a form (type A) that probably represents the influence of Transylvania and northeast Hungary. However, type A temple rings are still the only forms in the Donja Dolina cemetery that can have such an origin, while there are no known temple rings from the north Bulgarian sites, even though they share a common design characteristic - the existence of conical expansions at the terminals - while the form of the body is different. After their adoption, type A temple rings were probably also produced in Donja Dolina, similarly to other costume and jewellery items which originated elsewhere, but there was no change or adaptation in the original form. The cemetery in Donja Dolina is special because of the integration of different elements and their transformation into a characteristic outside expression that is currently best seen in the graves of women. It turns women into a distinct gender group, and their costumes and jewellery are the best evidence about a complex cultural landscape. Whatever the way in which the outside elements reached Donja Dolina - probably through cultural transfers and the individual mobility of women hybrid combinations of costume and jewellery appeared which became a visually distinctive code of the community, but also an important part of the visual identity of women. Moreover, the graves with variant A1 of temple rings in Donja Dolina have a structure of other finds that clearly differs from the graves of the Ciumbrud group, where there is a noticeable lower number of other items of costume and jewellery (Tab. 2). Inhumation graves of adult women have no belts, and fibulae are rare, just like other items of ring jewellery. Precisely in this part, the cemetery in Donja Dolina shows similarities with the sites in northeastern Serbia, northwestern Bulgaria, and southwestern Romania on the one hand, while certain forms of costume (i.e. belt sets with buckles and buttons) are clearly related to Glasinac on the other. Contacts with the west are primarily documented by different forms of boat-shaped fibulae, but they would become more intense in the future phases of Donja Dolina. Finally, what makes the Donja Dolina cemetery unique is the adoption of many forms from different areas, along with the creation of their own forms, which results in unique hybrid combinations of costume and jewellery that were probably affected by personal choice too. This is why the cemetery in Donja Dolina is also exceptionally important for the chronological studies of the Early Iron Age in the southern Carpathian Basin.

On the other hand, the grave assemblages from Donja Dolina testify about the social position of the buried women with the finds of temple rings in the oldest phases of the cemetery dated from the middle of the $7^{\text {th }}$ to the end of the first quarter of the $6^{\text {th }}$ century BC, i.e. it would encompass three to four generations. ${ }^{388}$ It is about richly decorated deceased

388 On the interesting idea that the richly furnished female graves in Donja Dolina are older than the men's graves equipped with weapons and dated to the late $7^{\text {th }}$ century BC: Gavranović 2016, 133, 139. 
adult women, rarely young, equipped with numerous items of costume and jewellery indicating the wealth and prosperity of the community, but also of specific families that could prepare such a last farewell to their deceased. This points to the participation of the local elite in various communication spheres, meaning that the upper social layer became a suitable transmitter of ideas, lifestyle, and even the objects themselves, like temple rings with conical terminals. Moreover, the wearing of several pairs of temple rings of types $A$ and $\mathrm{B}$, and bracelets with stamp-shaped terminals, may indicate their status in the family, meaning that they could have been married women, since temple rings are most often found in the graves of adult women in the Donja Dolina cemetery, but also in the Ciumbrud group cemeteries. This is confirmed by the results of anthropological analyses for particular inhumation graves of the Ciumbrud group. These graves also provide an idealized image of the deceased and show their social position, probably as wives and mothers, or as women of reproductive age. Child graves containing temple rings are rare, but sometimes they are richly furnished, as in the case of grave 3 from the Budești-Fînațe cemetery where a girl aged 9 to 10 was buried; her status was probably a reflection of the position of her family.

The analysis of temple rings of types $\mathrm{A}$ and $\mathrm{B}$ from the Donja Dolina cemetery, along with the data collected through the research on the contemporary cemeteries of the Ciumbrud group, has shown that their wearing is not only a category that could be socially defined, but that it also carries a certain gender and age meaning. Also, there are noticeable differences between the distinctive forms from Donja Dolina and all the other neighbouring areas that influenced other elements of costume and jewellery in many ways. We must particularly point out the temple rings with conical terminals which are unknown anywhere else, except for the Ciumbrud group and particular cemeteries of the Vekerzug group (Fig. 16). Considering this continuity of wearing temple rings in Donja Dolina during the Early Iron Age, when some new forms appeared while the old ones disappeared, the next analysis will focus precisely on such forms, which will testify about whether there was a change in the significance of temple rings for female bodily ornamentation, but also for the identity, social status, and the age of the deceased who used to wear them. ${ }^{389}$

389 This article was funded by the Croatian Science Foundation through a project (IP-06-2016-1749): Iron Age Female Identities in the Southern Carpathian Basin (FEMINE).

\section{References}

BADER 1983

T. BAder, Die Fibeln in Rumänien. Prähistorische Bronzefunde XIV/6, Munich 1983.

Benac, Čović 1957

A. Benac, B. Čović, Glasinac II: Željezno doba. Sarajevo 1957.

BENEDIKOVÁ 2007

L. BenedikovÁ, Die Hallstatt- und latènezeitlichen Siedlungen in der Nordostslowakei, Slovenská numizmatika 18, 2007, 69-110.

BENEDIKOVÁ 2017

L. BENEDIKovÁ, Kulturkontakte des slowakischen Teils der Westkarpaten während der Hallstattzeit. In: E. MiroššAYová, C. PARE, S. Stegmann-Rajtár (Eds.), Das nördliche Karpatenbecken in der Hallstattzeit: Wirtschaft, Handel und Kommunikation in früheisenzeitlichen Gesellschaften zwischen Ostalpen und Westpanonnien. Archaeolingua 38, Budapest 2017, 335-381.

Berciu, Comșa 1956

D. Berciu, E. ComșA, Săpăturile arheologice de la Balta Verde şi Gogoșu (1949 și 1950), Materiale și cercetări archeologice II, 1956, 252-489.

Blečić Kavur, Miličević-Capek 2011

M. Blečić Kavur, I. MiličEvić-CAPEK, O horizontu ratničkih grobova 5. stoljeća pr. Kr. na prostoru istočne obale Jadrana i njezina zaleđa: primjer novog nalaza iz Vranjeva sela kod Neuma, Prilozi Instituta za arheologiju u Zagrebu 28, 2011, 31-94.

Blečić Kavur, Pravidur 2012

M. Blečić Kavur, A. Pravidur, Ilirske kacige s područja Bosne i Hercegovine, Glasnik zemaljskog muzeja 53/2011, 2012, 35-136.

BoJČić, LožNJAK DizDar, HRŠAK 2018

Z. BojČIĆ, D. LožNJAK Dizdar, T. Hršak, Nove spoznaje o kronologiji groblja Batina-Sredno na početku starijega željeznog doba, Prilozi Instituta za arheologiju u Zagrebu 35, 2018, 159-192.

Bujor 1958

Z. Bujor, O geto-dakijskoji kulture v Murigiole, Dacia II, 1958, 125-141.

CHOchorowski 1985a

J. Chochorowski, Die Vekerzug-Kultur: Charakteristik der Funde. Acta Scientiarum Litterarumque DCCXXIV, Schedae archaeologicae Fasciculus XXXVI, Warszawa - Kraków 1985.

ChOchorowski $1985 \mathrm{~b}$

J. Chochorowski, Die Rolle der Vekerzug-Kultur(VK) im Rahmen der skythischen Einflüsse in Mitteleuropa, Prähistorische Zeitschrift 60, 1985, 204-271.

Chochorowski 1987

J. Chochorowski, Rola Sigynnóv Herodota w środowisku kulturowym wczesnej epoki želaza na nizinie węgierskiej, Przegląd Archeologiczny 34, 1987, 161-218.

Chochorowski 1998

J. Chochorowski, Die Vekerzug-Kultur und ihre östlichen Beziehungen. In: B. HänsEL, J. MACHNik (Eds.), Das Karpatenbecken und die osteuropäische Steppe. Prähistorische Archäologie in Südosteuropa 12, Munich 1998, 473-491.

Chochorowski 2014

J. Chochorowski, Scytowie a Europa Środkowa: historyczna interpretacja archeologicznej rzeczywistości, Materiały i Sprawozdania Rzeszowskiego Ośrodka Archeologicznego XXXV, 2014, 9-58. 
CHYTRÁČEK et al. 2018

M. Chytráček, O. Chyojka, M. Egg, J. John, R. Kyselý, J. Michálek, P. Stránská, Spähallstattzeitliches Fürstengrab von Rovná in Südböhmen: Symbolische Kunstform der Elite 6./5. Jahr. v. Chr., ihre Inspiration und Funktion. In: B. GedigA, A. Grossman, W. Piotrowski (Eds.), Inspiracje i funkcje sztuki pradziejowej i wczesnośredniowiecznej / Inspirationen und Funktion der ur- und frühgeschichtlichen Kunst. Biskupińskie Prace Archeologiczne 13, Biskupin - Wrocław 2018, 283-308.

\section{CRIȘAN 1964}

I. H. CRIȘAN, Morminte inedite din sec. III. î.e.n. în Transilvania, Acta Musei Napocensis I, 1964, 87-110.

\section{CRIŞAN 1965a}

I. H. CRișAN, Săpături și sondaje în Valea Mijlocie a Mureșului (Lechnița, Cristești, Războini-Cetate), Acta Musei Napocensis II, 1965, 39-76.

\section{CRISTAN 1965 b}

I. H. CRișAn, Once more about the Scythian problem in Transylvania, Dacia IX, 1965, 133-145.

\section{CRISAN 1974}

I. H. CRIȘAN, Siebenbürgen in der jüngeren Hallstattzeit (VI.-IV. Jh. v. u. Z.). In: Symposium zu Problemen der jüngeren Hallstattzeit in Mitteleuropa. Bratislava 1974, 101-124.

Čović 1961

B. Čović, Donja Dolina. Nécropole de l’Âge du Fer. Inventaria Archaeologica 3, Bonn 1961.

Čović 1987a

B. Čović, Grupa Donja Dolina-Sanski most. In: A. Benac (Ed.), Praistorija jugoslavenskih zemalja V: Željezno doba. Sarajevo 1987, 232-291.

Čović 1987 b

B. Čović, Glasinačka kultura. In: A. BenAc (Ed.), Praistorija jugoslavenskih zemalja V: Željezno doba. Sarajevo 1987, 575-642.

Dizdar 2018

M. DizDar, Reflections about some specific finds of female costume in the southern Carpathian Basin: Can we recognize female mobility in the Middle La Tène? In: S. Berecki, A. Rustoru, M. Egri (Eds.), Iron Age Connectivity in the Carpathian Basin. Bibliotheca Mvsei Marisiensis, Series Archaeologica XVI, Cluj-Napoca 2018, 15-38.

Dizdar 2019

M. Dizdar, New Late Hallstatt finds from the Vinkovci region (eastern Croatia): a contribution to the study of impacts from the Balkans to the southeastern Carpathian Basin. In: V. Filipović, A. Bulatović, A. Kapuran (Eds.), Papers in Honour of Rastko Vasić $80^{\text {th }}$ Birthday / Зборник радова у част 80 г. живота Растка Bacuћa. Belgrade 2019, 319-343.

\section{DizDAR 2020}

M. DizDAR, The Late Hallstatt connections between the southeastern Carpathian Basin and the western and central Balkans: the beautiful ladies from the south. In: M. Gavranović, D. Heilmann, A. Kapuran, M. Verčík (Eds.), Spheres of Interaction. Contacts and Relationships between the Balkans and Adjacent Regions in the Late Bronze / Iron Age $\left(13^{\text {th }}-5^{\text {th }}\right.$ centuries BCE). Perspectives on Balkan Archaeology 1, Rahden/Westf. 2020, 189-215.

Dular 2003

J. Dular, Halštatske nekropole Dolenjske. Opera Instituti Archaeologici Sloveniae 6, Ljubljana 2003.

DušEK 1964a

M. DušEk, Regiunile carpato-dunărene și sudul Slovaciei în etapa hallstattiana tîrzie, Arheologia Moldovei II-III, 1964, 273-298.
DušEK $1964 b$

M. DušEK, Waren Skythen in Mitteleuropa und Deutschland?, Praehistorische Zeitschrift XLII, 1964, 49-76.

DUŠEK 1966

M. DušEK, Thrakisches Gräberfeld der Hallstattzeit in Chotín. Archaeologica Slovaca Fontes VI, Bratislava 1966.

DUŠEK 1974

M. DušEK, Die Thraker im Karpatenbecken, Slovenská Archeológia XXII/2, 1974, 361-434.

EGG 1996

M. EGG, Das hallstattzeitliche Fürstengrab von Strettweg bei Judenburg in der Obersteiermark. Monographien des Römisch-Germanischen Zentralmuseums 37, Mainz a. R. 1996.

EGG, KRAMER 2016

M. EgG, D. Kramer, Die hallstattzeitlichen Fürstengräber von Kleinklein in der Steiermark: Die beiden Hartnermichelkogel und der Pommerkogel. Monographien des Römisch-Germanischen Zentralmuseums 125, Mainz a. R. 2016.

FEKETE 1973

M. Fekete, Der Depotfund von Kisravazd, Acta Archaeologica Academiae Scientiarum Hungaricae XXV, 1973, 341-358.

FEKETE 1986

M. Fenete, Früheisenzeitliche Fibelherstellung in Transdanubien: Beiträge zur Geschichte der Toreutik und des Handels. In: D.-W. R. Buck, B. Gramsch (Eds.), Siedlung, Wirtschaft und Gesellschaft während der jüngeren Bronze- und Hallstattzeit in Mitteleuropa. Veröffentlichungen des Museums für Ur- und Frühgeschichte Potsdam 20, Berlin 1986, 249-266.

FELCAN 2018

M. FELCAN, There is no need for a culture? Case studies of Early Iron Age settlements in Small Carpathians, Musaica Archaeologica 2, 2018, 51-72.

FERENCZI 1965

Ș. Ferenczi, Cimitirul "scitic” de la Ciumbrud (partea I), Acta Musei Napocensis II, 1965, 77-105.

FERENCZI 1966

Ș. Ferenczi, Cimitirul “scitic” de la Ciumbrud (partea a II-a), Acta Musei Napocensis III, 1966, 49-73.

FERENCZI 1969

Ș. Ferenczi, Cimitirul "scitic” de la Ciumbrud (partea IV), Acta Musei Napocensis VI, 1969, 47-65

Filipović 2015

V. Filipović, Triballi as conservative Paleobalkan tribe in the $6^{\text {th }}$ and $5^{\text {th }}$ century BC. In: M. GušTin, W. DAvid (Eds.), The Clash of Cultures? The Celts and the Macedonian World. Schriften des Kelten Römer Museums Manching 9, Manching 2015, 47-52.

GABROVEC 1970

S. Gabrovec, Dvozankaste ločne fibule, Godišnjak Centra za balkanološka ispitivanja VIII/6, 1970, 5-65.

GABROVEC 1987

S. Gabrovec, Dolenjska grupa. In: A. Benac (Ed.), Praistorija jugoslavenskih zemalja V: Željezno doba. Sarajevo 1987, 29-119.

GabRovec 2010

S. Gabrovec, Stiške gomile. In: S. Gabrovec, B. Teržan, Stična II/2: Gomile starejše železne dobe: Razprave. Katalogi in Monografije 38, Ljubljana 2010, 7-60.

Gavranović 2011a

M. Gavranović, Die Spätbronze- und Früheisenzeit in Bosnien: Teil I. Universitätsforschungen zur Prähistorischen Archäologie 195, Bonn 2011. 
Gavranović 2011b

M. Gavranović, Die Spätbronze- und Früheisenzeit in Bosnien: Teil II. Universitätsforschungen zur Prähistorischen Archäologie 195, Bonn 2011.

GAVRANOVIĆ 2016

M. Gavranović, Zwischen Glaube und Prestige: Mediterrane Importe in der westbalkanischen Früheisenzeit. In: B. GEdigA, A. Grossman, W. Piotrowski (Eds.), Europa w okresie od VIII wieku przed narodzeniem Chrystusa do I wieku naszej ery / Europa zwischen 8 Jhd. v. Chr. Geb. bis 1 Jhd. u. Zeit. Biskupińskie Prace Archeologiczne 11, Biskupin - Wrocław 2016, 123-146.

Gergova 1987

D. Gergova, Früh- und ältereisenzeitliche Fibeln in Bulgarien. Prähistorische Bronzefunde XIV/7, Munich 1987.

GLunz 1997

B. E. Glunz, Studien zu den Fibeln aus dem Gräberfeld von Hallstatt, Oberösterreich. Linzer Archäologische Forschungen 25, Linz 1997.

GRAHEK 2004

L. Graher, Halštatska gomila na Hribu v Metliki, Arheološki vestnik 55, 2004, 111-206.

Heilmann 2016

D. Heilmann, Contextualising bow fibulae with Boeotian shield plates: cultural transfer processes during Early Iron Age in the central Balkan area, Старинар / Starinar LXVI, 2016, 9-26.

HOREDT 1953

K. Horedt, Cercetările arheologice din regiunea Hoghiz-Ugra şi Teiuş, Materiale Arheologice privind Istoria Veche a R.P.R. I, 1953, 785-815.

Hristov 2004

I. Hristov, Тракийски накитни съкровища (IX-VI в. пр. Xp.) от фонда на Националния исторически музей, Известия на Национамен Исторически Музей 14, 2004, 43-67.

ILON 2017

G. ILON, Skythische Spiralringe oder Piercings aus West-Transdanubien und ihre Symbolik. In: N. Beljak Pažinová, Z. Borzová (Eds.), Sedem decénií Petra Ramsauera. Studia Historica Nitriensia 21/Supplementum, Nitra 2017, 97-116.

IsLAMI 1983

S. IsLAmI, Aspects de la civilisation tumulaire de Mat dans la protohistoire, Studia Albanica XX/1, 1983, 99-126.

JANKOVITS 2017

K. Jankovits, Die bronzezeitlichen Anhänger in Ungarn. Studia ad Archaeologiam Pazmaniensia 9, Budapest 2017.

JAŠAREVIĆ 2014

A. JAŠAREvić, Socio-ekonomska i simbolička uloga importovanih metalnih posuda s Glasinca, Godišnjak Centra za balkanološka ispitivanja 43, 2014, 51-99.

JAŠAREVIĆ 2017

A. JašAREvić, Zaboravljeni grobovi iz Donje Doline, Glasnik Zemaljskog Muzeja 54, 2017, 7-30.

JEREM 1981

E. Jerem, Südliche Beziehungen einiger hallstattzeitlicher Fundtypen Transdanubiens, Materijali Saveza arheoloških društava Jugoslavije XIX, Novi Sad 1981, 201-220.

Kapuran, Blagojević, Bizjak 2015

A. Kapuran, M. Blagojević, D. BizjaK, Settlements and necropolises of the Early Iron Age along the middle course of the Nišava river, Старинар / Starinar LXV, 2015, 145-182.

\section{KemenCZei 1988}

T. Kemenczei, $\mathrm{Zu}$ den Beziehungen zwischen dem ungarischen Donau-Theißraum und dem NW-Balkan in der Früheisenzeit, Folia Archaeologica XXXIX, 1988, 93-113.

KemenCZei 1994

T. Kemenczei, Pfeilspitzen von Früh-Skythentyp aus Ostungarn, Folia Archaeologica XLIII, 1994, 79-99.

KemencZei 2002

T. Kemenczei, Beiträge zur Schmuckmode der Alföld-Gruppe skythischer Prägung, Folia Archaeologica XLIX-L/2001-2002, 2002, 29-77.

KemencZei 2004

T. Kemenczei, Bemerkungen zu den Fibeln des Skythenzeit, Communicationes Archaeologicae Hungariae 2004, 2004, 79-103.

KeMENCZEi 2009

T. Kemenczei, Studien zu den Denkmälern skythisch geprägter Alföld Gruppe. Inventaria Praehistorica Hungariae XII, Budapest 2009.

KeMENCZei 2010

T. Kemenczei, Funde der skythischen geprägten Alföld-Gruppe in Transdanubien, Folia Archaeologica LIV/2008-2010, 2010, 101-125.

KILIAN 1973

K. Kilian, Zur eisenzeitlichen Transhumanz in Nordgriechenland, Archäologisches Korrespondenzblatt 3, 1973, 431-435.

KiLian 1975

K. Kilıan, Trachtzubehör der Eisenzeit zwischen Ägäis und Adria, Praehistorische Zeitschrift 50, 1975, 9-140.

Kilian-Dirlmeier 1979

I. Kilian-Dirlmeier, Anhänger in Griechenland von der mykenischen bis zur spätgeometrischen Zeit. Prähistorische Bronzefunde XI/2, Munich 1979.

KovaČEvić 2007

S. KovačEvić, Karakteristični nalazi kasnohalštatskog naselja u Zbelavi kod Varaždina i fibula tipa Velem, Prilozi Instituta za arheologiju u Zagrebu 24, 2007, 89-112.

Kozubová 2013

A. Kozubová, Pohrebiská vekerzugskej kultúry v Chotíne na juhozápadnom Slovensku: Vyhodnotenie. Dissertationes Archaeologicae Bratislavenses 1, Bratislava 2013.

Kozubová 2018

A. Kozubová, Kopfschmuck der Vekerzug-Kultur am Beispiel der Gräberfelder in Chotín, Musaica Archaeologica 3/1, 2018, 13-63.

Kozubová 2019

A. Kozubová, "Something happened in the east but more in the west and south.": Einige kritische Bemerkungen zu östlichen Einflüssen in der Vekerzug-Kultur, Musaica Archaeologica 1/2019, 2019, 55-185.

KRUH 2010

A. Kruh, Gomila 5 ali Tratarjeva gomila. In: S. Gabrovec, B. TerŽAN, Stična II/2: Gomile starejše železne dobe: Razprave. Katalogi in Monografije 38, Ljubljana 2010, 69-131.

LOŽNJAK DiZDAR 2010

D. LožNJAK Dizdar, Grobovi uglednica mlađe faze kulture polja sa žarama u sjevernoj Hrvatskoj. Prilog poznavanju ženske nošnje kasnog brončanog doba na prostoru južne Panonije, Vjesnik Arheološkog muzeja u Zagrebu XLII/2009, 2010, 157-182.

LožNJAK Dizdar, Hutinec 2013

D. Ložnjak Dizdar, M. Hutinec, Sotin: arheološka istraživanja 2012. godine, Annales Instituti Archaeologici IX, 2013, 8-11. 
LožNJAK Dizdar, Rajić ŠIKANJIĆ 2016

D. Ložnjak Dizdar, P. Rajić Šikanjić, Funerary practices at the end of the Late Bronze Age in the southern Middle Danube region. In: V. Sîrbu, M. Jevtić, K. Dmitrović, M. Ljuština (Eds.), Funerary Practices during the Bronze and Iron Ages in Central and Southeast Europe. Proceedings of the $14^{\text {th }}$ International Colloquium of Funerary Archaeology in Čačak, Serbia, $24^{\text {th }}-27^{\text {th }}$ September 2015. Belgrade - Čačak 2016, 109-126.

LuCENTINI 1981

N. Lucentini, Sulla cronologia delle necropoli di Glasinac nell'età del ferro. In: R. Peroni (Ed.), Studi di protoistoria Adriatica 1. Quaderni di cultura materiale 2, Rome 1981, 67-171.

MARIĆ 1960

Z. MARIĆ, Praistorijskki nalazi i lokaliteti iz Triješnice i Dvorova kod Bijeljine, Članci i građa za kulturnu istoriju istočne Bosne IV, $1960,43-67$.

MARIĆ 1964

Z. Marić, Donja Dolina, Glasnik Zemaljskog Muzeja XIX, 1964, 5-128.

\section{Marinescu 1984}

G. MarinEscu, Die jüngere Hallstattzeit in Nordostsiebenbürgen, Dacia XXVIII/1-2, 1984, 47-83.

Medović, Medović 2011

P. Medović, I. Medović, Gradina na Bosutu: Naselje starijeg gvozdenog doba. Novi Sad 2011.

MetZner-Nebelsick 2002

C. MetZner-Nebelsick, Der “Thrako-Kimmerische” Formenkreis aus der Sicht der Urnenfelder- und Hallstattzeit im südöstlichen Pannonien. Vorgeschichtliche Forschungen 23, Rahden/Westf. 2002.

Mozsolics 1942

A. Mozsolics, A magyarkeresztesi (Vas megye) bronzlelet, Archaeologiai Értesítő III, 1942, 155-161.

NÉMETI 1982

I. Nemetı, Das späthallstattzeitliche Gräberfeld von Sanislău, Dacia XXVI/1-2, 1982, 115-144.

NicULIȚĂ et al. 2011

I. Niculiță, A. Zanoci, S. Matveev, M. Băț, Piese de port, podoabă și de toaletă din aşezarea traco-getică Saharna Mare. In: D. Măgureanu, D. Măndescu, S. Matei (Eds.), Archaeology: Making of and Practice. Studies in Honor of Mircea Babeș at his 70 Anniversary. Pitești 2011, 193-204.

PABst 2012

S. PAвST, Die Brillenfibeln: Untersuchungen zu spätbronze- und ältereisenzeitlichen Frauentrachten zwischen Ostsee und Mittelmeer. Marburger Studien zur Vor- und Frühgeschichte 25, Rahden/Westf. 2012.

PÁRDUCZ 1954

M. PÁRducz, Le cimetière hallstattien de Szentes-Vekerzug II, Acta Archaeologica Academiae Scientiarum Hungaricae IV, 1954, 25-91.

PÁRdUCZ 1955

M. PÁrducz, Le cimetière hallstattien de Szentes-Vekerzug III, Acta Archaeologica Academiae Scientiarum Hungaricae VI, 1955, $1-22$.

PÁRDUCZ 1965

M. PÁrducz, Graves from the Scythian Age at Ártánd (County Hajdu-Bihar), Acta Archaeologica Academiae Scientiarum Hungaricae XVII, 1965, 137-231.
PÁRduCZ 1966

M. Párducz, The Scythian Age cemetery at Tápiószele, Acta Archaeologica Academiae Scientiarum Hungaricae XVIII, 1966, 35-91.

PARZINGer 1992

H. Parzinger, Archäologisches zur Frage der Illyrier, Bericht der Römisch-Germanischen Kommission 72/1991, 1992, 205-261.

Parzinger 1995

H. Parzinger, Archäologische Beiträge. In: H. Parzinger, J. Nekvasil, F. E. Barth, Die Býčí skála-Höhle. Ein hallstattzeitlicher Höhlenopferplatz in Mähren. Römisch-Germanische Forschungen 54, Mainz a. R. 1995, 16-92.

Patay 1955

P. PatAy, Szkíta leletek a nógrádi dombvidéken, Folia Archaeologica VII, 1955, 61-74.

Patay 1961

P. PatAy, Az Alsótelekesi vaskori temető, Folia Archaeologica XIII, 1961, 27-50.

Patay, Kiss 2002

P. Patay, Z. Kiss, Az Alsótelekes-Dolinkai szkítakori temetó közöletlen síraji (az 1962. és 1964. évi feltárás erdményei), Folia Archaeologica XLIX-L/2001-2002, 2002, 79-141.

Pavlovič 2018

D. Pavlovič, Gospa z uhani z Grofovih njiv pri Drnovem. In: M. ČrešNAR, M. VINAZZa (Eds.), Srečanja in vplivi v raziskovanju bronaste in železne dobe na Slovenskem. Ljubljana 2018, 353-365.

Popescu, Vulpe 1982

E. Popescu, A. Vulpe, Nouvelles découvertes du type Ferigile, Dacia XXVI/1-2, 1982, 77-114.

Popović 1984

P. Popović, Кожица I и II: праисторијска насеља, Старинар / Starinar XXXIII-XXXIV/1982-1983, 1984, 135-136.

Popović, Vukmanović 1998

P. Popović, M. Vukmanović, Vajuga-Pesak: nekropola starijeg gvozdenog doba. Đerdapske sveske 3, Belgrade 1998.

Potrebica 2003

H. Potrebica, Požeška kotlina i Donja Dolina u komunikacijskoj mreži starijeg željeznog doba, Opuscula Archaeologica 27, 2003, 217-242.

PRENDi 1976

F. PRENDI, Un aperçu sur la civilisation de la première période du Fer en Albanie. In: M. Suić (Ed.), Jadranska obala u protohistoriji: Kulturni i etnički problemi. Zagreb 1976, 155-175.

Rustoiv 1996

A. Rustoiu, Metalurgia bronzului la daci (sec. II î. Chr. - sec. I d. Chr.): Tehnici, ateliere și produse de bronz. Bibliotheca Thracologica XV, Bucharest 1996.

Rustoiv 2002

A. Rustoiv, Războinici și Artizani de Prestigiu în Dacia Preromană, Interferente Etnice şi Culturale în Milenile I a.Chr. - I p.Chr. Vol. III. Cluj-Napoca 2002.

SPÂNU 2012

D. SPÂNU, Tezaurele dacice : Creația în metale prețioase din Dacia preromană. Bucharest 2012.

STARÈ 1978

V. STARÈ, Fibule z oblogo iz gomil pod Vinjim vrhom, Arheološki vestnik XXIX, 1978, 64-80. 
Stojić, Jacanović 2008

M. StojIć, D. Jacanović, Пожаревац, културна стартиграфија праисторијских мокалитета у Барничеву. Belgrade - Požarevac 2008.

TASIĆ 1969

N. TAsić, Кожице: Насење бронзаног доба. In: Ђ. Бошковић (Ed.), Старе културе у Ђердапу. Belgrade 1969, 38-39.

Tecco Hvala 2007

S. Tecco Hvala, Women from Magdalenska gora. In: M. Blečić, M. Črešnar, B. Hänsel, A. Hellmuth, E. Kaiser, C. Metzner-Nebelsick (Eds.), Scripta Praehistorica in honorem Biba Teržan. Situla 44, Ljubljana 2007, 477-490.

Tecco-Hvala 2012

S. Tecco Hvala, Magdalenska gora: Družbena struktura in grobni rituali železnodobne skupnosti. Opera Instituti Archaeologici Sloveniae 26, Ljubljana 2012.

TERŽAN 1974

B. TERŽAn, Halštatske gomile iz Brusnic na Dolenjskem. In: M. GušTIN (Ed.), Varia Archaeologica 1, Brežice 1974, 31-66.

TERŽAN 1987a

B. TERžAn, The Early Iron Age chronology of the Central Balkans, Archaeologia Iugoslavica 24, 1987, 7-27.

TERŽAN 1987b

B. TeržAn, Rezension: Claus Dobiat, Das hallstattzeitliche Gräberfeld von Kleinklein und seine Keramik. Schild von Steier, Beiheft 1, Graz 1980, Arheološki vestnik 38, 1987, 413-433.

TERŽAN 1990

B. TERŽAn, Starejša železna doba na Slovenskem Štajerskem. Katalogi in Monografije 25, Ljubljana 1990.

TeržAn 1995

B. TeržAn, Handel und soziale Oberschichten im früheisenzeitlichen Südosteuropa. In: B. HäNSEL (Ed.), Handel, Tausch und Verkehr im bronze- und früheisenzeitlichen Südosteuropa. Prähistorische Archäologie in Südosteuropa 11, Munich - Berlin 1995, 81-159.

TERŽAN 1998

B. TERžAn, Auswirkungen des skythisch geprägten Kulturkreises auf die hallstattzeitlichen Kulturgruppen Pannoniens und des Ostalpenraumes. In: B. HäNSEL, J. MACHNIK (Eds.), Das Karpatenbecken und die osteuropäische Steppe. Prähistorische Archäologie in Südosteuropa 12, Munich 1998, 511-560.

TERŽAN 2016

B. TeržAn, Bogenfibeln vom Typ Poiana und verwandte Formen zwischen dem Ostbalkan- und dem Ostalpenraum während der Hallstattzeit. In: A. Zanoci, E. KaIser, M. Kashuba, E. IzbiTSER, M. BĂȚ (Eds.), Mensch, Kultur und Gesellschaft von der Kupferzeit bis zur frühen Eisenzeit im nördlichen Eurasien. Beiträge zu Ehren zum 60. Geburstag von Eugen Sava. Tyrgetia International I, Chişinău 2016, 337-346.

Teržan, Hellmuth, Heimann 2011

B. Teržan, A. Hellmuth, F. Heimann, Amazonenmythos im Spiegel der eisenzeitlichen Grabfunde zwischen Pontus und Karpatenbecken. In: E. Sava, B. Govedarica, B. Hänsel (Eds.), Der Schwarzmeerraum vom Äneolithikum bis in die Früheisenzeit (5000-500 v. Chr.) 2: Globale Entwicklung versus Lokalgeschehen. Prähistorische Archäologie in Südosteuropa 27, Rahden/ Westf. 2011, 253-272.

Tessmann 2004

B. Tessmann, Grabhügel 30 aus Rusanovići: Untersuchungen zu Kontakten zwischen der Glasinac-Hochebene und dem westlichen japodischen Raum (Lika-Hochebene), Godišnjak Centra za balkanološka ispitivanja 31, 2004, 139-183.

То́тн 2012

F. M. Tóтн, Korai szkítakori sírok Dédestapolcsány-Verbece-Tetó lelőhelyen, A Herman Ottó Múzeum Évkönyve LI, 2012, 63-91.

Truhelka 1904

C. Truhelka, Der vorgeschichtliche Pfahlbau im Savebette bei Donja Dolina (Bezirk Bosnisch-Gradiška): Bericht über die Ausgrabungen bis 1904, Wissenschaftliche Mitteilungen aus Bosnien und der Herzegowina IX, 1904, 1-156.

URSUȚiU, URÁK 2016

A. Ursuțiu, M. Urák, Iron Age girl in a Bronze Age pit? A child burial of the Ciumbrud cultural group from Orosia (Hu: $\mathrm{Ma}-$ rosoroszu), Mureş County. In: F. Gogâltan, S. C. AilincăI (Eds.), Settlements of Life and Death: Studies from Prehistory to Middle Ages. Biblioteca Istro-Pontica, Seria Archeologie 14, Cluj-Napoca 2016, 255-272.

VASIĆ 1971

R. VAsIć, The openwork belts and the Early Iron Age chronology in the northern Balkans, Archaeologia Iugoslavica XII, 1971, 1-13.

VASIĆ 1974

R. VASIĆ, Нека питања хронологије старијег гвозденог доба северног Балкана, Старинар / Starinar XXII/1971, 1974, 35-51.

VASIĆ 1975

R. VAsIć, Donja Dolina i Makedonija, Godišnjak Centra za balkanološka ispitivanja XIV/12, 1975, 81-94.

VAsIĆ 1977

R. VAsIć, The Chronology of the Early Iron Age in Serbia. British Archaeological Reports Supplementry Series 31, Oxford 1977.

VAsIĆ 1982

R. VASIĆ, Prilog proučavanju grčkog oružja u Jugoslaviji, Godišnjak Centra za balkanološka ispitivanja XX/18, 1982, 5-24.

VASIĆ 1987a

R. VAsić, Prilog proučavanju lučnih fibula sa pravougaonom nogom na Balkanu, Arheološki vestnik 38, 1987, 41-68.

VASIĆ 1987 b

R. VAsić, Bosutska grupa. In: A. Benac (Ed.), Praistorija jugoslavenskih zemalja V: Željezno doba. Sarajevo 1987, 536-554.

VAsIĆ 1987 c

R. VAsić, Moravsko-timočka oblast. In: A. Benac (Ed.), Praistorija jugoslavenskih zemalja V: Željezno doba. Sarajevo 1987, 651672.

VASIĆ 1988

R. VASIĆ, Нове белешке о старијем гвозденом добу у Србији, Старинар / Starinar XXXIX, 1988, 1-13.

VASIĆ 1991

R. VAsić, Boat-shaped earrings in the central Balkan area, Старинар / Starinar XL-XLI/1989-1990, 1991, 135-140.

VASIĆ 1996

R. VAsIĆ, Quelques considérations sur le terme "Basarabi”. In: P. Roman (Ed.), Der Basarabi-Komplex in Mittel- und Südosteuropa, Archäologische Abteilung I, Bucharest 1996, 15-21.

VASIĆ 1999

R. VAsIĆ, Die Fibeln im Zentralbalkan. Prähistorische Bronzefunde IV/12, Stuttgart 1999.

VASIĆ 2010

R. VAsić, Die Halsringe im Zentralbalkan (Vojvodina, Serbien, Kosovo und Mazedonien). Prähistorische Bronzefunde XI/7, Stuttgart 2010. 
VAsiliev 1970

V. VAsiliev, Podoabe de metal prețios din morminte scitice în Transilvania, Acta Musei Napocensis VII, 1970, 41-63.

VASILIEV 1972

V. VAsiliev, Necropola sciticăde la Blaj: Legăturile inventarului şi ritului funerar cu necropolele scitice de la nordul Mării Negre, Apvlvm X, 1972, 19-64.

VASILIEV 1980

V. Vasiliev, Sciții Agatîrși pe teritoriul României. Cluj-Napoca 1980.

Vasiliev, Badea, Man 1973

V. VAsiliev, A. BADEA, I. Man, Două noi morminte scitice descoperite la Teiuş, Sargetia X, 1973, 27-43.

VASILIEV, ZRÍNYI 1974

V. VAsiliev, A. ZrínYi, Necropola scitică de la Ozd, File de Istorie III, 1974, 89-137.

VINSKI 1955

Z. VINSKI, “Tračko-kimerijski” nalaz u Adaševcu u Sremu, Rad vojvođanskih muzeja 4, 1955, 27-42.

VINSKI, VINSKI-GASPARINI 1962

Z. Vinski, K. VINSKI-GAsPARINI, O utjecajima istočno-alpske halštatske kulture i balkanske ilirske kulture na slavonsko-sremsko Podunavlje, Arheološki radovi i rasprave II, 1962, 263-293.

VINSKI-GASPARINI 1987

K. Vinski-Gasparini, Grupa Martijanec-Kaptol. In: A. BenaC (Ed.), Praistorija jugoslavenskih zemalja V: Željezno doba. Sarajevo $1987,182-231$.

VLASSA 1961

N. Vlassa, O contribuție la problema epocii scitice în Transilvania: Cimitirul de la Cipău-“Gară”, Apvlvm IIII, 1961, 19-49.

VulPE 1967

A. Vulpe, Necropola Hallstattiana de la Ferigile. Biblioteca de Arheologie XI, Bucharest 1967.

VulPE 1970

A. Vulpe, Archäologische Forschungen und historische Betrachtungen über das 7. bis 5. Jh. im Donau-Karpatenraum, Memoria Antiquitatis II, 1970, 115-183.

VulPE 1977

A. Vulpe, Zur Chronologie der Ferigile-Gruppe, Dacia XXI, 1977, 81-111.

VulPE 1984

A. Vulpe, Descoperirile hallstattiene din zona Aiudului, Thraco-Dacica V/1-2, 1984, 36-63.

Vulpe 1990

A. Vulpe, Die Kurzschwerter, Dolche und Streitmesser der Hallstattzeit in Rumänien. Prähistorische Bronzefunde VI/9, Munich 1990.

Zotović 1985

M. Zотоvić, Arheološki i etnički problemi bronzanog i gvozdenog doba zapadne Srbije. Dissertationes et Monographiae XXVI, Belgrade 1985.

ZRÍNYI 1965

A. ZRíNYI, Ĭnemnări arheologice pre marginea a două morminte găsite în cimitirul scitic din Cristești, Studii şi Materiale I, 1965, 27-50.
Marko Dizdar
Institute of Archaeology

Jurjerska ulica 32

10000 Zagreb

Croatia

marko.dizdar@iarb.br

orcid.org/0000-0003-3964-9002

Aleksandar Kapuran

Archaeological Institute

Kneza Mibaila 35/IV 11000 Belgrade

Serbia

a.kapuran@gmail.com 